\title{
Heat stress tolerance responses in developing tomato anthers
}

Craita Elena Bita 


\section{Thesis committee}

\section{Promotor}

Prof. Dr G.C. Angenent

Personal chair at the Laboratory of Molecular Biology

Wageningen University

\section{Co-promotor}

Dr Christian Bachem

Assistant professor, Laboratory of Plant Breeding

Wageningen University

\section{Other members}

Prof. Dr H.J. Bouwmeester, Wageningen University

Dr M. van Zanten, Utrecht University

Dr R.A. de Maagd, Wageningen UR

Dr M.J.M. Smulders, Wageningen UR

This research was conducted under the auspices of the Graduate School for Experimental Plant Science (EPS). 


\title{
Heat stress tolerance responses in developing tomato anthers
}

\author{
Craita Elena Bita
}

\section{Thesis}

submitted in fulfilment of the requirements for the degree of doctor

at Wageningen University

by the authority of the Rector Magnificus

Prof. Dr A.P.J. Mol

in the presence of the

Thesis Committee appointed by the Academic Board

to be defended in public

on Tuesday June $7^{\text {th }}, 2016$

at 1.30 p.m. in the Aula. 


\section{Craita Elena Bita}

Heat stress tolerance responses in developing tomato anthers

118 pages

$\mathrm{PhD}$ thesis, Wageningen University, Wageningen, NL (2016)

With references, with summaries in English and Dutch

ISBN: 978-94-6257-770-1

DOI: http://dx.doi.org/10.18174/377692 
This work was supported by the Marie Curie Research Fellowship Programme (ADONIS grant 2000327 to C.E.B) and additionally by a grant [personal, to C.E.B] from the Institute of Water and Wetland Research and the Radboud University Nijmegen (IWWR) in The Netherlands 


\section{Contents}

Chapter 1: General introduction

Chapter 2: Plant tolerance to high temperature and production of heat stress tolerant crops

Chapter 3: Temporal and spatial effects of moderate heat stress on gene expression in meiotic tomato anthers

Chapter 4: Anther transcriptome profiling of tolerant and sensitive tomato genotypes under moderate heat stress

Chapter 5: Heat stress, male sterility and ABA: a working hypothesis

Chapter 6: Concluding remarks

Summary English

Summary Dutch

Acknowledgements

Curriculum vitae

Education statement 


\section{Chapter 1}

General Introduction 


\section{Tomato as model crop in the study of heat stress effects on fruit set}

Tomato (Lycopersicon esculentum) is the second most important vegetable crop in the world, next to potato. Tomato production has gradually increased in the last decade despite the production area remaining almost the same and at present the global production is about 200 million tons fresh fruit produced on 3.7 million hectares. The top leading fruit-producing countries are Mexico, Brazil, Spain and Italy (FAOSTAT 2014) while the two leading countries in fruit yield per hectare are Belgium $(4,996,000 \mathrm{Hg} / \mathrm{ha})$ and the Netherlands $(4,835,973 \mathrm{Hg} / \mathrm{ha})$ (FAOSTAT Database, 2013). Tomatoes are consumed fresh or processed (sauce, paste, juice) and the demand for tomato products has increased substantially to a consumption rate of $72,7 \mathrm{~kg}$ per capita per year (Benton Jones, 2008). Field grown tomatoes are commonly produced in tropical, subtropical and warm temperate climates with a sufficiently long growing season for fruiting and fruit maturation, such as China, India, US, etc.

The growing food demand and the treat of heavy crop losses due to global climate change impose the urgent development of strategies to substantially improve food availability. Enhancing crop productivity plays thus an important role in achieving breeder's goals. Agricultural production and yield are predicted to be affected by increasing temperatures resulting from global warming (Ainsworth \& Ort, 2010), and the most important goal of plant breeders is to obtain high yielding varieties that are resistant to biotic and abiotic stress factors. Plants possess various mechanisms to ensure survival under elevated temperatures. Molecular biologists can analyse these mechanisms and isolate their key features to facilitate implementation of tolerance traits into other plant species, for the benefit of plant breeders.

Cultivation of tomatoes under higher temperatures than the optimum has a negative impact on plant growth, decreasing productivity and ultimately yield (Sato et al., 2006). As a consequence of global warming, the impact of high temperatures on field-grown tomatoes has become a critical issue to resolve. A thorough understanding of mechanisms of heat tolerance and physiological responses in tomatoes is thus imperative for maintenance and development of future crop systems.

In addition to its worldwide cultivation and economic importance, tomato has several characteristics that makes it a convenient model plant species, such as a relatively compact genome $(950 \mathrm{Mb})$ combined with a marker-saturated genetic linkage map (http://solgenomics.net), rich germplasm collections (http://tgrc.ucdavis.edu ), highly efficient transformation protocols, diploid genetics and short reproduction period (Pujar et al., 2013). Moreover, the fully annotated genome sequence and the resequenced genomes of 84 tomato accessions and related wild species (The 100 Tomato Genome Sequencing et al., 2014) establishes tomato as a prominent model system for research into plant genetics and physiology. Tomato is likely to become an alternative model plant to Arabidopsis thaliana due to its diverse developmental traits not found in Arabidopsis, which include: the photoperiod-independent sympodial flowering and the formation of fleshy climacteric fruits, compound leaves, mycorrhizal roots and glandular trichomes (Carvalho et al., 2011). The plethora of mutants available in tomato is another advantageous characteristic of this model plant (Emmanuel and Levy, 2002), including Micro Tom, the model cultivar for tomato functional genomics (Sun et al., 2006). 


\section{Heat stress effects in tomato}

In virtually all crops species, including tomato, the reproductive development is more sensitive to high temperatures than the vegetative growth (Lobell and Field, 2007; Prasad et al., 2008), and not only severe heat stress but also a mild increase in temperature negatively affects fruit set. The male gametophyte is particularly sensitive to high temperatures at all stages of development, while the pistil and the female gametophyte are considered to be more tolerant (Zinn et al., 2010).

The developmental pathway for a pollen grain starts with the separation of the reproductive tissues of the anther, continues with meiosis of the pollen mother cell, followed by mitosis and microspore maturation that results in the mature pollen grain. After initiation, the highly specialized anther tissues will acquire non-reproductive (e.g., the tapetum for support) or reproductive functions (the pollen mother cell for pollen formation) as shown diagrammatically in Figure 1.

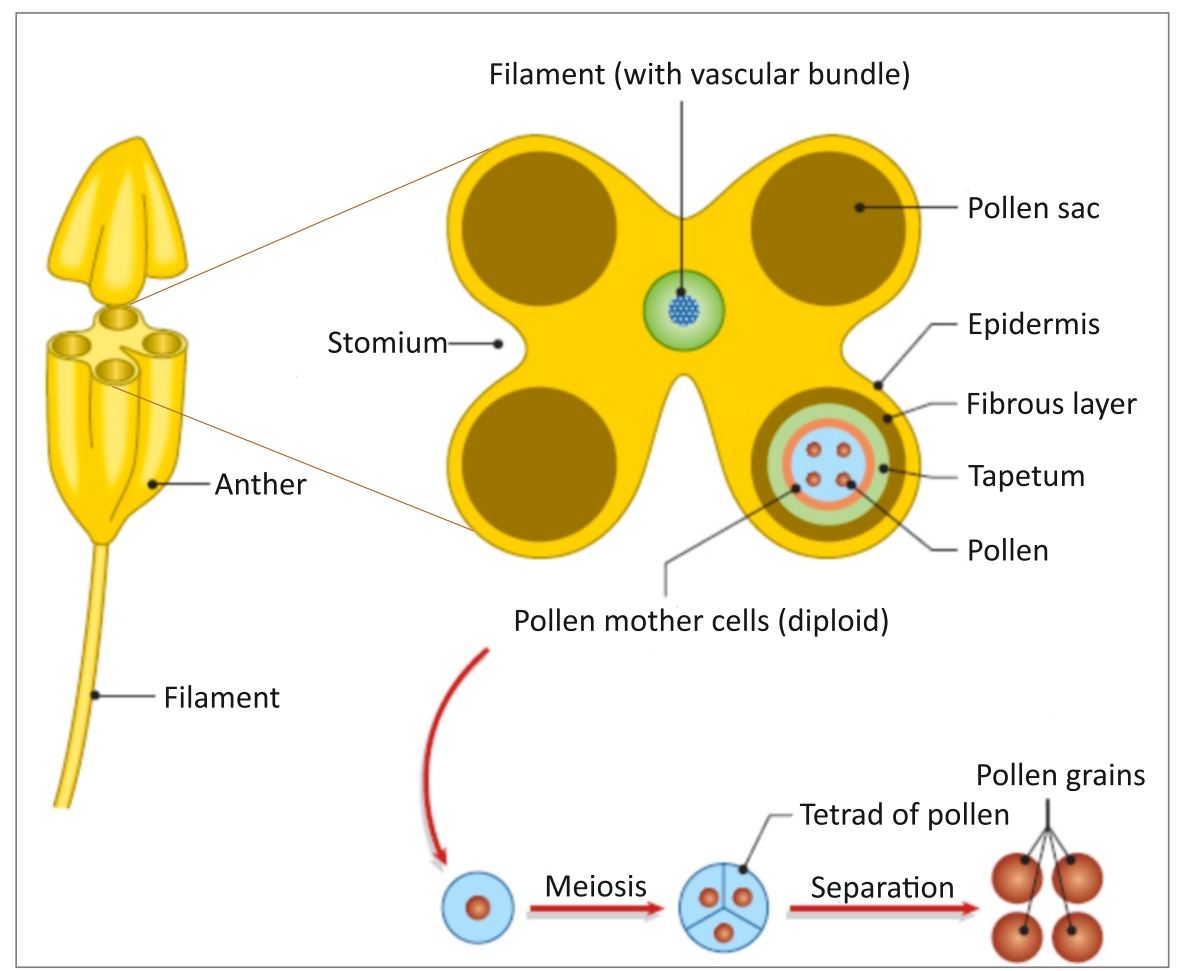

Figure 1. Schematic structure of anther and pollen grains development. A diagrammatic representation of a section through an anther is shown, together with the stages of pollen development from a microsporocyte to a mature microspore.

Tomato grows worldwide under diverse environmental conditions with optimal temperatures of $25-30^{\circ} \mathrm{C}$ day and $20^{\circ} \mathrm{C}$ night (Camejo et al., 2005) and also displays marked responses to heat (Abiko et al., 2005; Porch and Jahn, 2001). Fruit setting in tomato is reportedly interrupted at temperatures above $26 / 20^{\circ} \mathrm{C}$ day/night, respectively, and is often completely arrested above $38 / 27^{\circ} \mathrm{C}$ day/night (Levy et al., 1978). In many agricultural regions hot summers result in up to $70 \%$ harvest losses for cultivated tomatoes (Sato et al., 2002). Investigations to identify floral characteristics affected by high temperatures in tomato reveal many floral abnormalities such as stigma exertion without anthesis and persistent flowers without fruit-set, especially in heat sensitive genotypes. Sometimes anther cone splitting is also observed (Lohar and Peat, 1998). In addition to developmental abnormalities in male tissues, a reduced photosynthesis and hormonal balance in sink tissues have been related to tomato poor fruit set at high temperatures (Peet et al., 1998). Heat stress affects 
photosynthetic parameters such as chlorophyll a: b proportion, chlorophyll and carotenoids levels (Wahid, 2007) and disrupts the hydraulic conductivity of root and the leaf water relationships (Morales et al., 2003).

The plant growth regulator and stress hormone abscisic acid (ABA) has also been found to accumulate in plants on several types of abiotic stress, including heat, cold or drought (Zhang et al, 2006). ABA seems to regulate the osmotic stress signal transduction response and confer plant stress tolerance through the up-regulation of a large set of stress-responsive genes (Fujita et al, 2011) and interaction with the sugar signalling pathway (Dekkers B, 2008). Increases in ABA levels in the stamens have long been correlated with abnormalities in anther development leading to sterility (Singh and Sawhney, 1998). Endogenous ABA is now believed to act as a stress-responsive signal that induces pollen abortion by specifically repressing apoplastic sugar transport in the anther, as both cold- or drought-sensitive wheat and rice anthers accumulate high levels of ABA and show high levels of pollen abortion due to reduced sucrose transport and altered sucrose metabolism (De Storme and Geelen, 2014).

A comparison of the effects of heat stress on a heat-tolerant and a heat-sensitive tomato cultivar showed that temperature stress affected mainly the development of pollen grains, where reduced viability was more pronounced in the heat-sensitive cultivar (Firon et al., 2006). The major effect of heat stress on the pollen development process was the disruption of carbohydrate metabolism and proline translocation (Sato et al., 2006) resulting into a decrease in starch and sugar concentration in mature pollen grains with a subsequent loss of pollen viability (Pressman et al., 2002). More recently, the transcriptome profiling of heat stressed maturing tomato microspores revealed the involvement of heat shock transcription factors, ROS scavengers, sugars, plant hormones, transport and signalling related as well as many other regulatory genes (Frank et al., 2009).

\section{Heat stress tolerance in tomato}

A wide range of heat stress phenotypes has been described in tomato and physiological observations both under field and greenhouse conditions reveal a variable degree of tolerance between different commercial genotypes. (Levy et al., 1978; Sato et al., 2004).

The differences in pollen grain development in tolerant tomato genotypes are considered the most critical factors to determine fruit set under heat stress (Levy et al., 1978). A major difference between heat tolerant and heat sensitive tomato genotypes growing under heat stress conditions is the ability to accumulate starch and soluble sugars by the former during pollen development. As heattolerant cultivars appear to have a mechanism for maintaining the appropriate carbohydrate content under heat stress, the carbohydrate content of developing and mature pollen grains could be an important factor in determining pollen quality (Firon et al., 2006). Recent studies on a heat-tolerant tomato genotype demonstrated that high invertase activity and increased sucrose import into young tomato fruits contribute to heat tolerance through increasing sink strength and sugar signalling activities, possibly regulating a programmed cell death pathway (Li et al., 2012).

At molecular level, a comparative transcriptome analysis between tolerant and sensitive tomato genotypes associated the tolerance to heat with less and lower differentially expressed transcripts but with a higher heat shock gene induction in the anthers of heat-tolerant genotypes. Also interestingly, higher basal expression levels of heat shock, adaptation and carbohydrate metabolism related genes were measured for microspores of a heat-tolerant tomato cultivar compared with those of a heat-sensitive cultivar. (Bita et al., 2011; Frank et al., 2009). 


\section{Development of heat-stress tolerance in tomato}

Conventional breeding schemes have been extensively deployed for uncovering heat tolerance genes and their inheritance patterns to unravel their underlying molecular mechanism (Wahid et al. 2007, Farooq et al. 2011). DNA marker discovery and genotyping assays have permitted the accurate determination of chromosomal positions of the QTLs responsible for heat tolerance in different crops, including tomato (Grilli, 2007; Xiang-yang, 2008). Several efforts to promote tomato heat resistant lines through traditional plant breeding methods have become successful (Wahid et al., 2007), as conventional breeding offers little information on the chromosomal location or the gene(s) underlying the traits that control tolerance to stress (due to epistasis, pleiotropy or linkage), or on the genetic origin of yield related traits due to dominant or over-dominant effects (Semel et al., 2006).

The genetic variation in the ability of different tomato cultivars to set fruit under high temperature conditions could make selection for heat tolerance possible. In addition, it seems that the capacity for thermotolerance can be achieved by modulating the expression levels of such 'responsive' genes prior to heat stress exposure (Frank et al., 2009). Moreover, genes that confer stress tolerance could be sourced from germplasm collections, or wild relatives or organisms that live in extreme habitats (Abdul-Baki, 1991). For example, a tolerant wild tomato variety showed no changes in the net photosynthetic rate and no injury to the plasma membrane, but an increase in chlorophyll $\mathrm{a} / \mathrm{b}$ and a decrease in chlorophyll/carotenoid ratios when exposed to an acute heat stress of $2 \mathrm{~h}$ at $45^{\circ} \mathrm{C}$ (Camejo et al., 2005).

Transgenic technologies employing increasingly advanced transformation and regeneration protocols and gene editing techniques (CRISPR-CAS9) are also a key tool in designing tolerant crops via gene expression manipulation (Brooks et al., 2014). Experimental approaches to insert tolerance into tomato have been limited, compared to the experiments aimed at engineering drought, salt or cold stress tolerance (Marco et al., 2015). However, constitutive expression of various proteins has been shown to enhance heat tolerance in tomato. Engineering levels of metabolites, such as polyamines, ethylene, anti-oxidants and chloroplastic or heat shock proteins provide successful approaches to increase tolerance to heat stress in tomato (Cheng et al., 2009; Giorno et al., 2010; Li et al., 2010; Mahesh et al., 2013; Suzuki et al., 2005; Wang et al., 2006).

In order to meet the future requirements of agricultural production, a proper assessment of environmental stress tolerance for crops needs thus to be designed and implemented. The development of heat tolerant cultivars is crucial to ensure successful crop production during periods of climate change and variability. Breeding programs should identify such tolerance traits in available germplasm to contribute in selecting heat-tolerant cultivars (Figure 2). Multiple opportunities for improvement exist, as tolerance to high temperatures is a multigenic and complex network of chaperones and other protective proteins acting together to defend the cells from heat injury. 


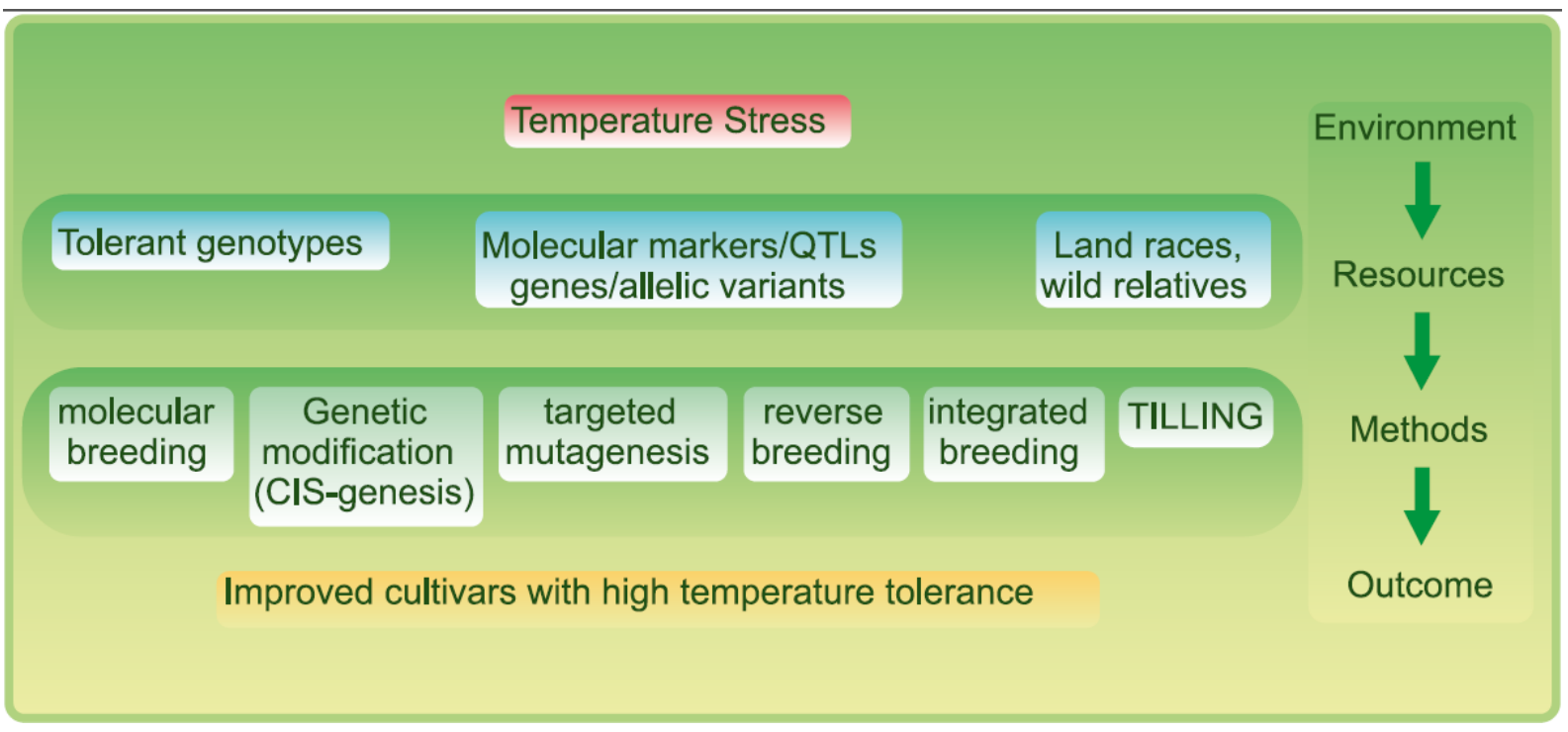

Figure 2. Methods of ameliorating heat stress in plants shown as a flowchart of resources, methods and possible outcomes.

\section{Aims and outline of the thesis}

Generally, tomato is considered sensitive to heat stress. During the development of the tomato male gametophyte, anther development and particularly the first stage of meiosis are the most sensitive phases. Our goal was to analyse the general molecular response to moderate heat stress (MHS) in tomato meiotic anthers and then characterise aspects of the response to heat in tolerant genotypes.

Understanding the metabolic changes that occur in heat tolerant varieties/lines during heat stress is essential for the production of tomato crops adapted to variable temperature increases.

First, cDNA-AFLP was employed to reveal a general spatial and temporal response to MHS in a standard cultivar. The results showed about $1 \%$ of the analysed genes having significant modulation (down-regulation) in expression after just $2 \mathrm{~h}$ of stress. Several transcripts were identified and associated to a response to heat. Additional analyses revealed that the candidate genes are expressed in the tapetum, but also in other tissues (Chapter 3).

The Combimatrix technology has been used to fingerprint differences in response to heat between a tolerant and a sensitive genotype, with the aim of detecting candidate tolerance genes. The microarray analysis also shows about $1 \%$ of the genes having a significant modulation in expression after $2 \mathrm{~h}$ of stress, and that the tolerant genotype reacts with fewer transcriptomic changes and exhibiting high constitutive levels of transcripts involved in protection and thermotolerance. In the heat-tolerant genotype, the majority of changes in gene expression is represented by up-regulation, while in the heat-sensitive genotype there is a general trend to down-regulate gene expression, soon after MHS. The putative functions associated with the genes identified by cDNA-AFLP or microarrays indicate involvement of heat shock, metabolism, antioxidant and development pathways. Based upon the observed differences in response to MHS and on literature sources, we identified a number of candidate transcripts involved in heat-tolerance. A functional analysis of expression modulation by qRT-PCR was performed in several pairs of genotypes and the results confirm observations from microarray regarding gene modulation (Chapter 4). An experimental approach to obtain insight into the hormonal regulation of the response to heat is described in chapter 5 . Additional observations and results are discussed in chapter 6. 


\section{References}

Abdul-Baki, A.A. (1991) Tolerance of Tomato Cultivars and Selected Germplasm to Heat Stress. Journal of the American Society for Horticultural Science 116, 1113-1116.

Abiko, M., Akibayashi, K., Sakata, T., Kimura, M., Kihara, M., Itoh, K., Asamizu, E., Sato, S., Takahashi, H. and Higashitani, A. (2005) High-temperature induction of male sterility during barley (Hordeum vulgare L.) anther development is mediated by transcriptional inhibition. Sexual Plant Reproduction 18, 91-100.

Bita, C.E., Zenoni, S., Vriezen, W.H., Mariani, C., Pezzotti, M. and Gerats, T. (2011) Temperature stress differentially modulates transcription in meiotic anthers of heat-tolerant and heatsensitive tomato plants. BMC genomics 12, 384.

Brooks, C., Nekrasov, V., Lippman, Z.B. and Van Eck, J. (2014) Efficient gene editing in tomato in the first generation using the clustered regularly interspaced short palindromic repeats/CRISPR-associated9 system. Plant physiology 166, 1292-1297.

Camejo, D., Rodriguez, P., Morales, M.A., Dell'Amico, J.M., Torrecillas, A. and Alarcon, J.J. (2005) High temperature effects on photosynthetic activity of two tomato cultivars with different heat susceptibility. Journal of plant physiology 162, 281-289.

Carvalho, R.F., Campos, M.L., Pino, L.E., Crestana, S.L., Zsogon, A., Lima, J.E., Benedito, V.A. and Peres, L.E. (2011) Convergence of developmental mutants into a single tomato model system: 'Micro-Tom' as an effective toolkit for plant development research. Plant methods 7, 18.

Cheng, L., Zou, Y., Ding, S., Zhang, J., Yu, X., Cao, J. and Lu, G. (2009) Polyamine accumulation in transgenic tomato enhances the tolerance to high temperature stress. Journal of integrative plant biology 51, 489-499.

De Storme, N. and Geelen, D. (2014) The impact of environmental stress on male reproductive development in plants: biological processes and molecular mechanisms. Plant, cell \& environment 37, 1-18.

Dekkers B, S.J., Smeekens S (2008) Interaction between sugar and abscisic acid signalling during early seedling development in Arabidopsis. . Plant molecular biology 67.

Emmanuel, E. and Levy, A.A. (2002) Tomato mutants as tools for functional genomics. Current opinion in plant biology 5, 112-117.

Firon, N., Shaked, R., Peet, M., Pharr, D., Zamski, E., Rosenfeld, K., Althan, L. and Pressman, E. (2006) Pollen grains of heat tolerant tomato cultivars retain higher carbohydrate concentration under heat stress conditions. Scientia Horticulturae 109, 212-217.

Frank, G., Pressman, E., Ophir, R., Althan, L., Shaked, R., Freedman, M., Shen, S. and Firon, N. (2009) Transcriptional profiling of maturing tomato (Solanum lycopersicum L.) microspores reveals the involvement of heat shock proteins, ROS scavengers, hormones, and sugars in the heat stress response. Journal of experimental botany 60, 3891-3908.

Giorno, F., Wolters-Arts, M., Grillo, S., Scharf, K.D., Vriezen, W.H. and Mariani, C. (2010) Developmental and heat stress-regulated expression of HsfA2 and small heat shock proteins in tomato anthers. Journal of experimental botany 61, 453-462.

Grilli, G.V.G., L. T. Braz, and E. G. M. Lemos (2007) QTL identification for tolerance to fruit set in tomato by FAFLP markers. Crop Breeding and Applied Biotechnology 7.

Levy, A., Rabinowitch, H. and Kedar, N. (1978) Morphological and physiological characters affecting flower drop and fruit set of tomatoes at high temperatures. Euphytica 27, 211-218.

Li, F., Wu, Q.-Y., Sun, Y.-L., Wang, L.-Y., Yang, X.-H. and Meng, Q.-W. (2010) Overexpression of chloroplastic monodehydroascorbate reductase enhanced tolerance to temperature and methyl viologen-mediated oxidative stresses. Physiologia plantarum 139, 421-434.

Li, Z., Palmer, W.M., Martin, A.P., Wang, R., Rainsford, F., Jin, Y., Patrick, J.W., Yang, Y. and Ruan, Y.L. (2012) High invertase activity in tomato reproductive organs correlates with enhanced sucrose import into, and heat tolerance of, young fruit. Journal of experimental botany 63, 1155-1166.

Lobell, D.B. and Field, C.B. (2007) Global scale climate-crop yield relationships and the impacts of recent warming. Environmental Research Letters 2, 014002. 
Lohar, D. and Peat, W. (1998) Floral characteristics of heat-tolerant and heat-sensitive tomato $(<\mathrm{i}>$ Lycopersicon esculentum $<$ i $>$ Mill.) cultivars at high temperature. Scientia Horticulturae 73, 53-60.

Mahesh, U., Mamidala, P., Rapolu, S., Aragao, F.J.L., Souza, M.T., Rao, P.J.M., Kirti, P.B. and Nanna, R.S. (2013) Constitutive overexpression of small HSP24. 4 gene in transgenic tomato conferring tolerance to high-temperature stress. Molecular Breeding, 1-11.

Marco, F., Bitrián, M., Carrasco, P., Rajam, M.V., Alcázar, R. and Tiburcio, A.F. (2015) Genetic engineering strategies for abiotic stress tolerance in plants. In: Plant Biology and Biotechnology pp. 579-609. Springer.

Morales, D., Rodríguez, P., Dell'Amico, J., Nicolás, E., Torrecillas, A. and Sánchez-Blanco, M.J. (2003) High-Temperature Preconditioning and Thermal Shock Imposition Affects Water Relations, Gas Exchange and Root Hydraulic Conductivity in Tomato. Biologia Plantarum 47, 203-208.

Peet, M.M., Sato, S. and Gardner, R.G. (1998) Comparing heat stress effects on male-fertile and male-sterile tomatoes. Plant Cell and Environment 21, 225-231.

Porch, T. and Jahn, M. (2001) Effects of high-temperature stress on microsporogenesis in heatsensitive and heat-tolerant genotypes of Phaseolus vulgaris. Plant, cell \& environment 24, 723-731.

Prasad, P., Pisipati, S., Mutava, R. and Tuinstra, M. (2008) Sensitivity of grain sorghum to high temperature stress during reproductive development. Crop Sci 48, 1911-1917.

Pressman, E., Peet, M.M. and Pharr, D.M. (2002) The effect of heat stress on tomato pollen characteristics is associated with changes in carbohydrate concentration in the developing anthers. Annals of botany 90, 631-636.

Pujar, A., Menda, N., Bombarely, A., Edwards, J.D., Strickler, S.R. and Mueller, L.A. (2013) From manual curation to visualization of gene families and networks across Solanaceae plant species. Database : the journal of biological databases and curation 2013, bat028.

Sato, S., Kamiyama, M., Iwata, T., Makita, N., Furukawa, H. and Ikeda, H. (2006) Moderate increase of mean daily temperature adversely affects fruit set of Lycopersicon esculentum by disrupting specific physiological processes in male reproductive development. Annals of botany 97, 731-738.

Sato, S., Peet, M.M. and Gardner, R.G. (2004) Altered flower retention and developmental patterns in nine tomato cultivars under elevated temperature. Scientia Horticulturae 101, 95-101.

Sato, S., Peet, M.M. and Thomas, J.F. (2002) Determining critical pre-and post-anthesis periods and physiological processes in Lycopersicon esculentum Mill. exposed to moderately elevated temperatures. Journal of experimental botany 53, 1187-1195.

Semel, Y., Nissenbaum, J., Menda, N., Zinder, M., Krieger, U., Issman, N., Pleban, T., Lippman, Z., Gur, A. and Zamir, D. (2006) Overdominant quantitative trait loci for yield and fitness in tomato. Proceedings of the National Academy of Sciences of the United States of America 103, 12981-12986.

Singh, S. and Sawhney, V. (1998) Abscisic acid in a male sterile tomato mutant and its regulation by low temperature. Journal of experimental botany 49, 199-203.

Sun, H.J., Uchii, S., Watanabe, S. and Ezura, H. (2006) A highly efficient transformation protocol for Micro-Tom, a model cultivar for tomato functional genomics. Plant \& cell physiology 47, 426-431.

Suzuki, N., Rizhsky, L., Liang, H., Shuman, J., Shulaev, V. and Mittler, R. (2005) Enhanced tolerance to environmental stress in transgenic plants expressing the transcriptional coactivator multiprotein bridging factor 1c. Plant physiology 139, 1313-1322.

The 100 Tomato Genome Sequencing, C., Aflitos, S., Schijlen, E., de Jong, H., de Ridder, D., Smit, S., Finkers, R., Wang, J., Zhang, G., Li, N., Mao, L., Bakker, F., Dirks, R., Breit, T., Gravendeel, B., Huits, H., Struss, D., Swanson-Wagner, R., van Leeuwen, H., van Ham, R.C.H.J., Fito, L., Guignier, L., Sevilla, M., Ellul, P., Ganko, E., Kapur, A., Reclus, E., de Geus, B., van de Geest, H., te Lintel Hekkert, B., van Haarst, J., Smits, L., Koops, A., Sanchez-Perez, G., van Heusden, A.W., Visser, R., Quan, Z., Min, J., Liao, L., Wang, X., Wang, G., Yue, Z., Yang, X., Xu, N., Schranz, E., Smets, E., Vos, R., Rauwerda, J., Ursem, 
R., Schuit, C., Kerns, M., van den Berg, J., Vriezen, W., Janssen, A., Datema, E., Jahrman, T., Moquet, F., Bonnet, J. and Peters, S. (2014) Exploring genetic variation in the tomato (Solanum section Lycopersicon) clade by whole-genome sequencing. The Plant Journal 80, 136-148.

Wahid, A. (2007) Physiological implications of metabolite biosynthesis for net assimilation and heatstress tolerance of sugarcane (Saccharum officinarum) sprouts. Journal of plant research 120, 219-228.

Wahid, A., Gelani, S., Ashraf, M. and Foolad, M. (2007) Heat tolerance in plants: an overview. Environmental and Experimental Botany 61, 199-223.

Wang, Y., Wisniewski, M., Meilan, R., Cui, M. and Fuchigami, L. (2006) Transgenic tomato (Lycopersicon esculentum) overexpressing cAPX exhibits enhanced tolerance to UV-B and heat stress. J Appl Hort 8, 87-90.

Xiang-yang, X., W. Dong-mei, K. Li-gong, L. Jing-fu (2008) Selection of SSR and RAPD Markers Related to Tomato Heat Tolerance. Acta Hort. Sinica. 35.

Zinn, K.E., Tunc-Ozdemir, M. and Harper, J.F. (2010) Temperature stress and plant sexual reproduction: uncovering the weakest links. Journal of experimental botany 61, 1959-1968. 


\section{Chapter 2}

\section{Plant tolerance to high temperature and production of heat stress tolerant crops}

Published as:

Craita Bita and Tom Gerats (2013) "Plant tolerance to high temperature in a changing environment: scientific fundamentals and production of heat stress tolerant crops “ in Front. Plant Sci. | doi: 10.3389/fpls.2013.00273 


\section{Climate change undercuts global food security}

Abiotic stresses are often interrelated, but either singularly or in combination, they cause morphological, physiological, biochemical and molecular changes that adversely affect plant growth and productivity, and ultimately yield. Heat, drought, cold and salinity are the major abiotic stresses that induce severe cellular damage in plant species, including crop plants. Fluctuations in temperature occur naturally during plant growth and reproduction. However, extreme variations during hot summers can damage the fine intermolecular interactions needed for proper growth, impairing thus plant development and fruit-set. The increasing threat of climate change is already having a substantial impact on agricultural production worldwide as heat waves cause significantly yield losses with great risks for future global food security (Christensen and Christensen, 2007).

Climatological extremes including very high temperatures are predicted to have a general negative effect on plant growth and development, leading to catastrophic loss of crop productivity and resulting in wide spread famine. Future agricultural production and thus global food security will encounter additional challenges from human population growth (Figure 1).

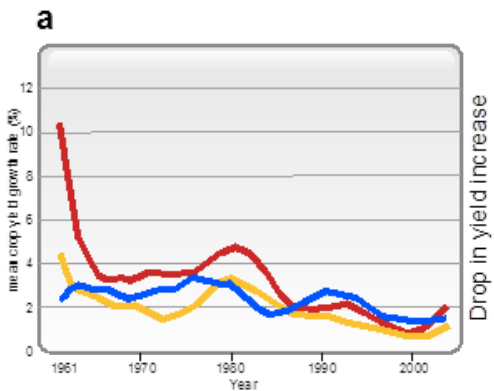

d

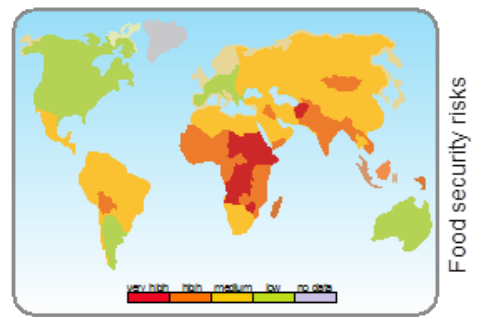

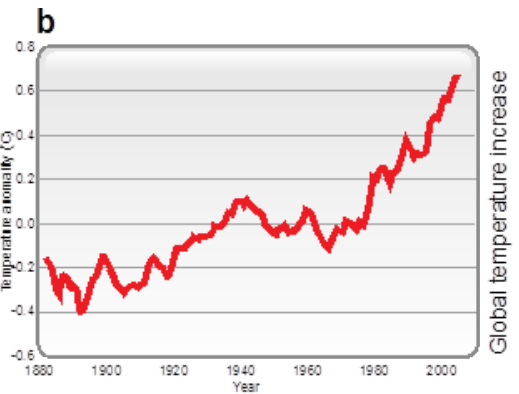

e

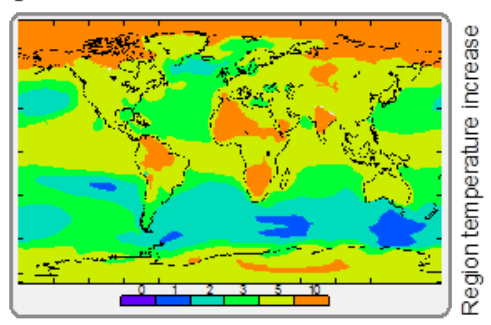

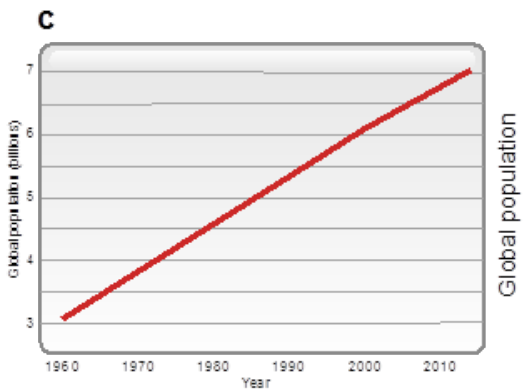

f

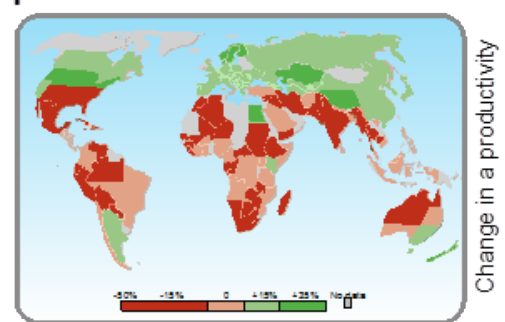

Figure 1. Global temperature and population trends. (a) Reduction in yield increases of major crops since 1961; wheat (red graph), rice (yellow graph), maize (blue graph). Data taken from FAO-STAT (http://faostat.fao.org). (b) Global increases in temperature since 1880. Data taken from (Hansen et al, 2012) (c) Population increase since 1960. Data taken from the US Census Bureau (http://www.census.gov) (d) Regional food security risk areas. Data taken from http://maplecroft.com (e) Regional temperature increase. Data taken from the Hadely Centre, UK Met Office (http://www.metoffice.gov.uk) (f) Changes in regional agricultural productivity. Data taken from the Bard Center for Environmental Policy (http://www.bard.edu).

Presently, the Indian lowlands are the source of approximately $15 \%$ of global wheat production but it is anticipated that climate changes will transform these into a heat-stressed, shortseason production environment. In a similar manner, a temperature increase of 3-4 $\mathrm{C}$ could cause crop yields to fall by $15-35 \%$ in Africa and Asia and by $25-35 \%$ in the Middle East (Ortiz et al., 2008). Based on a mathematical modelling, cereal production in Southeast Asia and Southern Africa is most likely to be affected by the climate change in case new strategies for amelioration are not found (Fischer and Edmeades, 2010; Nelson, 2009). Temperatures during the growing season in the tropics and subtropics may even exceed the most extreme seasonal temperatures observed till now, which will further aggravate land degradation (Battisti and Naylor, 2009; Varshney et al., 2011). Latin 
America is predicted to experience increases in temperatures and decreases in precipitations throughout the continent but especially in Central America and the Caribbean. In Europe, most of the temperature increases will be in Southern and Central parts, the most affected countries being Spain, Portugal and Italy. However, the changing climate conditions will potentially expand agricultural areas in the northern countries, where today crop cultivation is limited due to low temperature. In North America for example, the largest increase in temperature is expected at latitudes over $50^{\circ} \mathrm{N}$, e.g. in Canada (Lotze-Campen, 2011).

Populations from developing countries are likely to be the most seriously affected as nearly $50 \%$ rely entirely on agriculture. In addition, $75 \%$ of the world's poor live in rural areas. Thus, as population expands, crop production will have to be tailored to sustain food security and it has been suggested that world food production will have to increase by $70 \%$ to meet the demand of an expected population of 9 billion in 2050 .

Despite the predicted increase in global food production, at present there is a major global food deficit and the relative rates of yield increase for the major cereal crops are declining (Easterling and Apps, 2005; Fischer and Edmeades, 2010) (Figure 1). In many crop species, the effects of high temperature stress are more prominent on reproductive development than on vegetative growth and the sudden decline in yield with temperature is mainly associated with pollen infertility (Young et al., 2004; Zinn et al., 2010). Adding in the surging demand for food from the emerging economies such as China and India reveals an even more extreme challenge for plant breeders and farmers to such an extent that by 2050 , the expected decline in calorie availability will aggravate malnutrition in children by 20\% (Chhetri and Chaudhary, 2011; Nelson, 2009). The growing food demand and the treat of heavy crop losses due to global climate change impose the urgent development of strategies to substantially improve food availability. Enhancing crop productivity plays thus an important role in achieving breeder's goals.

\section{Molecular, cellular and physiological impacts of heat stress in plants}

Of the major forms of abiotic stress plants are exposed to in nature, heat stress has an independent mode of action on the physiology and metabolism of plant cells. Although frequently, heat stress is compounded by additional abiotic stresses such as drought and salt stress, it is important to unravel the independent action and biological consequences of high temperature in order to ameliorate the effects of combined abiotic stress. The susceptibility to high temperatures in plants varies with the stage of plant development, heat stress affecting to a certain extent all vegetative and reproductive stages. The observed effects depend on species and genotype, with abundant inter- and intra-specific variations (Barnabás et al., 2008; Sakata and Higashitani, 2008). Various physiological injuries have been observed under elevated temperatures, such as scorching of leaves and stems, leaf abscission and senescence, shoot and root growth inhibition or fruit damage, which consequently lead to a decreased plant productivity (Vollenweider and Günthardt-Goerg, 2005).

Heat stress induces changes in respiration and photosynthesis and thus leads to a shortened life cycle and diminished plant productivity (Barnabás et al., 2008). The early effects of thermal stress comprise of structural alterations in chloroplast protein complexes and reduced activity of enzymes (Ahmad et al., 2010). In addition, by causing injuries to the cell membrane, organization of microtubules and ultimately to the cytoskeleton, heat stress changes membrane permeability and alters cell differentiation, elongation and expansion (Potters et al., 2008; Rasheed, 2009; Smertenko et al., 1997). For example, the photochemical modifications in the carbon flux of the chloroplast stroma and those of the thylakoid membrane system are considered the primary sites of heat injury (Wise et al., 2004), as photosynthesis and the enzymes of the Calvin-Benson cycle, including ribulose 1,5- 
bisphosphate carboxylase (Rubisco) and Rubisco activase are very sensitive to increased temperature and are severely inhibited even at low levels of heat stress, (Maestri et al., 2002; Morales et al., 2003).

A specific effect of high temperatures on photosynthetic membranes includes the swelling of grana stacks and an aberrant stacking. Such structural changes are accompanied by ion-leakage from leaf cells exposed to heat and changes in energy allocation to the photosystems (Allakhverdiev et al., 2008; Wahid and Shabbir, 2005). The maintenance of cellular membrane function under high temperature stress is thus essential for a sustained photosynthetic and respiratory performance (Chen et al., 2010). The detrimental effects of heat on chlorophyll and the photosynthetic apparatus are also associated with the production of injurious reactive oxygen species (ROSs) (Camejo et al., 2006; Guo et al., 2007). By increasing chlorophyllase activity and decreasing the amount of photosynthetic pigments, heat stress ultimately reduces the plant photosynthetic and respiratory activity (Sharkey and Zhang, 2010; Todorov et al., 2003). With respect to reproductive success, a decline of photosynthesis will eventually result in limited resource availability for reproduction in parental and gametophytic tissues due to a reduction in energy reserves leading to plant starvation (Sumesh et al., 2008; Young et al., 2004).

Homeostasis in general, including biosynthesis and compartmentalization of metabolites, is disturbed in high temperature challenged plant tissues (Maestri et al., 2002). High temperature modifies the activities of carbon metabolism enzymes, starch accumulation and sucrose synthesis, by down-regulating specific genes in carbohydrate metabolism (Ruan et al., 2010). Among the primary metabolites accumulating in response to heat stress are proline, glycine-betaine or soluble sugars (Wahid, 2007). Many plant species accumulate other osmolytes as well, such as sugar alcohols (polyols), or tertiary and quaternary ammonium compounds (Sairam and Tyagi, 2004). Osmolyte production under heat stress is thought to increase protein stability and stabilize the structure of the membrane bilayer (Mirzaei et al., 2012; Sung et al., 2003). Secondary metabolites such as phenolics including flavonoids, anthocyanin and plant steroids, are also significantly involved in plant tissues responses under heat stress and play a variety of roles in abiotic stress responses, generally associated with tolerance (Wahid, 2007).

Several key phyto-hormones including abscisic acid (ABA), salicylic acid (SA), and ethylene (ET) also increase their levels under heat stress, while others decrease, such as cytokinin (CK), auxin (AUX) and gibberellic acids (GAs), fluctuations that ultimately cause premature plant senescence (Larkindale et al., 2005; Larkindale and Huang, 2004; Talanova et al., 2003). For example, the abscission of reproductive organs, an important effect of heat stress, is known to be caused by increased ABA and ET levels and reduced levels and transport of AUXs (Binder and Patterson, 2009). Similarly, an altered AUX biosynthesis in developing anthers was suggested to be related to pollen sterility (Sakata et al., 2010). Additionally, a comparable variation in CK content was also found to be the cause of reduced kernel filling in cereals (Banowetz et al., 1999).

Approximately $5 \%$ of the plant transcriptome is up-regulated two-fold or more in response to heat stress in plants, and although often greatly induced by heat stress, chaperones are only a minor part of the general heat shock response (Larkindale and Vierling, 2008; Saidi et al., 2011). Most of the transcripts represent genes acting in primary and secondary metabolism, translation, transcription, regulation and responses to environmental stresses, and in processes such as calcium and phytohormone signalling, sugar and lipid signalling or protein phosphorylation.

The changes in ambient temperature are sensed by plants with a complicated set of sensors positioned in various cellular compartments. Membrane fluidity changes as a result of high temperatures seem to play an important role in temperature sensing. The increased fluidity of the membrane leads to leads to activation of lipid-based signaling cascades and to an increased $\mathrm{Ca} 2+$ influx and cytoskeletal reorganization. The influx is relative to the heat stress intensity and determines the extent of the subsequent heat shock protein (HSP) synthesis while calmodulin increase the 
transcriptional activity of the heat shock factors (HSFs), resulting in the up regulation of MAPKs and calcium dependent protein kinase (CDPK) (Frank et al., 2009). Signaling between these routes leads to the production of osmolytes and antioxidants for cell water balance and osmotic adjustment in response to heat stress. Recently it was shown that differences in the tissue specific activation of various signalling pathways may occur between vegetative and reproductive tissues (Mittler et al., 2011).

Analogous to other abiotic stresses, heat stress results in the production of ROSs and invokes oxidative stress responses (Potters et al., 2007). Generating activated oxygen species under heat stress is a symptom of cellular damage, where membrane lipids and pigments peroxidation compromise membrane permeability and function. ROS cause damage to a wide range of cellular components such as the photosynthetic apparatus and various other components, hindering thus metabolic activities and affecting plant growth and yield by limiting metabolic flux activities (Sairam and Tyagi, 2004; Xu et al., 2006). The ROS related impairment of important mitochondrial and chloroplast electron transport chains associated with carbon metabolism results in reduced power and energy production (Foyer and Noctor, 2009). Although ROSs are clearly a direct cause of cellular damage on multiple levels, several studies have also shown that ROS play a key role as molecular signals, linking plant responses to pathogen infection, environmental stresses, PCD and even developmental stimuli (Gechev et al., 2006). ROS/redox signalling networks in the chloroplast and mitochondria have important roles in plant adaption to abiotic stresses. These signals contribute to a complex interplay between organelles homeostasis under stress conditions and different cellular components and by controlling essential processes such as transcription, translation, energy metabolism and protein phosphorylation (Mittler et al., 2011).

Subsequently, ROS production contributes to the transduction of the heat signal and expression of heat shock genes (Königshofer et al., 2008). Heat stress results in the misfolding of newly synthesized proteins and the denaturation of existing proteins. Protein thermostability is believed to be provided in part by chaperones, a specific class of proteins capable of assisting other proteins in proper post-translational folding and in maintaining them in a functional state (Ellis, 1990). In standard growth conditions the HSPs control cellular signalling, protein folding, translocation and degradation, but under heat stress they prevent protein misfolding and aggregation and also act to protect cellular membranes. An increased production of HSPs occurs when plants experience either abrupt or gradual increases in temperature resulting in heat stress (Nover et al., 2001). There are considerable variations in the pattern of HSP gene expression in different species and even among genotypes within species. Heat shock factors (HSF) are the transcriptional activators of HSPs (Banti et al., 2010). HSF regulation in Arabidopsis was shown to be positively regulated by small HSP and co-chaperones of the HP90 complex such as ROF1, and negatively affected by an HSF binding protein (Meiri et al., 2010; Scharf et al., 2011).

A class of bHLH transcription factors known as phytochrome interacting factors (PIFs) has also been connected in the heat related signalling mechanisms. PIFs have a wide range of regulatory roles in photomorphogenesis, skotomorphogenesis and the down-stream regulation of hormone levels, in particular AUX and GA and implicitly on other phytohormones such as ABA. The precise role of these proteins in heat stress remains unclear; however the transcriptional response of PIF4 in particular has clear knock-on effects on early stages of plant development (Leivar and Quail, 2011).

Heat stress also leads to the transient activation of repetitive elements or silenced gene clusters close to the centromeric regions as well as the transient loss of epigenetic gene silencing (Lang-Mladek et al., 2010; Pecinka et al., 2010). Such gene silencing mechanisms are thought to be involved in transcriptional repression by hetero-chromatinization of repetitive DNA regions in plants (Khraiwesh et al., 2012). Recent studies have indicated that regulation of stress responsive genes often depends on chromatin remodelling (Figure 2). 


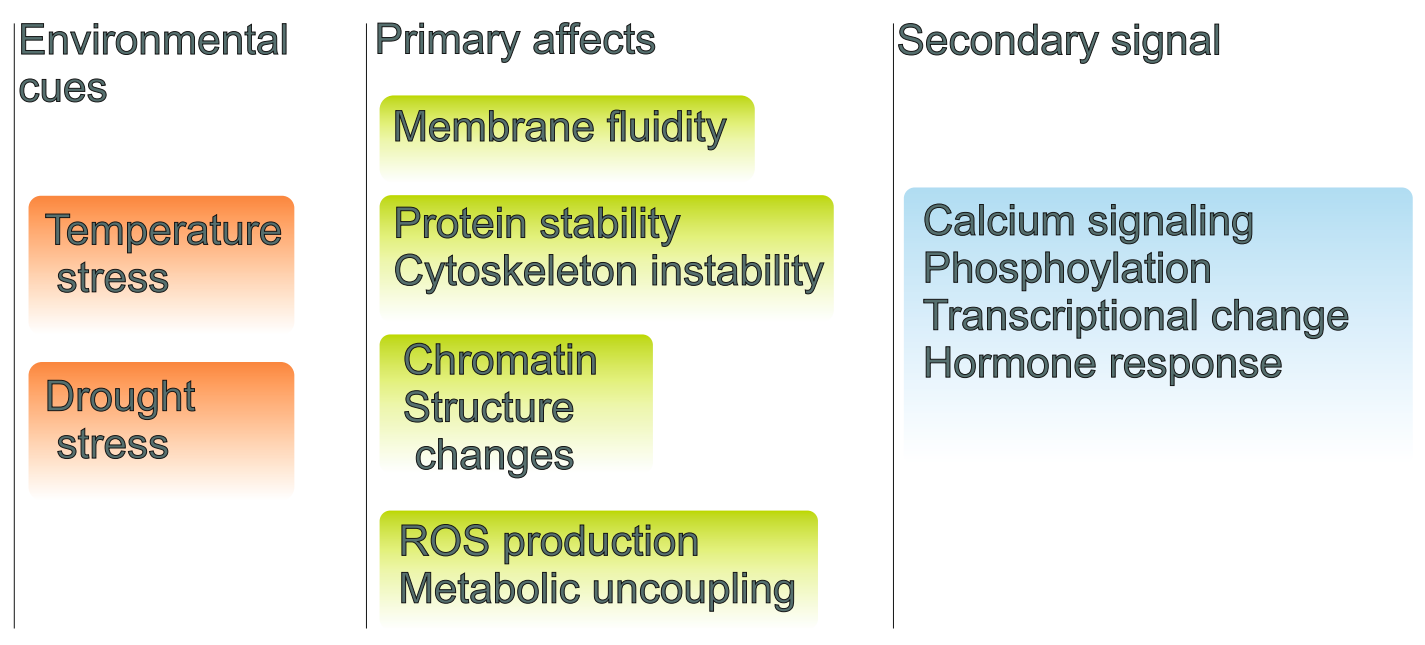

Figure 2. Environmental signalling pathways with external cues and internal repercussions. Environmental cues are on the left in red backgrounds with initial cellular effects with green backgrounds and the organismal signals are shown with blue background.

High temperatures cause transcriptional repression of genes involved in cell growth, such as histones and DNA polymerases and deregulation of DNA methylation and transposon activation (Pecinka et al., 2010; Sakata and Higashitani, 2008; Smith and Workman, 2012). For example, AtCHR12, a SNF2/Brahma (BRM)- type chromatin remodelling factor in Arabidopsis was shown to play a role in mediating the temporary growth arrest in response to drought and heat stress (Mlynárová et al., 2007). In addition, whereas histone modifications show only minor variations upon heat stress, there is evidence for a dramatic reduction in the number of nucleosomes associated with DNA, leading to loss of chromocenter organization, and this reduction in nucleosome density occurs throughout the genome. Efficient re-silencing of some of these activated targets seems to require the Chromatin Assembly Factor 1 (CAF-1) complex (Larkindale et al., 2005). Further support for the important role of histone-mediated transcriptional regulation in the temperature response comes from the recent discovery of the histone H2A.Z variant, involved in the regulation of the temperature transcriptome in Arabidopsis. H2A.Z nucleosomes wrap DNA more tightly, which influences the ability of RNA polymerase ( $\mathrm{Pol}$ ) II to transcribe genes in response to temperature, suggesting a mechanism by which the transcriptome can be thermally regulated (Kumar and Wigge, 2010).

A wide range of plant developmental and physiological processes is negatively affected by heat stress. When the stress occurs at key developmental stages such as reproduction, this becomes one of the major constraints of plant adaptation to a changing environment (Hall, 2001). For example, high temperature during wheat reproductive development hastened the decline in photosynthesis and leaf area, decreased shoot and grain mass as well as weight and sugar content of kernels, while also reducing water-use efficiency (Shah and Paulsen, 2003).

Sexual reproduction and flowering in particular have been long recognized as extremely sensitive to heat stress, which often results in reduced crop plant productivity (Hedhly et al., 2009; Thakur et al., 2010). Studies carried out under glass and climate chambers suggest that high temperature is most deleterious at the stage of flower bud initiation, and that this sensitivity is maintained for 10-15 days (Hedhly et al., 2009; Nava et al., 2009). Many legumes and cereals show a high sensitivity to heat stress during flowering and severe reductions in fruit set have also been shown for several temperate and even tropical fruit trees species (Frank et al., 2009; Saha et al., 2010), most 
probably as result of reduced water and nutrient transport during reproductive development (Young et al., 2004).

The male gametophyte is particularly sensitive to high temperatures at all stages of development, while the pistil and the female gametophyte are considered to be more tolerant (Hedhly, 2011). Heat stress often accelerates rather than delays the onset of anthesis, which means that the reproductive phase of development will be initiated prior to the accumulation of sufficient resources (Zinn et al., 2010). The reproductive development in angiosperms takes place within two floral organs, the male stamen and the female pistil (Ahmad et al., 2010; Pachauri, 2007). The developmental pathway for the male gametophyte (the pollen grain) starts with the separation of the reproductive tissues of the anther, continues with meiosis of the pollen mother cell, followed by mitosis and microspore maturation that results in the mature pollen grain. After initiation, the highly specialized anther tissues will acquire non-reproductive (e.g., the tapetum for support, the stomium for dehiscence) or reproductive functions (the pollen mother cell for pollen formation). Both tapetum and microspore development are essential for male fertility, as documented by numerous studies on male sterile mutants (Ainsworth et al., 2008; Christensen and Christensen, 2007; Jaggard et al., 2010; Prasad et al., 2003; Tauber et al., 2007; Young et al., 2004; Zinn et al., 2010). Major variations in gene expression are conveyed under high temperature stress resulting in tapetum degeneration and pollen sterility in several plant species (Endo et al., 2009; Oshino et al., 2007)

Male sterility as a consequence of heat stress can be widely observed among many sensitive crop plants and the impairment of pollen development has been the main factor involved in reduced yield under heat stress (Sakata and Higashitani, 2008; Wassmann et al., 2009). For example, in barley and Arabidopsis anthers developing under high temperature $\left(30^{\circ}-35^{\circ} \mathrm{C}\right)$, cell-proliferation is arrested, vacuoles are distended, chloroplast development is altered and mitochondrial abnormalities occur (Sakata et al., 2010). With regards to pollen viability, heat stress reduces carbohydrate accumulation in pollen grains and in the stigmatic tissue by altering assimilate partitioning and changing the balance between symplastic and apoplastic loading of the phloem (Taiz and Zeiger, 2006). Heat stress down-regulates sucrose synthase and several cell wall and vacuolar invertases in the developing pollen grains; as consequence, sucrose and starch turnover are disrupted and thus soluble carbohydrates accumulate in reduced levels (Sato et al., 2006). For example, in cowpea plants experiencing heat stress, drops in the concentration of soluble sugars in the anther walls, developing pollen grains and in the locular fluid result in decreased sugar concentration in the mature pollen grains and decreased pollen viability (Ismail and Hall, 1999). In tomato, the reduction of sink- and source-strength even under moderately elevated temperatures leads to a depletion in available carbohydrates at critical stages of plant development, leading to reduced fruit-set and other yield related parameters (Sato et al., 2006). In sorghum, heat stress reduces the accumulation of carbohydrate in pollen grains and ATP in the stigmatic tissue (Jain et al., 2007).

Heat stress also induces early abortion of tapetal cells which cause the pollen mother cells to rapidly progress towards meiotic prophase and finally undergo programed cell death (PCD), leading thus to pollen sterility (Oshino et al., 2007; Parish et al., 2012; Sakata and Higashitani, 2008). For example, under high temperature conditions, the structural abnormalities observed in developing microspores of snap bean anthers have been associated with tapetal degeneration due to malformations in the endoplasmic reticulum (Suzuki et al., 2001). Reduced dehiscence of tomato anthers under heat stress is also accompanied by closure of the locules and thus reduced pollen dispersal in several crop plants. (Peet et al., 2002). We have also shown a number of such phenotypes in tomato flowers (Figure 3). We have shown differential effects of high temperature on the development of tomato flowers, anthers and the viability of tomato pollen in heat sensitive and heattolerant lines. 
Continuing heat stress beyond a successful fertilization can also halt further development of the embryo (Barnabás et al., 2008). Heat stress during seed development may result in reduced germination and loss of vigour, leading hence to reduced emergence and seedling establishment, as it has been shown for several crop plants (Akman, 2009; Ren et al., 2009). In many temperate cereal crops both grain weight and grain number appear to be impacted by heat stress, with a decline in grain number directly proportional with increasing temperatures during flowering and grain filling (Mahmood et al., 2010; Porter and Semenov, 2005). Quality reductions in starch, protein and total oil yield in several crop species have been also associated with heat stress during seed development (Banowetz et al., 1999). For example, high temperature during wheat reproductive development hastens the decline in photosynthesis and leaf area, decreases shoot and grain mass as well as weight and sugar content of kernels, and also reduces water-use efficiency (Shah and Paulsen, 2003). As a consequence, heat stress results in an altered nutritional flour quality (Hedhly et al., 2009).

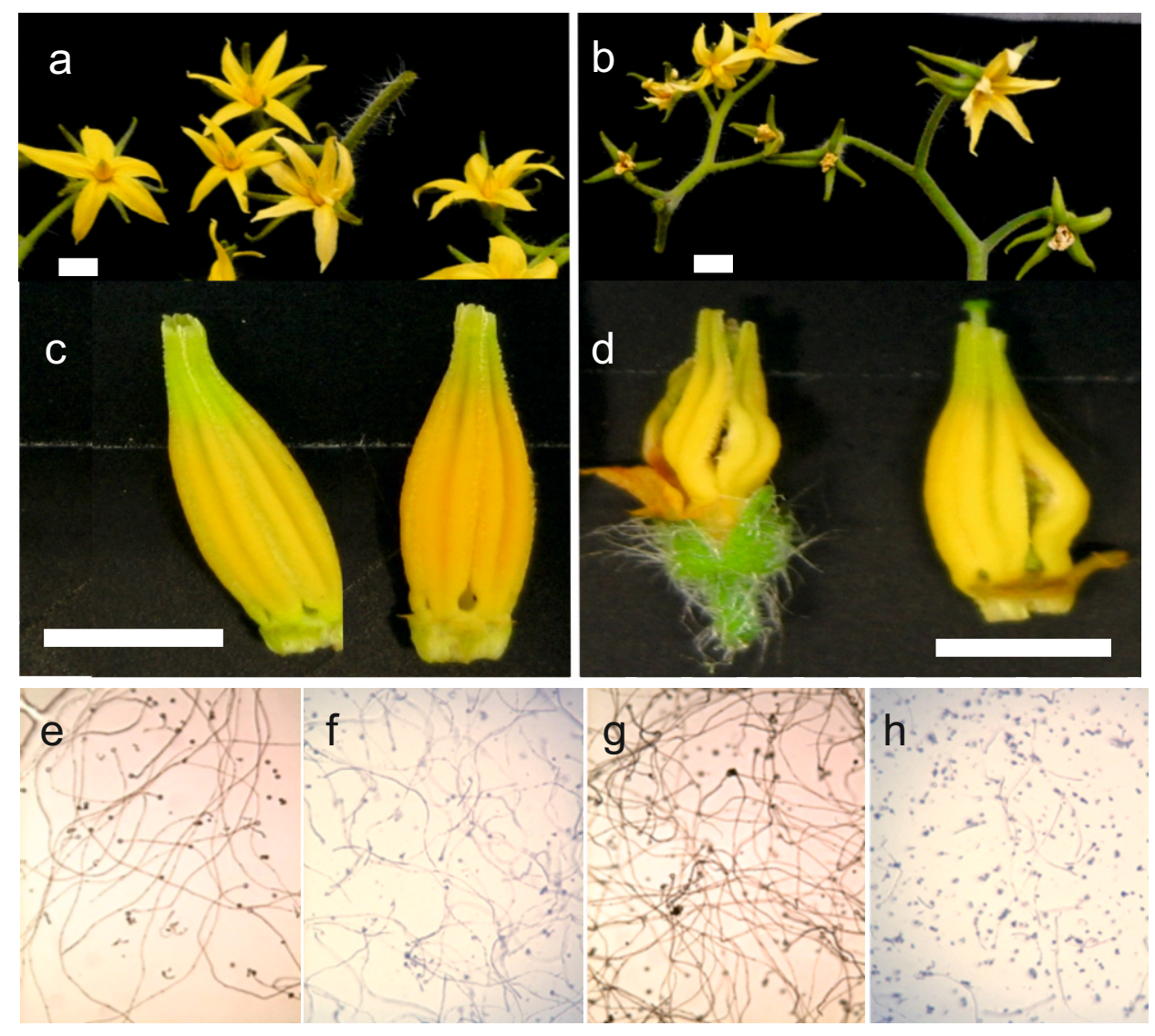

Figure 3. Flower development and pollen viability of tolerant (left) and sensitive tomato genotypes (right). The top shows inflorescences of a heat-tolerant genotype on the left (a) and a sensitive genotype on the right (b). In the second panel, the morphology of the anther cones is shown for both genotypes (c) and (d). Below (e-h) in vitro pollen germination under standard conditions ( $\mathrm{e}$; heat tolerant-genotype, g; heat sensitive) and high temperature for both genotypes (f; heat-tolerant genotype, h; heat sensitive).

In summary, high temperatures negatively affect various physiological processes including photosynthesis, primary and secondary metabolism, or lipid and hormonal signalling. Heat stress has negative effects on plant growth and development by disrupting the stability of various proteins, membranes and cytoskeleton structures. The most affected stage is the reproductive growth and the affected process is pollen grain development. Heat induces accumulation of heat shock proteins which prevent protein degradation and it also causes a state of metabolic imbalance and the build-up of toxic 
by-products, such as reactive oxygen species (ROS), which ultimately affect plant vegetative and reproductive development, with negative consequences on fruit-set and yield quality.

\section{Physiological and molecular indicators of tolerance to increased temperatures}

Plants possess various mechanisms to ensure survival under elevated temperatures. These strategies are classified into long-term phenological and morphological evolutionary adaptations such as changing leaf orientation, transpirational cooling or alterations in the membrane lipid composition, or short-term stress avoidance and acclimation mechanisms. At a molecular level, stress proteins, osmoprotectants, free-radical scavengers, ion transporters or factors involved in signalling cascades and transcriptional control are essential to counteract stress effects (Wang et al., 2004). Producing an economically significant yield under heat stress conditions depends on several plant physiological parameters and mechanisms that contribute to heat tolerance in the field, such as amendments to essential processes such as photosynthesis and concomitant increases of transcripts coding for proteins involved in protection. In many cases, a heat-tolerant variety is characterized by higher photosynthetic rates, increased membrane thermostability and heat avoidance (Nagarajan et al., 2010; Scafaro et al., 2010).

In all plant species, the ability to sustain leaf gas exchange under heat stress is directly correlated with heat tolerance. The decrease in the amount of active Rubisco can account for a large proportion of the negative temperature response of net photosynthesis (Salvucci and Crafts-Brandner, 2004) as the primary target site of thermal injury is carbon fixation by Rubisco. The reduction in carbon fixation and the consequent oxygen evolution result in generation of harmful reactive oxygen species. As a consequence, the repair mechanism of the damaged photo-system is inhibited. Hence, approaches to develop crops with improved productivity in a high temperature environment could include manipulating leaf photosynthesis and even photosynthate partitioning (Ainsworth and Ort, 2010).

One of the typical heat stress symptoms is tissue senescence, characterized by membrane damage associated with increased fluidity of membrane lipids, lipid peroxidation and protein degradation in various metabolic processes (Savchenko et al., 2002). Membrane lipid saturation is therefore considered an important element in high temperature tolerance. A higher share of saturated fatty acids in membrane lipids increases the lipid melting temperature and prevents a heat-induced increase in the membrane fluidity. Therefore, to maintain membrane fluidity, plants increase the content of saturated and monounsaturated fatty acids, modulating their metabolism in response to increasing temperatures (Zhang et al., 2005b). Thus, increasing the saturation level of fatty acids appears to be critical for maintaining membrane stability and enhancing heat tolerance (Larkindale and Huang, 2004).

Accumulation of osmo-protectants is an important adaptive mechanism in plants subjected to extreme temperatures, as primary metabolites participate directly in the osmotic adjustment (Sakamoto and Murata, 2000). For instance, accumulation of proline, glycine-betaine and soluble sugars is necessary to regulate osmotic activities and protect cellular structures from increased temperatures by maintaining the cell water balance, membrane stability and by buffering the cellular redox potential (Farooq et al., 2008). Transgenic approaches have confirmed the beneficial effect of proline overproduction during stress, as an enhanced proline production in transformed plants correlates well with a more negative leaf osmotic potential and higher production of protective xanthophyllic pigments under heat (Dobra et al., 2010).

Glycine betaine plays an important role as compatible solute in plants experiencing high temperature conditions (Sakamoto and Murata, 2002). Glycine betaine production in chloroplasts 
maintains the activation of Rubisco by sequestering Rubisco activase near thylakoids and preventing its thermal inactivation (Allakhverdiev et al., 2008). For example, high levels of glycine betaine accumulation were reported in maize and sugarcane in response to high temperature, while in contrast, plant species such as rice, mustard, Arabidopsis and tobacco naturally do not produce glycine betaine under stress conditions (Quan et al., 2004; Wahid and Close, 2007).

Studies suggest that high carbohydrate availability (e.g. glucose and sucrose) during heat stress represents an important physiological trait associated with heat-stress tolerance (Liu and Huang, 2000). Sucrose is the principal end product of photosynthesis, which translocates from source leaves to sink organs through the phloem. Sucrose and its cleavage products regulate plant development and response to stresses through carbon allocation and sugar signalling (Roitsch and González, 2004). Studies on an a heat-tolerant tomato genotype demonstrated that it is the high cell wall and vacuolar invertases activities and increased sucrose import into young tomato fruit that contribute to heat tolerance through increasing sink strength and sugar signalling activities (Li et al., 2012). Similarly, the carbohydrate content of developing and mature pollen grains may be an important factor in determining pollen quality, as heat-tolerant tomato cultivars appear to have a mechanism for maintaining the appropriate carbohydrate content under heat stress (Firon et al., 2006). In addition, sugars have been shown to also act as antioxidants in plants (Lang-Mladek et al., 2010). At low concentrations sucrose acts as signalling molecule while it has been suggested that in high concentrations it becomes a ROS scavenger (Sugio et al., 2009).

Enhanced synthesis of secondary metabolites under heat stress conditions also protects against oxidative damage. Several studies in tomato and watermelon indicate that thermal stress induces the accumulation of phenolics in the plant by activating their biosynthesis as well as inhibiting their oxidation, which could be an acclimation mechanism of the plant against thermal stress (Rivero et al., 2001). It has been suggested that in addition to their role as UV screen, anthocyanin accumulation under heat stress serve to decrease leaf osmotic potential, resulting in an increased uptake and reduced transpirational loss of water. These properties may enable the leaves to respond quickly to changing environmental conditions (Wahid et al., 2007).

Carotenoids protect various plant species from several stresses. For example, xanthophylls and some other terpenoids such as isoprene or tocopherol stabilize and photo-protect the lipid phase of the thylakoid membranes (Camejo et al., 2006; Velikova et al., 2005). Similarly, Arabidopsis plants overexpressing the chyB gene that encodes b-carotene hydroxylase (an enzyme active in the zeaxanthin biosynthetic pathway) show a greater tolerance to increased temperatures, and it was suggested that the protection from stress is most likely due to the action of zeaxanthin in preventing oxidative damage to membranes (Meiri et al., 2010).

Several lines of evidence indicate that separate from HSP induction, other pathways are involved in acquiring tolerance to heat. Several plant growth regulators, such as ABA, SA, ET, CK and AUX are proposed to play an important role in plant thermotolerance (Kotak et al., 2007). Under field conditions, ABA induction is an important component of thermotolerance due to its implication in survival under heat and desiccation stress (Maestri et al., 2002) and it is generally accepted that the ability to synthesise ABA under heat stress is the key factor attributed to the higher heat tolerance of plant cells (Ding et al., 2010). It has also been noted that the plant hormone ABA induces thermotolerance in maize (Musatenko et al., 2003). Pretreatment with SA increases basal tolerance to heat in an Arabidopsis mutant defective in SA signalling. In addition, transgenic plants unable to accumulate SA show up to $40 \%$ reduction in tolerance to heat (Clarke et al., 2004; Kaya et al., 2001). The maintenance of high levels of CKs in the kernels during heat stress appears to be important in increasing thermotolerance and providing yield stability in crop systems, as it is known that CKs have the potential to reduce oxidative stress in plants (Hare et al., 1997; Hsu et al., 2010). Foliar application of seaweed extract-based CK has been shown to increase leaf CK content and delay 
senescence of Agrostis sp. under heat and drought stress (Zhang and Ervin, 2008). In barley as well as in Arabidopsis, heat stress represses AUX signalling in an anther specific manner, leading to abortion of pollen development. As applying exogenous AUX entirely restores pollen development in heat conditions, it has been postulated that AUX promotes fertility under heat stress (Jaggard et al., 2010). In tomato and Arabidopsis, brassinosteroids also confer tolerance to high temperature stress by inducing the biosynthesis of major heat shock proteins (Bajguz and Hayat, 2009; Ogweno et al., 2008). Similarly, epibrassinolide treatment modulates the translational machinery, resulting in higher HSPs synthesis and rapid resumption of protein synthesis during and following the application of high temperature stress in Arabidopsis and rapeseed (Kagale et al., 2007).

\section{Molecular aspects of heat tolerance}

Plants are capable of adapting to a wide range of temperatures by reprogramming their transcriptome, proteome and metabolome and even by activating cell death mechanisms leading to organ abortion or entire plant death (Qi et al., 2011; Sánchez-Rodríguez et al., 2011). The ability to withstand or to acclimate to higher than optimal temperatures results from repair of heat-sensitive components and prevention of further heat injury, metabolic homeostasis being also maintained during stress. The most important characteristic of thermotolerance is the massive production of heat shock proteins (Vierling, 1991), however, as heat tolerance is a multigenic character, numerous biochemical and metabolic traits are also involved in the development and maintenance of thermotolerance: antioxidant activity, membrane lipid unsaturation, gene expression and translation, protein stability and accumulation of compatible solutes (Kaya et al., 2001). Nevertheless, plant responses to high temperatures clearly depend on genotypic parameters, as certain genotypes are more tolerant (Challinor et al., 2007; Prasad et al., 2006).

Several studies revealed that while some HSFs are critical for thermotolerance, others play a less critical role (HSP101, HSA32, HSFA1, HSFA3), since knockout variants of these proved to have little impact on tolerance to heat (Larkindale and Vierling, 2008; Schramm et al., 2008; Yoshida et al., 2011). These results also indicate that a complex regulatory network delivers a differential protection from heat stress. A recent study shows that in the absence of the HSFA1 transcription factor, a minimal yet significant level of acquired thermotolerance can still be attained in Arabidopsis mutants following acclimation, likely due to the induction of a small number of genes regulated by other transcription factors such as bZIP28 (Liu and Charng, 2012). Nevertheless, HSPs are of particular importance in thermotolerance reactions and act as molecular chaperones to prevent denaturation or aggregation of target proteins as well as facilitating protein refolding (Ahuja et al., 2010; Lohar and Peat, 1998; Scharf et al., 2011)

In many plant species, thermotolerance of cells and tissues after a heat stress is pretty much dependent upon induction of HSP70, though HSP101 has also been shown to be essential(Gurley, 2000). The enhanced expression of HSP70 was reported to assist in translocation, proteolysis, translation, folding, aggregation and refolding of denatured proteins (Gorantla et al., 2007; Zhang et al., 2010), while a methionine-rich chloroplast heat-shock protein has been shown to protect the thermolabile photosystem II and, consequently the whole-chain electron transport during heat stress (Peet et al., 2002). HSP101 for example, appears not to be required for growth in normal conditions, but plays a major role in tolerance to severe heat stresses and protein oxidative protection in Arabidopsis (Queitsch et al., 2000). Recently, it was however shown that an Arabidopsis mitochondrial transcription termination-factor related protein enhances thermotolerance in the absence of HSP101, via mitochondrial oxidative damage control (Kim et al., 2012). Several studies even support the hypothesis that HSFs can function as molecular sensors that are able to directly sense 
ROS, for instance $\mathrm{H}_{2} \mathrm{O}_{2}$, and control the expression of oxidative stress response genes during oxidative stress in Arabidopsis and tomato (Miller and Mittler, 2006; Volkov et al., 2006). Nuclear encoded sHSPs

Of the HSP gene family, induction of small HSP gene expression and protein accumulation upon environmental stresses point to the hypothesis that these proteins play an important role in stress tolerance (Sun et al., 2002). For example, some sHSPs become membrane associated, forming heat shock lipids that can stabilize membranes early on during the thermal stress. Introduction of the sHSP17.7 gene from carrot to potato was shown to enhance thermotolerance by affecting cellular membrane stability (Hu et al., 2010).

Recent studies also implicate the chloroplast protein synthesis elongation factor (EF-Tu) in plant response to high temperature stress. EF-Tu displays chaperone activity (Fu et al., 2008), and it has been suggested that high temperature-induced accumulation of EF-Tu is of importance in plant tolerance to high temperature stress (Fu et al., 2008; Prasad et al., 2008), as cultivars expressing a higher EF-Tu under high temperature stress were more tolerant to high temperature stress (Pressman et al., 2002).

In vitro experiments from several laboratories suggest that thermal stress at relevantly high temperatures produces ROS such as superoxide radicals, hydroxyl radicals and hydrogen peroxide at the chloroplastic PS II reaction centre, which are scavenged by antioxidants, including superoxide dismutase (SOD) (Bukhov and Mohanty, 1999). Tolerant plants generally protect themselves from the damaging effects of ROS with the synthesis of various antioxidant components, which control gene expression and influence essential processes such as growth, programmed cell death, abiotic stress responses and pathogen defence (Abiko et al., 2005). These components have been found in almost all cellular compartments, indicating the importance of ROS detoxification for cellular survival (Asada, 2006; Iba, 2002; Mittler et al., 2004). Essential for ROS detoxification particularly during stress are antioxidants such as ascorbic acid or glutathione and ROS-scavenging enzymes such as superoxide dismutase (SOD), ascorbate peroxidase (APX), catalase (CAT) or glutathione peroxidase (GPX).

In Arabidopsis for example, the APX gene family expression is heat stress dependent and regulated by HSF, evidence that links the heat stress response with oxidative stress and stress tolerance (Panchuk et al., 2002). Recently it was reported though that not all family members are positive regulators of tolerance to heat, as Arabidopsis plants deficient in an APX gene show enhanced seed production under extended heat stress conditions (Suzuki et al., 2013). Amelioration of oxidative damage to membranes could also represent another mechanism for maintaining membrane stability under heat stress (Kotak et al., 2007) and it has been recommended that targeting detoxification pathways might be an appropriate approach to engineer plants with multiple stresstolerance traits (Ristic et al., 2009).

Real time measurements of cytosolic calcium levels during heat stress in Arabidopsis show transient elevations in response to recovery from heating. The magnitude of these calcium peaks is greater in thermotolerant plants, implying that these calcium signals might play a role in mediating the effects of thermotolerance. Thus, examination of $\mathrm{Ca}^{2+}$ flux may allow the selection of heat-resistant varieties. Moreover, identification of the key players may allow targeting of the heat shock response to particularly relevant tissues, such as reproductive structures (Mach, 2012).

In plants, the acetylcholine (Ach)-mediated system, composed of ACh, ACh receptor (AChR), and AChE plays a significant role in signal transduction, as it does in animals. Overexpression of maize catabolic gene AChE in transgenic tobacco plants enhances heat tolerance relative to that of non-transgenic plants, suggesting thus that AChE plays a positive role not only in maize but also in tobacco heat tolerance. Native tropical zone plants also show high AChE activity during heat stress. Therefore, engineering plant AChE might be useful in breeding for enhanced heat tolerance 
(Yamamoto et al., 2011). Heat induced expression of various proteases is also important in regulating plant responses to heat stress. For example, the FtsH11 protease of Arabidopsis has been found to contribute to overall tolerance to high temperatures by alleviating light stress through the degradation of unassembled thylakoid membrane proteins (Chen et al., 2006). HOT5, which encodes an alcohol dehydrogenase functioning as nitrosoglutathione reductase, is also required for survival under heat, revealing a possible role of nitric oxide (NO) in thermotolerance and plant development (Sivasankar et al., 2012).

Late embryogenesis abundant (LEA) proteins, ubiquitin, and dehydrins have been found to play important roles in protection from heat and drought stress. For example, LEA proteins can prevent aggregation and protect the citrate synthase (involved in ATP production) from desiccating conditions like heat and drought stress (Willits and Peet, 1998). Similarly, ubiquitin and conjugatedubiquitin synthesis during the first $30 \mathrm{~min}$ of exposure emerged as an important mechanism of heat tolerance in mesquite and soybean experiencing heat stress (Huang and $\mathrm{Xu}, 2008$ ).

With respect to tolerance responses of reproductive tissues, approaches to discover the molecular mechanisms conferring heat tolerance during pollen development are a valuable avenue help develop heat-tolerant germplasm. Genome-wide strategies are being used to investigate all aspects of pollen development, including responses to temperature stress (Bita et al., 2011; Frank et al., 2009; Jagadish et al., 2010b). Several studies reveal that HSPs are not the only players involved in response to heat in the reproductive structures, but other components are involved as well, such as hormones and antioxidants (Sakata et al., 2010).

Several genes have been identified in tolerant genotypes or wild germplasm, which were shown to improve photosynthesis and protect reproductive development, such as HSP101, stable Rubisco isoforms, etc. However, many genes identified from anther profiling under heat show no homology to known sequences and represent potential candidates for further study of the tolerance to heat (Zinn et al., 2010). An analysis of tomato maturing microspores revealed increased expression of SIAPX3 (a reactive oxygen species scavenger), ET responsive genes (including the MBF1 homologue ER24), HSFA2, and HSFA3, and HSP family members. In addition, a calcium dependent protein kinase 2 (CDPK2) was up-regulated in the heat stressed microspores, but whether the heat stress induction of this gene results in a more active stress tolerance response is still to be determined (Frank et al., 2009).

High temperature stress is detrimental to cereal crop productivity and the existence of genetic variability in heat stress tolerance is an indispensable factor for the development of more tolerant cultivars. For example, tolerant wheat genotypes are defined by maintenance of photosynthesis, chlorophyll content, and stomatal conductance under heat stress, while the yield of these genotypes is maintained through higher seed set, grain weight, and extended grain filling duration even at elevated temperatures (Yang et al., 2002). When there is a limited variability for tolerance to heat within a crop species, wild germplasm can also be used as tolerance source (Pradhan et al., 2012b).

In a heat-tolerant rice variety, multiple protein components of the Calvin Cycle increased in expression under heat stress, including a consistent increase of phosphoribulokinase (PRK), the enzyme responsible for the final step in Ribulose-1,5-bisphosphate (RuBP) regeneration. The protective proteins Cpn60, HSP90, and HSP70 also increased not only in protein abundance but also in gene expression and even a thiamine biosynthesis protein (THI1), previously shown to have a protective role against stress, increased its levels during the heat stress (Scafaro et al., 2010).

Similarly, a proteomic analysis comparing proteins expressed in heat-stressed anthers from three rice varieties with different temperature tolerances, revealed a higher accumulation of sHSPs in the most tolerant rice genotype, compared with the most sensitive rice genotype, while the moderately tolerant rice genotype showed intermediate sHSPs accumulation. It was therefore proposed that the accumulation of sHSPs might confer greater heat tolerance in rice (Jagadish et al., 2010b). 
Genetic variation in the ability of tomatoes to set fruit under high temperature conditions, has made selection for heat tolerance possible. For example, in several genotypes differing in their capacity for thermotolerance, as well as in sugarcane, an increased chlorophyll a:b ratio is observed in the tolerant genotypes under high temperatures, indicating that these changes are related to thermotolerance (Camejo et al., 2006; Wahid, 2007). A comparative anther transcriptome analysis between tolerant and sensitive tomato genotypes shows that the metabolism and development-related genes are more highly expressed in the heat-sensitive genotypes, while gene modulation intensity suggests that the difference in tolerance to heat stress is associated with a lower percentage of transcripts affected in the heat-tolerant genotypes; however, HSP induction remains higher in the tolerant genotypes (Bita et al., 2011; Frank et al., 2009). Several tolerant grape genotypes show higher levels of HSP70 and genes related to metabolism and stress protection under elevated temperatures and one tolerant genotype showed higher heat shock gene expression levels even under standard conditions (Zhang et al., 2005a).

White goosefoot populations native to more stressful habitats or grown at higher temperatures have lower HSP levels and induced thermotolerance, relying apparently on basal mechanisms for thermotolerance and it has been suggested that future global climate change will differentially impact ecotypes within species, possibly by selecting for increased basal versus inducible thermotolerance (Barua et al., 2008). Indeed, higher basal gene expression levels under non-challenged conditions have also been measured for microspores of a heat-tolerant tomato cultivar. The detected genes are considered as interesting candidates for microspore thermotolerance. (Frank et al., 2009). Hence, the inherent genetic variability for tolerance can be exploited to our advantage and genes that confer stress tolerance can be sourced from germplasm collections, wild relatives or organisms that live in extreme habitats.

Global warming will have significant impacts on future crop yields. In the view of the predicted population growth and the resulting increasing requirement for food security, it is up to the scientific community to adapt crop species for high tolerance to abiotic stresses and in particular high temperature stress. A more complete insight of the biological processes behind the heat stress response combined with classical and emerging technologies in plant breeding and genetic engineering is likely to make a significant contribution to improved crops.

\section{Strategies to crop improvement for heat stress tolerance}

Generally, tolerance to heat is characterized by a lesser effect on essential processes such as photosynthesis and by consistent increases of transcripts involved in the biosynthesis of protective components. As photosynthesis and reproductive development are the most sensitive physiological processes to stress (Prasad et al., 2008), a heat-tolerant variety will be usually characterized by higher photosynthetic rates reflected in stay-green leaves, increased membrane-thermostability and successful fruit-set under high temperature conditions (Nagarajan et al., 2010; Scafaro et al., 2010).

Multiple opportunities for plant improvement exist, as tolerance to high temperatures is a multigenic character. Screening for heat tolerance in the field presents a challenge due to interactions with other environmental factors but a wide variety of screenable traits is available that allows successful selection in the field (Hall, 2011). Tolerant genotypes may also be selected in controlled environments. However, besides being more expensive, controlled environments do not allow natural selection for other factors that interact with the heat-stress tolerance mechanisms under field conditions (Souza et al., 2012). Regardless the screening method, a key objective for plant breeders is to develop an effective set of thermotolerance markers which can be used to further implement heat tolerance into various crop species. 
Since plants adapt to temperature stress by developing more appropriate morphological, physiological and biochemical characteristics, analysing plant phenology in response to heat stress often gives a better understanding of the plant response and facilitates further molecular characterization of the tolerance traits (Wahid et al., 2007). The emerging phenomics methodologies are used to identify genes associated with traits of interest by establishing functional relationships between genetics and the associated phenotype. Such tools are also used to characterize plant performance under controlled environments or in the field (Sivasankar et al., 2012). The temperature and duration of heat stress treatments resulting in changes in growth and development vary between plant tissues and growth stages so choosing an appropriate phenotype is critical as the function of a heat stress response gene may contribute to thermotolerance differentially across tissues and growth stages. Ideally, systematic phenotyping approach that includes a range of heat stress conditions may increase the chances of identifying the functions of potential heat stress response genes.

Despite previous characterisation of genes that ameliorate the effects of high temperature, direct trait selection based on molecular markers remains difficult. Thus, a more productive alternative to genetic engineering would be selection for indirect physiological traits related to tolerance. For example, in many crop plants, early maturation under high temperatures is closely correlated with smaller yield losses (Adams et al., 2001). Furthermore, the genetic variability for tolerance to heat stress present in rice could be exploited to screen germplasm and select cultivars that open flowers earlier in the morning or that maintain a high number of spikelets/panicle when grown in warm environments (Singh et al., 2010). Similarly, in wheat, a positive correlation between canopy temperature depression and grain yield has been reported and recommended as a useful trait in selecting high temperature tolerant genotypes (Pradhan et al., 2012a).

At cellular level, electron transport rate, membrane integrity, and enzyme viability have also been successfully used to screen for heat tolerance under field conditions (Cottee et al., 2010). Chlorophyll accumulation assays have been used to characterise genetic variability in acquired thermotolerance for many crop species (Selvaraj et al., 2011). In wheat for instance, heat stress induced damage of the thylakoid membrane is closely associated to chlorophyll loss, and detection of chlorophyll content has been proposed as high throughput screening method for tolerance to heat (Shah and Paulsen, 2003).

Pollen viability and seed-set as measures of reproductive success under heat stress are also candidate traits for selection in breeding programs, and generally there is a strong correlation between pollen production and viability, anther dehiscence, and seed-set. The anthers of heat-tolerant rice cultivars dehisce more easily than those of susceptible cultivars under high temperature conditions (Jagadish et al., 2010a; Prasad et al., 2006).

Breeding for stress tolerance requires efficient screening procedures, identification of key traits in diverse donor or tolerant lines and understanding their inheritance and molecular genetics. Molecular genetic markers are an example of how an effective tool is used to analyse plant genomes and how heritable traits associate to their underlying genetic variation. Sequence-based (microarrays) or anonymous molecular marker systems (AFLP; (Vos et al., 1995) are often employed in applications of modern plant genetic analysis. Moreover, the reducing cost of DNA sequencing and increasing availability of large sequence data sets will allow mining for large numbers of such markers. Further on, the markers can be introduced in genetic linkage analyses and trait mapping, association studies and marker-assisted selection (Duran et al., 2010). At this time, sequencing technology is no longer the bottleneck for the generation of sequence data from whole genome shotgun sequencing (WGS) and thus, the generation of marker data has become a bioinformatics issue rather than a cost or time issue (Egan et al., 2012). For the major crops, including those that could deliver the largest impact on improvement of yield security under the expected higher global temperatures, re-sequencing will become the method of choice for identifying markers that co- 
segregate with temperature tolerance traits. The wealth of marker data generated by WGS, facilitates the implementation of these very high resolution marker datasets for quantitative locus (QTL) mapping and genome wide association studies (GWAS).

The search for molecular markers associated to phenotypic traits is one aspect of molecular genetics usually carried out with methods based on segregation mapping, genomic introgression, and association mapping (Morgante and Salamini, 2003). For example, quantitative trait loci (QTL) mapping has recently become the method of choice to identify specific chromosome segments that contain candidate genes for heat tolerance (Argyris et al., 2011; Zhang et al., 2012).

Quantitative trait locus (QTL) analysis in tolerant and sensitive crops is now receiving much attention. The key benefit of QTL-based approaches is that they allow loci to be identified that are linked to heat tolerance. The identification of markers linked to QTLs enables breeding of stresstolerant crops by combining or 'pyramiding' QTLs for tolerance to various stresses. Several QTL studies relating to various abiotic stress tolerances have already been reported (Hirayama and Shinozaki, 2010).

Detecting adaptive quantitative trait loci (QTLs) for high-temperature tolerance is one way to understand tolerance mechanisms and several studies have already identified genetic markers related to different environmental stresses, including heat (Roy et al., 2011). QTL mapping studies for heat tolerance have been conducted on various rice populations at flowering stages. However, confirmation and fine mapping of the identified QTLs for heat tolerance have not been reported yet (Ye et al., 2012). Multiple loci for heat tolerance have been identified in wheat (Paliwal et al., 2012) and maize (Bai, 2011). A study on Arabidopsis mutants sensitive to heat also revealed QTLs involved in acquiring thermotolerance (Hong et al., 2003). A major QTL for high temperature germination and an additional QTL having smaller effects were identified as well in a genetic analysis of lettuce seed thermo-inhibition (Argyris et al., 2008). The markers linked to these QTLs could be used to improve heat tolerance in available germplasm. At present, high temperature tolerance QTL identification is performed using different traits, such as the thousand-grain weight (TGW), the grain filling duration (GFD), canopy temperature depression (CTD), yield (Pinto et al., 2010) or senescence related traits (Vijayalakshmi et al., 2010). In order to transfer these traits, classical breeding requires the establishment of rapid and cost effective screening procedures and implementing these using conventional non-transgenic breeding approaches such as "marker assisted screening", association mapping or genomic selection procedures.

Recently, association genetics has started to assist in QTL identification in several crop species (Ahuja et al., 2010). Association mapping or linkage disequilibrium mapping is a highresolution and relatively low cost methodology, that might likely be used to identify traits associated with abiotic stresses, in combination with high-throughput marker genotyping platforms (Varshney et al., 2009). Once the markers associated with QTLs have been isolated, the candidate QTLs can be further introgressed in elite lines through marker-assisted selection strategies. One of the difficulties of developing superior genotypes for heat stress is that these traits are generally controlled by small effect QTLs or several epistatic QTLs. In order to overcome this problem, pyramiding several QTLs from large populations in the same genetic background, marker-assisted recurrent selection (MARS) or genomic selection (GS) approaches can be engaged (Tester and Langridge, 2010)

Marker-assisted selection (MAS) approaches have significantly contributed to revealing the genetic basis of plant stress tolerance in some crops and even led to the release of plants with enhanced tolerance to abiotic stress (Lopes and Reynolds, 2010; Thomson et al., 2010). However, MAS programs for complex traits such as tolerance to heat are not effective mainly due to the genotype $\times$ environment and gene-gene (i.e. epistasis) interactions, which frequently results in a low breeding efficiency (Collins et al., 2008). In contrast to MAS strategies which use markers for which a significant association with a trait has been identified, the genomic selection method predicts breeding 
values using data derived from a vast number of molecular markers with a high coverage of the genome. Its novelty is that it uses all marker data as predictors of performance and subsequently delivers more accurate predictions. Simulation studies indicated that genomic selection may increase the correlations between predicted and true breeding-value over several generations, without the need to re-phenotype. Thus, genomic selection may result in lower analysis costs and increased rates of genetic gain (Habier et al., 2009; Heffner et al., 2009).

However, QTLs often do not translate well across genetic backgrounds and often produce smaller than expected adaptation effects. To improve crop abiotic stress tolerance by exploiting the segregation of natural alleles seems thus rather challenging for such an adaptive QTL strategy (Collins et al., 2008). When quantitative hereditary characteristics such as heat stress tolerance are involved, recurrent selection seems to be one of the most efficient methods in plant breeding. In multiple crosses, the probability is very small of obtaining superior genotypes that reunite all the favourable alleles. However, in this circumstance, a large segregating population is required, aspect that becomes unfeasible in practice.

The alternative is to adopt recurrent selection to gradually accumulate, by recombination cycles, the desirable and available alleles in different parents (Doná et al., 2012). The primary purpose of a recurrent selection program is to increase the frequency of favourable alleles for traits of interest, conserving the genetic variability. Advantages are a greater genetic variability obtained by inter-crossing of multiple parents; (b) greater opportunity for recombinations because of successive crossings; (c) greater efficiency in increasing the favourable gene frequency because the process is repetitive and accumulative; (d) greater facility to incorporate exotic germplasm in the population (Ramalho et al., 2005). For example, the potato-breeding program at the Federal University of Lavras has also been successful in developing heat-stress tolerance genotypes using recurrent selection obtaining expressive gains in tuber production with improved quality (Benites and Pinto, 2011).

Obviously, only field trials under real stress conditions allow for conclusive remarks on stress tolerance and yield performance of a genotype (Mason et al., 2010). However, the lack of a precise phenotyping protocol is most probably the limiting factor in the genetic analysis of quantitative traits. Development of more precise phenotyping tools that can be applied to field conditions is a prerequisite for enabling the assessment of the complex genetic networks associated with QTLs. The small yet significant phenotypic changes delivered by introducing single genes into breeding material require precision phenotyping protocols and the capacity to carry these out on very large populations (Cattivelli et al., 2008).

To transfer advantageous traits to a cultivar, modern (genetic modification) approaches can also be employed. The transgenic approach requires identification of the gene responsible for the desired trait but poses no barrier to transferring useful genes across different species within the plant kingdom or even from animal systems. Genes of non-plant species could potentially be introgressed as well and generally, several combinations of beneficial genes could be transferred into the same plant. Transformation protocols are available for most important food crop species. However, the regulatory restrictions for the use of transgenic plants make this technology economically unpractical. Moreover, the existing methods have a diminished efficiency for cereal crops such as wheat and barley and even rice (Takeda and Matsuoka, 2008).

Nevertheless, to combine stress tolerance with high yield potential while avoiding the negative effects of a stress gene on plant growth under favourable conditions, strategies that spatially and temporally restrict transgene expression via tissue specific and stress-inducible promoters are used (Nakashima et al., 2007). Engineering promoters will facilitate gene pyramiding through genetic modification, addressing the issue of tolerance to multiple stresses at different stages of plant growth (Datta et al., 2002). As an alternative, engineering with specific transcription factors and signalling 
components could be employed. Ultimately that leads to the expression of their target transcriptome that consists of several genes involved in the response to stress.

Transcriptome engineering emerges thus as a promising avenue for the development of abiotic stress-tolerant crops. Currently however, plant genetic engineering is hampered by nonbiological constraints mainly related to the commercialisation of transgenic crops, particularly in Europe (Cattivelli et al., 2008). Thus, the future commercial success of transgenic breeding will depend upon the development of clearly defined and scientifically based regulatory frameworks, and upon public acceptance of genetically modified plants and their produce (Godfray et al., 2010).

\section{Conclusions}

It is now well accepted that the complexity of the heat syndrome can only be tackled with a holistic approach that integrates examination of crop heat tolerance traits by classical and modern molecular genetic tools with agronomic practices resulting thus in superior crop genotypes. The polygenic basis of heat tolerance and the issues of detecting minor QTLs with molecular markers strongly limit the use of MAS to identify heat tolerance related traits by classical genetics. High throughput sequencing and the predicted decline in genotyping costs will assist in obtaining a denser genome wide marker coverage for all crop species, accelerate QTL discovery and transfer by genomic selection and confer an increased flexibility to the interplay between phenotypic evaluation and selection (Heffner et al., 2009).

Heat stress tolerance is a polygenic character often measured using complex traits such as yield under stress, which implicate many processes and mechanisms. Therefore, introgression of a gene or QTL by conventional or modern breeding is usually not sufficient to develop heat-tolerant lines, unless there is a large effect on a particular key process. In addition, achieving highest genetic gain via classical methods requires a just choice for the appropriate breeding strategy (i.e. recurrent or genomic selection).

The manipulation of major regulatory genes through biotechnology is considered to be more efficient than conventional breeding through serial hybridizations. Linking several beneficial genes (transgenic pyramiding) into a commercial variety via the transgenic approach is also likely to provide a key route to crop improvement. A likely drawback is that the function of homologous genes is often diverged between species. Thus, for practical reasons, marker-assisted backcrossing of a transgene into a commercially viable variety will be necessary. In addition, to avoid negative effects on plant growth, it will be necessary to restrict transgene expression to a specific tissue or stress, via the use of tissue specific and stress-inducible promoters. As yet, a limited number of reports have been published on QTL validation or introgression and the field-testing of transgenic plants (Varshney et al., 2011).

Until now, very few tolerant varieties have been released, mainly due to regulatory issues associated with GMOs or due to the difficult and lengthy process of breeding for tolerance. There is also a great concern that laboratory testing does not reflect true field conditions. In many laboratory studies on stress tolerance of genetically modified plants, tolerance is tested either against a single stress or is examined over relatively short periods of time. In contrast, in field conditions, plants are simultaneously subjected to various stresses and in some circumstances these constraints extend throughout their lifetimes. As consequence, to identify and understand these interactions it will be necessary to perform thermotolerance phenotyping with multiple stresses. Abiotic stresses affect plants in a complex manner depending on the growth stage. The conventional and molecular plant breeding efforts are limited by the present lack of accurate phenotyping for stress responses, due to the incomplete understanding of tolerance mechanisms. For example, developing countries are still 
lacking in appropriate phenotyping facilities in the public research sector. In addition, generating high yielding and stress tolerant crops requires a thorough understanding of the metabolic and developmental processes involved not only in stress responses but also in energy regulation (Hirayama and Shinozaki, 2010). For instance, combining different approaches led to the development of rice cultivars referred to as "Green Super Rice", tolerant to several stresses and having a high nutritious value, promising to greatly reduce the consumption of pesticides, chemical fertilizers or water (Yang and Zhang, 2010). Such strategies are likely to improve stress tolerance but nonetheless, there is a lag from basic research to the production of stress-tolerant crops.

Significant emphasis has been put on molecular biology as the newest agricultural research tool to detect molecular markers associated with stress related traits, as well as individual candidate genes for stress tolerance (Barnabás et al., 2008). Nevertheless, its value in providing short-term solutions for heat stress tolerance is unknown due to the complexity of the genetic background for desirable physiological traits and their interaction with the environment (Savin and Slafer, 2010). The use of mathematical modelling in conjunction with genetic information is emerging as an additional methodology to assist the identification of physiological traits for new plant ideotypes (Semenov and Halford, 2009). Ultimately, differences in their primary concerns and research strategies cause molecular biologists, plant physiologists and breeders to work independently, where an intimate collaboration amongst them is required. Such integration will enable identification of tolerance genes and elucidate the functional relationships between genotype and observed phenotypes, providing thus a system-wide phenome to genome analysis, while enabling accurate trait mapping, introgression of superior alleles or cloning of major QTLs for complex characters such as abiotic stress tolerance. The transgenic approach must be combined with the efforts in marker-assisted breeding programs for stress related genes and QTLs for obtaining effective high temperature tolerance.

Particularly in the view of the predicted global warming, understanding the molecular basis of the relevant agronomic traits is essential to allow breeders to design new ideotypes in silico, construct new genotypes in planta and ultimately implement these across a wide range of environmental conditions and locations. 


\section{References}

Abiko, M., Akibayashi, K., Sakata, T., Kimura, M., Kihara, M., Itoh, K., Asamizu, E., Sato, S., Takahashi, H. and Higashitani, A. (2005) High-temperature induction of male sterility during barley (Hordeum vulgare L.) anther development is mediated by transcriptional inhibition. Sexual Plant Reproduction 18, 91-100.

Adams, S., Cockshull, K. and Cave, C. (2001) Effect of temperature on the growth and development of tomato fruits. Annals of botany 88, 869-877.

Ahmad, A., Diwan, H. and Abrol, Y.P. (2010) Global climate change, stress and plant productivity. Abiotic Stress Adaptation in Plants, 503-521.

Ahuja, I., de Vos, R.C.H., Bones, A.M. and Hall, R.D. (2010) Plant molecular stress responses face climate change. Trends in plant science 15, 664-674.

Ainsworth, E.A., Beier, C., Calfapietra, C., Ceulemans, R., Durand-Tardif, M., Farquhar, G.D., Godbold, D.L., Hendrey, G.R., Hickler, T., Kaduk, J., Karnosky, D.F., Kimball, B.A., Korner, C., Koornneef, M., Lafarge, T., Leakey, A.D., Lewin, K.F., Long, S.P., Manderscheid, R., McNeil, D.L., Mies, T.A., Miglietta, F., Morgan, J.A., Nagy, J., Norby, R.J., Norton, R.M., Percy, K.E., Rogers, A., Soussana, J.F., Stitt, M., Weigel, H.J. and White, J.W. (2008) Next generation of elevated [CO2] experiments with crops: a critical investment for feeding the future world. Plant, cell \& environment 31, 1317-1324.

Ainsworth, E.A. and Ort, D.R. (2010) How do we improve crop production in a warming world? Plant physiology 154, 526-530.

Akman, Z. (2009) Comparison of high temperature tolerance in maize, rice and sorghum seeds by plant growth regulators. Journal of Animal and Veterinary Advances 8, 358-361.

Allakhverdiev, S.I., Kreslavski, V.D., Klimov, V.V., Los, D.A., Carpentier, R. and Mohanty, P. (2008) Heat stress: an overview of molecular responses in photosynthesis. Photosynthesis research 98, 541-550.

Argyris, J., Dahal, P., Hayashi, E., Still, D.W. and Bradford, K.J. (2008) Genetic variation for lettuce seed thermoinhibition is associated with temperature-sensitive expression of abscisic acid, gibberellin, and ethylene biosynthesis, metabolism, and response genes. Plant physiology 148, 926-947.

Argyris, J., Truco, M.J., Ochoa, O., McHale, L., Dahal, P., Van Deynze, A., Michelmore, R.W. and Bradford, K.J. (2011) A gene encoding an abscisic acid biosynthetic enzyme (LsNCED4) collocates with the high temperature germination locus Htg6. 1 in lettuce (Lactuca sp.). TAG Theoretical and Applied Genetics 122, 95-108.

Asada, K. (2006) Production and scavenging of reactive oxygen species in chloroplasts and their functions. Plant physiology 141, 391-396.

Bai, J. (2011) Genetic variation of heat tolerance and correlation with other agronomic traits in a maize (Zea mays L.) recombinant inbred line population.

Bajguz, A. and Hayat, S. (2009) Effects of brassinosteroids on the plant responses to environmental stresses. Plant Physiology and Biochemistry 47, 1-8.

Banowetz, G., Ammar, K. and Chen, D. (1999) Temperature effects on cytokinin accumulation and kernel mass in a dwarf wheat. Annals of botany 83, 303-307.

Banti, V., Mafessoni, F., Loreti, E., Alpi, A. and Perata, P. (2010) The heat-inducible transcription factor HsfA2 enhances anoxia tolerance in Arabidopsis. Plant physiology 152, 1471-1483.

Barnabás, B., Jäger, K. and Fehér, A. (2008) The effect of drought and heat stress on reproductive processes in cereals. Plant, cell \& environment 31, 11-38.

Barua, D., Heckathorn, S.A. and Coleman, J.S. (2008) Variation in Heat-shock Proteins and Photosynthetic Thermotolerance among Natural Populations of Chenopodium album L. from Contrasting Thermal Environments: Implications for Plant Responses to Global Warming. Journal of integrative plant biology 50, 1440-1451.

Battisti, D.S. and Naylor, R.L. (2009) Historical warnings of future food insecurity with unprecedented seasonal heat. Science 323, 240-244.

Benites, F.R.G. and Pinto, C.A.B.P. (2011) Genetic gains for heat tolerance in potato in three cycles of recurrent selection. Crop Breeding and Applied Biotechnology 11, 133-140. 
Binder, B.M. and Patterson, S.E. (2009) Ethylene-dependent and-independent regulation of abscission. Stewart Postharvest Review 5, 1-10.

Bita, C.E., Zenoni, S., Vriezen, W.H., Mariani, C., Pezzotti, M. and Gerats, T. (2011) Temperature stress differentially modulates transcription in meiotic anthers of heat-tolerant and heatsensitive tomato plants. BMC genomics 12, 384 .

Bukhov, N. and Mohanty, P. (1999) Elevated temperature stress effects on photosystems: characterization and evaluation of the nature of heat induced impairments. Singhal GS, Renger G, Sopory SK, Irrgang KD and Govindjee (eds) Concepts in Photobiology: Photosynthesis and Photomorphogenesis, 617-648.

Camejo, D., Jiménez, A., Alarcón, J.J., Torres, W., Gómez, J.M. and Sevilla, F. (2006) Changes in photosynthetic parameters and antioxidant activities following heat-shock treatment in tomato plants. Functional plant biology 33, 177-187.

Cattivelli, L., Rizza, F., Badeck, F.W., Mazzucotelli, E., Mastrangelo, A.M., Francia, E., Mare, C., Tondelli, A. and Stanca, A.M. (2008) Drought tolerance improvement in crop plants: An integrated view from breeding to genomics. Field Crops Research 105, 1-14.

Challinor, A., Wheeler, T., Craufurd, P., Ferro, C. and Stephenson, D. (2007) Adaptation of crops to climate change through genotypic responses to mean and extreme temperatures. Agriculture, ecosystems \& environment 119, 190-204.

Chen, J., Burke, J.J., Velten, J. and Xin, Z. (2006) FtsH11 protease plays a critical role in Arabidopsis thermotolerance. The Plant journal : for cell and molecular biology 48, 73-84.

Chen, J., Wang, P., Mi, H.-1., Chen, G.-Y. and Xu, D.-Q. (2010) Reversible association of ribulose-1, 5-bisphosphate carboxylase/oxygenase activase with the thylakoid membrane depends upon the ATP level and $\mathrm{pH}$ in rice without heat stress. Journal of experimental botany 61, 29392950.

Chhetri, N. and Chaudhary, P. (2011) Green Revolution: Pathways to Food Security in an Era of Climate Variability and Change? Journal ref: Journal of Disaster Research 6, 486-497.

Christensen, J.H. and Christensen, O.B. (2007) A summary of the PRUDENCE model projections of changes in European climate by the end of this century. Climatic Change 81, 7-30.

Clarke, S.M., Mur, L.A., Wood, J.E. and Scott, I.M. (2004) Salicylic acid dependent signaling promotes basal thermotolerance but is not essential for acquired thermotolerance in Arabidopsis thaliana. The Plant journal : for cell and molecular biology 38, 432-447.

Collins, N.C., Tardieu, F. and Tuberosa, R. (2008) Quantitative trait loci and crop performance under abiotic stress: Where do we stand? Plant physiology 147, 469-486.

Cottee, N., Tan, D., Bange, M., Cothren, J. and Campbell, L. (2010) Multi-Level Determination of Heat Tolerance in Cotton (L.) under Field Conditions. Crop Sci 50, 2553-2564.

Datta, K., Baisakh, N., Maung Thet, K., Tu, J. and Datta, S. (2002) Pyramiding transgenes for multiple resistance in rice against bacterial blight, yellow stem borer and sheath blight. TAG Theoretical and Applied Genetics 106, 1-8.

Ding, W., Song, L., Wang, X. and Bi, Y. (2010) Effect of abscisic acid on heat stress tolerance in the calli from two ecotypes of Phragmites communis. Biologia plantarum 54, 607-613.

Dobra, J., Motyka, V., Dobrev, P., Malbeck, J., Prasil, I.T., Haisel, D., Gaudinova, A., Havlova, M., Gubis, J. and Vankova, R. (2010) Comparison of hormonal responses to heat, drought and combined stress in tobacco plants with elevated proline content. Journal of plant physiology 167, 1360-1370.

Doná, A.A., Miranda, G.V., DeLima, R.O., Chaves, L.G. and e Gama, E.E.G. (2012) GENETIC PARAMETERS AND PREDICTIVE GENETIC GAIN IN MAIZE WITH MODIFIED RECURRENT SELECTION METHOD. CHILEAN JOURNAL OF AGRICULTURAL RESEARCH 72, 1.

Duran, C., Eales, D., Marshall, D., Imelfort, M., Stiller, J., Berkman, P.J., Clark, T., McKenzie, M., Appleby, N. and Batley, J. (2010) Future tools for association mapping in crop plants. Genome / National Research Council Canada = Genome / Conseil national de recherches Canada 53, 1017-1023.

Easterling, W. and Apps, M. (2005) Assessing the consequences of climate change for food and forest resources: a view from the IPCC. Increasing Climate Variability and Change, 165-189. 
Egan, A.N., Schlueter, J. and Spooner, D.M. (2012) Applications of next-generation sequencing in plant biology. American Journal of Botany 99, 175-185.

Ellis, R.J. (1990) Molecular chaperones: the plant connection. Science 250, 954-959.

Endo, M., Tsuchiya, T., Hamada, K., Kawamura, S., Yano, K., Ohshima, M., Higashitani, A., Watanabe, M. and Kawagishi-Kobayashi, M. (2009) High temperatures cause male sterility in rice plants with transcriptional alterations during pollen development. Plant and cell physiology 50, 1911-1922.

Farooq, M., Basra, S., Wahid, A., Cheema, Z., Cheema, M. and Khaliq, A. (2008) Physiological role of exogenously applied glycinebetaine to improve drought tolerance in fine grain aromatic rice (Oryza sativa L.). Journal of Agronomy and Crop Science 194, 325-333.

Firon, N., Shaked, R., Peet, M., Pharr, D., Zamski, E., Rosenfeld, K., Althan, L. and Pressman, E. (2006) Pollen grains of heat tolerant tomato cultivars retain higher carbohydrate concentration under heat stress conditions. Scientia Horticulturae 109, 212-217.

Fischer, R. and Edmeades, G.O. (2010) Breeding and cereal yield progress. Crop Sci 50, S-85-S-98.

Foyer, C.H. and Noctor, G. (2009) Redox regulation in photosynthetic organisms: signaling, acclimation, and practical implications. Antioxidants \& redox signaling 11, 861-905.

Frank, G., Pressman, E., Ophir, R., Althan, L., Shaked, R., Freedman, M., Shen, S. and Firon, N. (2009) Transcriptional profiling of maturing tomato (Solanum lycopersicum L.) microspores reveals the involvement of heat shock proteins, ROS scavengers, hormones, and sugars in the heat stress response. Journal of experimental botany 60, 3891-3908.

Fu, J., Momcilovic, I., Clemente, T.E., Nersesian, N., Trick, H.N. and Ristic, Z. (2008) Heterologous expression of a plastid EF-Tu reduces protein thermal aggregation and enhances $\mathrm{CO} 2$ fixation in wheat (Triticum aestivum) following heat stress. Plant molecular biology 68, $277-288$.

Gechev, T.S., Van Breusegem, F., Stone, J.M., Denev, I. and Laloi, C. (2006) Reactive oxygen species as signals that modulate plant stress responses and programmed cell death. Bioessays 28, 1091-1101.

Godfray, H.C.J., Beddington, J.R., Crute, I.R., Haddad, L., Lawrence, D., Muir, J.F., Pretty, J., Robinson, S., Thomas, S.M. and Toulmin, C. (2010) Food security: the challenge of feeding 9 billion people. Science 327, 812-818.

Gorantla, M., Babu, P., Lachagari, V.B.R., Reddy, A., Wusirika, R., Bennetzen, J.L. and Reddy, A.R. (2007) Identification of stress-responsive genes in an indica rice (Oryza sativa L.) using ESTs generated from drought-stressed seedlings. Journal of experimental botany 58, 253-265.

Guo, T.R., Zhang, G.P. and Zhang, Y.H. (2007) Physiological changes in barley plants under combined toxicity of aluminum, copper and cadmium. Colloids and surfaces B: Biointerfaces 57, 182-188.

Gurley, W.B. (2000) HSP101: a key component for the acquisition of thermotolerance in plants. Science Signaling 12, 457.

Habier, D., Fernando, R.L. and Dekkers, J.C.M. (2009) Genomic selection using low-density marker panels. Genetics 182, 343-353.

Hall, A. (2001) Crop developmental responses to temperature, photoperiod, and light quality. Crop Response to Environment. Boca Raton, FL: CRC, 83-87.

Hall, A.E. (2011) Breeding Cowpea for Future Climates. Crop Adaptation to Climate Change.

Hare, P., Cress, W. and Van Staden, J. (1997) The involvement of cytokinins in plant responses to environmental stress. Plant growth regulation 23, 79-103.

Hedhly, A. (2011) Sensitivity of flowering plant gametophytes to temperature fluctuations. Environmental and Experimental Botany 74, 9-16.

Hedhly, A., Hormaza, J.I. and Herrero, M. (2009) Global warming and sexual plant reproduction. Trends in plant science 14, 30-36.

Heffner, E.L., Sorrells, M.E. and Jannink, J.L. (2009) Genomic selection for crop improvement. Crop Sci 49, 1-12.

Hirayama, T. and Shinozaki, K. (2010) Research on plant abiotic stress responses in the post-genome era: past, present and future. The Plant Journal 61, 1041-1052.

Hong, S.W., Lee, U. and Vierling, E. (2003) Arabidopsis hot mutants define multiple functions required for acclimation to high temperatures. Plant physiology 132, 757-767. 
Hsu, S.F., Lai, H.C. and Jinn, T.L. (2010) Cytosol-localized heat shock factor-binding protein, AtHSBP, functions as a negative regulator of heat shock response by translocation to the nucleus and is required for seed development in Arabidopsis. Plant physiology 153, 773-784.

Hu, X., Li, Y., Li, C., Yang, H., Wang, W. and Lu, M. (2010) Characterization of small heat shock proteins associated with maize tolerance to combined drought and heat stress. Journal of Plant Growth Regulation 29, 455-464.

Huang, B. and Xu, C. (2008) Identification and characterization of proteins associated with plant tolerance to heat stress. Journal of integrative plant biology 50, 1230-1237.

Iba, K. (2002) Acclimative response to temperature stress in higher plants: approaches of gene engineering for temperature tolerance. Annual review of plant biology 53, 225-245.

Ismail, A.M. and Hall, A.E. (1999) Reproductive-stage heat tolerance, leaf membrane thermostability and plant morphology in cowpea. Crop Sci 39, 1762-1768.

Jagadish, S., Muthurajan, R., Oane, R., Wheeler, T.R., Heuer, S., Bennett, J. and Craufurd, P.Q. (2010a) Physiological and proteomic approaches to address heat tolerance during anthesis in rice (Oryza sativa L.). Journal of experimental botany 61, 143-156.

Jagadish, S.V., Muthurajan, R., Oane, R., Wheeler, T.R., Heuer, S., Bennett, J. and Craufurd, P.Q. (2010b) Physiological and proteomic approaches to address heat tolerance during anthesis in rice (Oryza sativa L.). Journal of experimental botany 61, 143-156.

Jaggard, K.W., Qi, A. and Ober, E.S. (2010) Possible changes to arable crop yields by 2050. Philosophical transactions of the Royal Society of London. Series B, Biological sciences $\mathbf{3 6 5}$, 2835-2851.

Jain, M., Prasad, P.V.V., Boote, K.J., Hartwell, A.L. and Chourey, P.S. (2007) Effects of season-long high temperature growth conditions on sugar-to-starch metabolism in developing microspores of grain sorghum (Sorghum bicolor L. Moench). Planta 227, 67-79.

Kagale, S., Divi, U.K., Krochko, J.E., Keller, W.A. and Krishna, P. (2007) Brassinosteroid confers tolerance in Arabidopsis thaliana and Brassica napus to a range of abiotic stresses. Planta 225, 353-364.

Kaya, H., Shibahara, K., Taoka, K., Iwabuchi, M., Stillman, B. and Araki, T. (2001) FASCIATA Genes for Chromatin Assembly Factor-1 in Arabidopsis Maintain the Cellular Organization of Apical Meristems. Cell 104, 131-142.

Khraiwesh, B., Zhu, J.K. and Zhu, J. (2012) Role of miRNAs and siRNAs in biotic and abiotic stress responses of plants. Biochimica et Biophysica Acta (BBA)-Gene Regulatory Mechanisms 1819, 137-148.

Kim, M., Lee, U., Small, I., des Francs-Small, C.C. and Vierling, E. (2012) Mutations in an Arabidopsis Mitochondrial Transcription Termination Factor-Related Protein Enhance Thermotolerance in the Absence of the Major Molecular Chaperone HSP101. The Plant cell 24, 3349-3365.

Königshofer, H., Tromballa, H.W. and Löppert, H.G. (2008) Early events in signalling hightemperature stress in tobacco BY2 cells involve alterations in membrane fluidity and enhanced hydrogen peroxide production. Plant, cell \& environment 31, 1771-1780.

Kotak, S., Larkindale, J., Lee, U., von Koskull-Döring, P., Vierling, E. and Scharf, K.D. (2007) Complexity of the heat stress response in plants. Current opinion in plant biology 10, 310 316.

Kumar, S.V. and Wigge, P.A. (2010) H2A. Z-Containing Nucleosomes Mediate the Thermosensory Response in $<\mathrm{i}>$ Arabidopsis $</ \mathrm{i}>$. Cell 140, 136-147.

Lang-Mladek, C., Popova, O., Kiok, K., Berlinger, M., Rakic, B., Aufsatz, W., Jonak, C., Hauser, M.T. and Luschnig, C. (2010) Transgenerational inheritance and resetting of stress-induced loss of epigenetic gene silencing in Arabidopsis. Molecular plant 3, 594-602.

Larkindale, J., Hall, J.D., Knight, M.R. and Vierling, E. (2005) Heat stress phenotypes of Arabidopsis mutants implicate multiple signaling pathways in the acquisition of thermotolerance. Plant physiology 138, 882-897.

Larkindale, J. and Huang, B. (2004) Thermotolerance and antioxidant systems in Agrostis stolonifera: Involvement of salicylic acid, abscisic acid, calcium, hydrogen peroxide, and ethylene. Journal of plant physiology 161, 405-413. 
Larkindale, J. and Vierling, E. (2008) Core genome responses involved in acclimation to high temperature. Plant physiology 146, 748-761.

Leivar, P. and Quail, P.H. (2011) PIFs: pivotal components in a cellular signaling hub. Trends in plant science 16, 19-28.

Li, M., Ji, L., Yang, X., Meng, Q. and Guo, S. (2012) The protective mechanisms of CaHSP26 in transgenic tobacco to alleviate photoinhibition of PSII during chilling stress. Plant cell reports 31, 1969-1979.

Liu, H. and Charng, Y. (2012) Acquired thermotolerance independent of heat shock factor A1 (HsfA1), the master regulator of the heat stress response. Plant signaling \& behavior 7, 0--1.

Liu, X. and Huang, B. (2000) Carbohydrate accumulation in relation to heat stress tolerance in two creeping bentgrass cultivars. Journal of the American Society for Horticultural Science 125, 442-447.

Lohar, D. and Peat, W. (1998) Floral characteristics of heat-tolerant and heat-sensitive tomato $(<\mathrm{i}>$ Lycopersicon esculentum</i $>$ Mill.) cultivars at high temperature. Scientia Horticulturae 73, 53-60.

Lopes, M.S. and Reynolds, M.P. (2010) Partitioning of assimilates to deeper roots is associated with cooler canopies and increased yield under drought in wheat. Functional plant biology 37, 147-156.

Lotze-Campen, H. (2011) Regional Climate Impacts on Agriculture in Europe. Crop Adaptation to Climate Change.

Mach, J. (2012) Calcium Channels and Acquired Thermotolerance: Here Comes the Sun and It's All Right. The Plant Cell Online 24, 3167-3167.

Maestri, E., Klueva, N., Perrotta, C., Gulli, M., Nguyen, H.T. and Marmiroli, N. (2002) Molecular genetics of heat tolerance and heat shock proteins in cereals. Plant molecular biology 48, 667681.

Mahmood, S., Wahid, A., Javed, F. and Basra, S.M.A. (2010) Heat stress effects on forage quality characteristics of maize (Zea mays) cultivars. International Journal of Agriculture and Biology 12, 701-706.

Mason, R.E., Mondal, S., Beecher, F.W., Pacheco, A., Jampala, B., Ibrahim, A.M.H. and Hays, D.B. (2010) QTL associated with heat susceptibility index in wheat (Triticum aestivum L.) under short-term reproductive stage heat stress. Euphytica 174, 423-436.

Meiri, D., Tazat, K., Cohen-Peer, R., Farchi-Pisanty, O., Aviezer-Hagai, K., Avni, A. and Breiman, A. (2010) Involvement of Arabidopsis ROF2 (FKBP65) in thermotolerance. Plant molecular biology 72, 191-203.

Miller, G. and Mittler, R. (2006) Could heat shock transcription factors function as hydrogen peroxide sensors in plants? Annals of botany 98, 279-288.

Mirzaei, M., Pascovici, D., Atwell, B.J. and Haynes, P.A. (2012) Differential regulation of aquaporins, small GTPases and V-ATPases proteins in rice leaves subjected to drought stress and recovery. Proteomics 12, 864-877.

Mittler, R., Vanderauwera, S., Gollery, M. and Van Breusegem, F. (2004) Reactive oxygen gene network of plants. Trends in plant science 9, 490-498.

Mittler, R., Vanderauwera, S., Suzuki, N., Miller, G., Tognetti, V.B., Vandepoele, K., Gollery, M., Shulaev, V. and Van Breusegem, F. (2011) ROS signaling: the new wave? Trends in plant science 16, 300-309.

Mlynárová, L., Nap, J.P. and Bisseling, T. (2007) The SWI/SNF chromatin-remodeling gene AtCHR12 mediates temporary growth arrest in Arabidopsis thaliana upon perceiving environmental stress. The Plant Journal 51, 874-885.

Morales, D., Rodríguez, P., Dell'Amico, J., Nicolas, E., Torrecillas, A. and Sánchez-Blanco, M.J. (2003) High-temperature preconditioning and thermal shock imposition affects water relations, gas exchange and root hydraulic conductivity in tomato. Biologia plantarum 47, 203-208.

Morgante, M. and Salamini, F. (2003) From plant genomics to breeding practice. Current opinion in biotechnology 14, 214-219. 
Musatenko, L., Vedenicheva, N., Vasyuk, V., Generalova, V., Martyn, G. and Sytnik, K. (2003) Phytohormones in seedlings of maize hybrids differing in their tolerance to high temperatures. Russian journal of plant physiology 50, 444-448.

Nagarajan, S., Jagadish, S., Prasad, A., Thomar, A., Anand, A., Pal, M. and Agarwal, P. (2010) Local climate affects growth, yield and grain quality of aromatic and non-aromatic rice in northwestern India. Agriculture, ecosystems \& environment 138, 274-281.

Nakashima, K., Tran, L.S.P., Van Nguyen, D., Fujita, M., Maruyama, K., Todaka, D., Ito, Y., Hayashi, N., Shinozaki, K. and Yamaguchi-Shinozaki, K. (2007) Functional analysis of a NAC-type transcription factor OsNAC6 involved in abiotic and biotic stress-responsive gene expression in rice. The Plant Journal 51, 617-630.

Nava, G.A., Dalmago, G.A., Bergamaschi, H., Paniz, R., dos Santos, R.P. and Marodin, G.A.B. (2009) Effect of high temperatures in the pre-blooming and blooming periods on ovule formation, pollen grains and yield of 'Granada'peach. Scientia Horticulturae 122, 37-44.

Nelson, G.C. (2009) Climate change: Impact on agriculture and costs of adaptation:International Food Policy Research Inst.

Nover, L., Bharti, K., Döring, P., Mishra, S.K., Ganguli, A. and Scharf, K.D. (2001) Arabidopsis and the heat stress transcription factor world: how many heat stress transcription factors do we need? Cell stress \& chaperones $\mathbf{6}, 177$.

Ogweno, J.O., Song, X.S., Shi, K., Hu, W.H., Mao, W.H., Zhou, Y.H., Yu, J.Q. and Nogués, S. (2008) Brassinosteroids alleviate heat-induced inhibition of photosynthesis by increasing carboxylation efficiency and enhancing antioxidant systems in Lycopersicon esculentum. Journal of Plant Growth Regulation 27, 49-57.

Ortiz, R., Braun, H.J., Crossa, J., Crouch, J.H., Davenport, G., Dixon, J., Dreisigacker, S., Duveiller, E., He, Z.H., Huerta, J., Joshi, A.K., Kishii, M., Kosina, P., Manes, Y., Mezzalama, M., Morgounov, A., Murakami, J., Nicol, J., Ferrara, G.O., Ortiz-Monasterio, J.I., Payne, T.S., Pena, R.J., Reynolds, M.P., Sayre, K.D., Sharma, R.C., Singh, R.P., Wang, J.K., Warburton, M., Wu, H.X. and Iwanaga, M. (2008) Wheat genetic resources enhancement by the International Maize and Wheat Improvement Center (CIMMYT). Genet Resour Crop Ev 55, 1095-1140.

Oshino, T., Abiko, M., Saito, R., Ichiishi, E., Endo, M., Kawagishi-Kobayashi, M. and Higashitani, A. (2007) Premature progression of anther early developmental programs accompanied by comprehensive alterations in transcription during high-temperature injury in barley plants. Molecular Genetics and Genomics 278, 31-42.

Pachauri, R.K. (2007) Sustainable well-being. Science 315, 913.

Paliwal, R., Röder, M.S., Kumar, U., Srivastava, J. and Joshi, A.K. (2012) QTL mapping of terminal heat tolerance in hexaploid wheat (T. aestivum L.). TAG Theoretical and Applied Genetics, 115.

Panchuk, II, Volkov, R.A. and Schoffl, F. (2002) Heat stress- and heat shock transcription factordependent expression and activity of ascorbate peroxidase in Arabidopsis. Plant physiology 129, 838-853.

Parish, R.W., Phan, H.A., Iacuone, S. and Li, S.F. (2012) Tapetal development and abiotic stress: a centre of vulnerability. Functional plant biology 39, 553-559.

Pecinka, A., Dinh, H.Q., Baubec, T., Rosa, M., Lettner, N. and Scheid, O.M. (2010) Epigenetic regulation of repetitive elements is attenuated by prolonged heat stress in Arabidopsis. The Plant Cell Online 22, 3118-3129.

Peet, M., Sato, S. and Gardner, R. (2002) Comparing heat stress effects on male-fertile and malesterile tomatoes. Plant, cell \& environment 21, 225-231.

Pinto, R.S., Reynolds, M.P., Mathews, K.L., McIntyre, C.L., Olivares-Villegas, J.J. and Chapman, S.C. (2010) Heat and drought adaptive QTL in a wheat population designed to minimize confounding agronomic effects. TAG Theoretical and Applied Genetics 121, 1001-1021.

Porter, J.R. and Semenov, M.A. (2005) Crop responses to climatic variation. Philosophical Transactions of the Royal Society B: Biological Sciences 360, 2021-2035. 
Potters, G., Pasternak, T.P., Guisez, Y. and JANSEN, M.A.K. (2008) Different stresses, similar morphogenic responses: integrating a plethora of pathways. Plant, cell \& environment $\mathbf{3 2}$, 158-169.

Potters, G., Pasternak, T.P., Guisez, Y., Palme, K.J. and Jansen, M.A.K. (2007) Stress-induced morphogenic responses: growing out of trouble? Trends in plant science 12, 98-105.

Pradhan, G., Prasad, P., Fritz, A., Kirkham, M. and Gill, B. (2012a) High Temperature Tolerance in Species and Its Potential Transfer to Wheat. Crop Sci 52, 292-304.

Pradhan, G.P., Prasad, P.V.V., Fritz, A.K., Kirkham, M.B. and Gill, B.S. (2012b) Response of Aegilops species to drought stress during reproductive stages of development. Functional plant biology 39, 51-59.

Prasad, N.G., Dey, S., Shakarad, M. and Joshi, A. (2003) The evolution of population stability as a by-product of life-history evolution. Proceedings. Biological sciences / The Royal Society $\mathbf{2 7 0}$ Suppl 1, S84-86.

Prasad, P., Boote, K., Allen, L., Sheehy, J. and Thomas, J. (2006) Species, ecotype and cultivar differences in spikelet fertility and harvest index of rice in response to high temperature stress. Field Crops Research 95, 398-411.

Prasad, P., Pisipati, S., Mutava, R. and Tuinstra, M. (2008) Sensitivity of grain sorghum to high temperature stress during reproductive development. Crop Sci 48, 1911-1917.

Pressman, E., Peet, M.M. and Pharr, D.M. (2002) The effect of heat stress on tomato pollen characteristics is associated with changes in carbohydrate concentration in the developing anthers. Annals of botany 90, 631-636.

Qi, Y., Wang, H., Zou, Y., Liu, C., Liu, Y., Wang, Y. and Zhang, W. (2011) Over-expression of mitochondrial heat shock protein 70 suppresses programmed cell death in rice. FEBS letters $\mathbf{5 8 5}, 231-239$.

Quan, R., Shang, M., Zhang, H., Zhao, Y. and Zhang, J. (2004) Engineering of enhanced glycine betaine synthesis improves drought tolerance in maize. Plant biotechnology journal 2, 477 486.

Queitsch, C., Hong, S.W., Vierling, E. and Lindquist, S. (2000) Heat shock protein 101 plays a crucial role in thermotolerance in Arabidopsis. The Plant cell 12, 479-492.

Ramalho, M.A.P., Abreu, Â.F.B. and Santos, J.B. (2005) Genetic progress after four cycles of recurrent selection for yield and grain traits in common bean. Euphytica 144, 23-29.

Rasheed, R. (2009) Salinity and extreme temperature effects on sprouting buds of sugarcane Saccharum officinarum L.): Some histological and biochemical studies. Ph. D. thesis, Department of Botany, University of Agriculture, Faisalabad, Pakistan.

Ren, C., Bilyeu, K.D. and Beuselinck, P. (2009) Composition, vigor, and proteome of mature soybean seeds developed under high temperature. Crop Sci 49, 1010-1022.

Ristic, Z., Momčilović, I., Bukovnik, U., Prasad, P., Fu, J., DeRidder, B.P., Elthon, T.E. and Mladenov, N. (2009) Rubisco activase and wheat productivity under heat-stress conditions. Journal of experimental botany 60, 4003-4014.

Rivero, R.M., Ruiz, J.M., García, P.C., López-Lefebre, L.R., Sánchez, E. and Romero, L. (2001) Resistance to cold and heat stress: accumulation of phenolic compounds in tomato and watermelon plants. Plant Science 160, 315-321.

Roitsch, T. and González, M.C. (2004) Function and regulation of plant invertases: sweet sensations. Trends in plant science $9,606-613$.

Roy, S.J., Tucker, E.J. and Tester, M. (2011) Genetic analysis of abiotic stress tolerance in crops. Current opinion in plant biology 14, 232-239.

Ruan, Y.-L., Jin, Y., Yang, Y.-J., Li, G.-J. and Boyer, J.S. (2010) Sugar input, metabolism, and signaling mediated by invertase: roles in development, yield potential, and response to drought and heat. Molecular plant 3, 942-955.

Saha, S., Hossain, M., Rahman, M., Kuo, C. and Abdullah, S. (2010) Effect of high temperature stress on the performance of twelve sweet pepper genotypes. Bangladesh Journal of Agricultural Research 35, 525-534.

Saidi, Y., Finka, A. and Goloubinoff, P. (2011) Heat perception and signalling in plants: a tortuous path to thermotolerance. New Phytologist 190, 556-565. 
Sairam, R. and Tyagi, A. (2004) Physiology and molecular biology of salinity stress tolerance in plants. CURRENT SCIENCE-BANGALORE- 86, 407-421.

Sakamoto, A. and Murata, N. (2000) Genetic engineering of glycinebetaine synthesis in plants: current status and implications for enhancement of stress tolerance. Journal of experimental botany 51, 81-88.

Sakamoto, A. and Murata, N. (2002) The role of glycine betaine in the protection of plants from stress: clues from transgenic plants. Plant, cell \& environment 25, 163-171.

Sakata, T. and Higashitani, A. (2008) Male sterility accompanied with abnormal anther development in plants-genes and environmental stresses with special reference to high temperature injury. International Journal of Plant Developmental Biology 2, 42-51.

Sakata, T., Oshino, T., Miura, S., Tomabechi, M., Tsunaga, Y., Higashitani, N., Miyazawa, Y., Takahashi, H., Watanabe, M. and Higashitani, A. (2010) Auxins reverse plant male sterility caused by high temperatures. Proceedings of the National Academy of Sciences 107, 85698574.

Salvucci, M.E. and Crafts-Brandner, S.J. (2004) Inhibition of photosynthesis by heat stress: the activation state of Rubisco as a limiting factor in photosynthesis. Physiologia plantarum 120, 179-186.

Sánchez-Rodríguez, E., Moreno, D.A., Ferreres, F., Rubio-Wilhelmi, M.M. and Ruiz, J.M. (2011) Differential responses of five cherry tomato varieties to water stress: changes on phenolic metabolites and related enzymes. Phytochemistry 72, 723-729.

Sato, S., Kamiyama, M., Iwata, T., Makita, N., Furukawa, H. and Ikeda, H. (2006) Moderate increase of mean daily temperature adversely affects fruit set of Lycopersicon esculentum by disrupting specific physiological processes in male reproductive development. Annals of botany 97, 731-738.

Savchenko, G., Klyuchareva, E., Abramchik, L. and Serdyuchenko, E. (2002) Effect of periodic heat shock on the inner membrane system of etioplasts. Russian journal of plant physiology $\mathbf{4 9}$, 349-359.

Savin, R. and Slafer, G.A. (2010) Agricultural production and yield. Agricultural Sciences: Topics in Modern Agriculture, 291.

Scafaro, A.P., Haynes, P.A. and Atwell, B.J. (2010) Physiological and molecular changes in Oryza meridionalis $\mathrm{Ng}$., a heat-tolerant species of wild rice. Journal of experimental botany 61, 191202.

Scharf, K.D., Berberich, T., Ebersberger, I. and Nover, L. (2011) The plant heat stress transcription factor (Hsf) family: structure, function and evolution. Biochimica et Biophysica Acta (BBA)Gene Regulatory Mechanisms.

Schramm, F., Larkindale, J., Kiehlmann, E., Ganguli, A., Englich, G., Vierling, E. and Von KoskullDöring, P. (2008) A cascade of transcription factor DREB2A and heat stress transcription factor HsfA3 regulates the heat stress response of Arabidopsis. The Plant Journal 53, 264274.

Selvaraj, M.G., Burow, G., Burke, J.J., Belamkar, V., Puppala, N. and Burow, M.D. (2011) Heat stress screening of peanut (Arachis hypogaea L.) seedlings for acquired thermotolerance. Plant growth regulation 65, 83-91.

Semenov, M.A. and Halford, N.G. (2009) Identifying target traits and molecular mechanisms for wheat breeding under a changing climate. Journal of experimental botany 60, 2791-2804.

Shah, N. and Paulsen, G. (2003) Interaction of drought and high temperature on photosynthesis and grain-filling of wheat. Plant and Soil 257, 219-226.

Sharkey, T.D. and Zhang, R. (2010) High temperature effects on electron and proton circuits of photosynthesis. Journal of integrative plant biology 52, 712-722.

Singh, R.K., Redoña, E. and Refuerzo, L. (2010) Varietal improvement for abiotic stress tolerance in crop plants: special reference to salinity in rice. Abiotic Stress Adaptation in Plants, 387-415.

Sivasankar, S., Williams, R.W. and Greene, T.W. (2012) Abiotic Stress Tolerance in Plants: An Industry Perspective. Improving Crop Resistance to Abiotic Stress, 27-47.

Smertenko, A., DRÁBER, P., Viklický, V. and Opatrný, Z. (1997) Heat stress affects the organization of microtubules and cell division in Nicotiana tabacum cells. Plant, cell \& environment 20 , 1534-1542. 
Smith, K.T. and Workman, J.L. (2012) Chromatin Proteins: Key Responders to Stress. PLoS Biology 10, e1001371.

Souza, M.A., Pimentel, A.J.B. and Ribeiro, G. (2012) Breeding for Heat-Stress Tolerance. Plant Breeding for Abiotic Stress Tolerance, 137-156.

Sugio, A., Dreos, R., Aparicio, F. and Maule, A.J. (2009) The cytosolic protein response as a subcomponent of the wider heat shock response in Arabidopsis. The Plant Cell Online 21, 642-654.

Sumesh, K., Sharma-Natu, P. and Ghildiyal, M. (2008) Starch synthase activity and heat shock protein in relation to thermal tolerance of developing wheat grains. Biologia plantarum $\mathbf{5 2}$, 749-753.

Sun, W., Van Montagu, M. and Verbruggen, N. (2002) Small heat shock proteins and stress tolerance in plants. Biochimica et biophysica acta 1577, 1-9.

Sung, D.Y., Kaplan, F., Lee, K.J. and Guy, C.L. (2003) Acquired tolerance to temperature extremes. Trends in plant science $\mathbf{8}, 179-187$.

Suzuki, K., Takeda, H., Tsukaguchi, T. and Egawa, Y. (2001) Ultrastructural study on degeneration of tapetum in anther of snap bean (Phaseolus vulgaris L.) under heat stress. Sexual Plant Reproduction 13, 293-299.

Suzuki, N., Miller, G., Sejima, H., Harper, J. and Mittler, R. (2013) Enhanced seed production under prolonged heat stress conditions in Arabidopsis thaliana plants deficient in cytosolic ascorbate peroxidase 2. Journal of experimental botany 64, 253-263.

Taiz, L. and Zeiger, E. (2006) Stress physiology. Plant Physiology, Taiz, L. and E. Zeiger (Eds.). Sinauer Associates, Inc., Sunderland, MA, 671-681.

Takeda, S. and Matsuoka, M. (2008) Genetic approaches to crop improvement: responding to environmental and population changes. 9, 444-457.

Talanova, V., Akimova, T. and Titov, A. (2003) Effect of whole plant and local heating on the ABA content in cucumber seedling leaves and roots and on their heat tolerance. Russian journal of plant physiology 50, 90-94.

Tauber, E., Zordan, M., Sandrelli, F., Pegoraro, M., Osterwalder, N., Breda, C., Daga, A., Selmin, A., Monger, K., Benna, C., Rosato, E., Kyriacou, C.P. and Costa, R. (2007) Natural selection favors a newly derived timeless allele in Drosophila melanogaster. Science 316, 1895-1898.

Tester, M. and Langridge, P. (2010) Breeding technologies to increase crop production in a changing world. Science 327, 818-822.

Thakur, P., Kumar, S., Malik, J.A., Berger, J.D. and Nayyar, H. (2010) Cold stress effects on reproductive development in grain crops: an overview. Environmental and Experimental Botany 67, 429-443.

Thomson, M.J., de Ocampo, M., Egdane, J., Rahman, M.A., Sajise, A.G., Adorada, D.L., Tumimbang-Raiz, E., Blumwald, E., Seraj, Z.I. and Singh, R.K. (2010) Characterizing the Saltol quantitative trait locus for salinity tolerance in rice. Rice 3, 148-160.

Todorov, D., Karanov, E., Smith, A.R. and Hall, M.A. (2003) Chlorophyllase activity and chlorophyll content in wild and mutant plants of Arabidopsis thaliana. Biologia plantarum 46, 125-127.

Varshney, R.K., Bansal, K.C., Aggarwal, P.K., Datta, S.K. and Craufurd, P.Q. (2011) Agricultural biotechnology for crop improvement in a variable climate: hope or hype? Trends in plant science 16, 363-371.

Varshney, R.K., Nayak, S.N., May, G.D. and Jackson, S.A. (2009) Next-generation sequencing technologies and their implications for crop genetics and breeding. Trends in biotechnology 27, 522-530.

Velikova, V., Pinelli, P., Pasqualini, S., Reale, L., Ferranti, F. and Loreto, F. (2005) Isoprene decreases the concentration of nitric oxide in leaves exposed to elevated ozone. New Phytologist 166, 419-426.

Vierling, E. (1991) The roles of heat shock proteins in plants. Annual review of plant biology 42, 579620.

Vijayalakshmi, K., Fritz, A.K., Paulsen, G.M., Bai, G., Pandravada, S. and Gill, B.S. (2010) Modeling and mapping QTL for senescence-related traits in winter wheat under high temperature. Molecular Breeding 26, 163-175. 
Volkov, R.A., Panchuk, I.I., Mullineaux, P.M. and Schöffl, F. (2006) Heat stress-induced H 2 O 2 is required for effective expression of heat shock genes in Arabidopsis. Plant molecular biology 61, 733-746.

Vollenweider, P. and Günthardt-Goerg, M.S. (2005) Diagnosis of abiotic and biotic stress factors using the visible symptoms in foliage. Environmental pollution 137, 455-465.

Vos, P., Hogers, R., Bleeker, M., Reijans, M., van de Lee, T., Hornes, M., Frijters, A., Pot, J., Peleman, J., Kuiper, M. and et al. (1995) AFLP: a new technique for DNA fingerprinting. Nucleic acids research $\mathbf{2 3}, 4407-4414$.

Wahid, A. (2007) Physiological implications of metabolite biosynthesis for net assimilation and heatstress tolerance of sugarcane (Saccharum officinarum) sprouts. Journal of plant research 120, 219-228.

Wahid, A. and Close, T. (2007) Expression of dehydrins under heat stress and their relationship with water relations of sugarcane leaves. Biologia plantarum 51, 104-109.

Wahid, A., Gelani, S., Ashraf, M. and Foolad, M. (2007) Heat tolerance in plants: an overview. Environmental and Experimental Botany 61, 199-223.

Wahid, A. and Shabbir, A. (2005) Induction of heat stress tolerance in barley seedlings by pre-sowing seed treatment with glycinebetaine. Plant growth regulation 46, 133-141.

Wang, X., Liu, L., Liu, S., Sun, X., Deng, Z., Pi, Y. and Tang, K. (2004) Isolation and molecular characterization of a new CRT binding factor gene from Capsella bursa-pastoris. Journal of biochemistry and molecular biology 37, 538-545.

Wassmann, R., Jagadish, S., Sumfleth, K., Pathak, H., Howell, G., Ismail, A., Serraj, R., Redona, E., Singh, R. and Heuer, S. (2009) Regional vulnerability of climate change impacts on Asian rice production and scope for adaptation. Advances in Agronomy 102, 91-133.

Willits, D.H. and Peet, M.M. (1998) The effect of night temperature on greenhouse grown tomato yields in warm climates. Agr Forest Meteorol 92, 191-202.

Wise, R., Olson, A., Schrader, S. and Sharkey, T. (2004) Electron transport is the functional limitation of photosynthesis in field-grown pima cotton plants at high temperature. Plant, cell \& environment 27, 717-724.

Xu, S., Li, J., Zhang, X., Wei, H. and Cui, L. (2006) Effects of heat acclimation pretreatment on changes of membrane lipid peroxidation, antioxidant metabolites, and ultrastructure of chloroplasts in two cool-season turfgrass species under heat stress. Environmental and Experimental Botany 56, 274-285.

Yamamoto, K., Sakamoto, H. and Momonoki, Y.S. (2011) Maize acetylcholinesterase is a positive regulator of heat tolerance in plants. Journal of plant physiology 168, 1987-1992.

Yang, J., Sears, R., Gill, B. and Paulsen, G. (2002) Quantitative and molecular characterization of heat tolerance in hexaploid wheat. Euphytica 126, 275-282.

Yang, J. and Zhang, J. (2010) Grain-filling problem in 'super'rice. Journal of experimental botany 61, $1-5$.

Ye, C., Argayoso, M.A., Redoña, E.D., Sierra, S.N., Laza, M.A., Dilla, C.J., Mo, Y., Thomson, M.J., Chin, J. and Delaviña, C.B. (2012) Mapping QTL for heat tolerance at flowering stage in rice using SNP markers. Plant Breeding.

Yoshida, T., Ohama, N., Nakajima, J., Kidokoro, S., Mizoi, J., Nakashima, K., Maruyama, K., Kim, J.M., Seki, M. and Todaka, D. (2011) Arabidopsis HsfA1 transcription factors function as the main positive regulators in heat shock-responsive gene expression. Molecular Genetics and Genomics 286, 321-332.

Young, L.W., Wilen, R.W. and Bonham-Smith, P.C. (2004) High temperature stress of Brassica napus during flowering reduces micro-and megagametophyte fertility, induces fruit abortion, and disrupts seed production. Journal of experimental botany 55, 485-495.

Zhang, J., Huang, W., Pan, Q. and Liu, Y. (2005a) Improvement of chilling tolerance and accumulation of heat shock proteins in grape berries $(<\mathrm{i}>$ Vitis vinifera $</ \mathrm{i}>\mathrm{cv}$. Jingxiu) by heat pretreatment. Postharvest biology and technology 38, 80-90.

Zhang, J.X., Wang, C., Yang, C.Y., Wang, J.Y., Chen, L., Bao, X.M., Zhao, Y.X., Zhang, H. and Liu, J. (2010) The role of Arabidopsis AtFes1A in cytosolic Hsp70 stability and abiotic stress tolerance. The Plant journal : for cell and molecular biology 62, 539-548. 
Zhang, M., Barg, R., Yin, M., Gueta-Dahan, Y., Leikin-Frenkel, A., Salts, Y., Shabtai, S. and BenHayyim, G. (2005b) Modulated fatty acid desaturation via overexpression of two distinct omega-3 desaturases differentially alters tolerance to various abiotic stresses in transgenic tobacco cells and plants. The Plant journal : for cell and molecular biology 44, 361-371.

Zhang, W.B., Jiang, H., Qiu, P.C., Liu, C.Y., Chen, F.L., Xin, D.W., Li, C.D., Hu, G.H. and Chen, Q.S. (2012) Genetic overlap of QTL associated with low-temperature tolerance at germination and seedling stage using BILs in soybean. Canadian Journal of Plant Science $\mathbf{9 2}$, $1-8$.

Zhang, X. and Ervin, E. (2008) Impact of seaweed extract-based cytokinins and zeatin riboside on creeping bentgrass heat tolerance. Crop Sci 48, 364-370.

Zinn, K.E., Tunc-Ozdemir, M. and Harper, J.F. (2010) Temperature stress and plant sexual reproduction: uncovering the weakest links. Journal of experimental botany 61, 1959-1968. 


\section{Chapter 3}

\section{Temporal and spatial effects of moderate heat stress on gene expression in meiotic tomato anthers}




\begin{abstract}
Sexual reproduction and flowering in particular have been long recognized as extremely sensitive to heat stress, which often results in reduced crop plant productivity. Fluctuations in temperature occur naturally during plant growth and reproduction. However, in hot summers this variation can damage the fine intermolecular interactions needed for proper growth, impairing plant development and more importantly for many crop plants, fruit-set. We have used cDNA-AFLP to profile the general response of tomato meiotic anthers to moderate heat stress conditions (MHS) $\left(32^{\circ} \mathrm{C}\right)$. Our results reveal that after $30 \mathrm{~h}$ of MHS approximately $1 \%$ of the examined transcript-derived fragments exhibited alterations in expression pattern. The majority of these were down-regulated after the first $2 \mathrm{~h}$ of stress. The putative functions associated with the genes identified by cDNA-AFLP indicate involvement of heat shock, metabolism, antioxidant and developmental processes. Based upon the observed differences in response to MHS and on literature sources, we identified a number of candidate transcripts involved in the response to heat. Elucidating the mechanisms of heat tolerance might be particularly advantageous in the light of the expected global temperature increases. The expression of several candidate genes was further investigated using in situ hybridisation and we were able to show that the investigated genes are expressed in the tapetum and developing microspores.
\end{abstract}




\section{Introduction}

Temperature stress affects different plant developmental and physiological processes and is considered one of the major constrains to plant adaptation, especially when it coincides with critical stages of plant development (Hall, 2001).

Male reproductive development in higher plants is known to be very sensitive to abiotic stress. In particular, high or low temperature stress results in a lower seed set due to male sterility in most crops, including tomatoes (Hedhly, 2011; Sato et al., 2006). The developmental pathway for a pollen grain starts with the separation of the reproductive tissues of the anther, continues with meiosis of the pollen mother cell, followed by mitosis and microspore maturation that results in the mature pollen grain. After initiation, the highly specialized anther tissues will acquire non-reproductive (e.g., the tapetum for support, stomium for dehiscence) or reproductive functions (egg cell and pollen formation). Both tapetum and microspore development are essential for male fertility, as documented by numerous studies on male sterile mutants (Bartels, 2001; Bolouri-Moghaddam et al., 2010; Levy et al., 1978; Peet, 1998; Peet et al., 1998; Peshev and Van den Ende, 2012; Sato et al., 2004).

The tapetum is important to microspore development as it not only provides callase for the digestion of the callose surrounding the microspore tetrad, but also nutrition and sporopollenin needed to construct the pollen exine after tetrad dissolution (Bedinger, 1992). In Petunia, a cDNA-AFLP study revealed that during meiosis in anthers under standard conditions, besides the typical meiotic genes, other genes are also important for the development of the pollen grains. Thus, during meiosis, tapetum differentiation genes, serine-proteinases, hormone precursors, genes involved in cell wall biosynthesis and even ribonucleases and polyamine biosynthesis genes are all modulated in expression (Shen et al., 1997).

Although tomato plants can grow under a wide range of temperatures, optimal fruit set and fruit weight are limited to a somewhat narrower range. Pollen development is more sensitive to heat, as no fruit develops on male sterile flowers receiving pollen developed at high temperature. The alterations observed in anther/developing pollen grains can drastically reduce the number of released pollen and pollen viability, and, consequently, significantly decrease the ability of tomato plants to set fruit. It seems thus that pollen development and release are among the major limiting factors for fruit set in tomato, under high temperatures (Kaya et al., 2012).

During male reproductive development, the meiotic stage is known to be highly sensitive to environmental stress. At the time of pollen meiosis, the tapetum is very active and the anther is the strongest sink organ in the flower (Feder and Hofmann, 1999). This may explain the higher vulnerability of pollen development to abiotic stresses. High temperatures are known to limit fruit set in tomatoes due to a simultaneously and/or sequentially impaired series of reproductive processes (Larkindale and Knight, 2002). At temperatures typical for warm-temperate regions $\left(32^{\circ} \mathrm{C} / 26^{\circ} \mathrm{C}\right)$ fruit set decreases with temperature increase, due to abnormalities in anther development, especially in tissues with important roles in pollen development and release, such as tapetum and stomium. For example, in rice, wheat, snap bean or pepper anthers grown in high temperature conditions, tapetal degradation fails to occur and the pollen grain wall appears to be affected, consistent with disruption of tapetum function (Chinnusamy et al., 2008; Endo et al., 2009; Gibson, 2003; Suzuki et al., 2001). In tomato, a defective tapetum is responsible for 11 of 23 sporogenous male sterile mutants previously described (Sreenivasulu et al., 2012).

Plant development and the response to environmental stimuli result in rapid changes of gene transcription. In general such changes are mediated through a signalling transduction pathway that starts with either master a regulator or receptors initiating a cascade of gene induction that evokes a developmental progression and/or the responses to the environmental situation. Such responses invariably involve a change in flux though metabolic pathways and the attendant coordinated 
transcriptional regulation of the genes coding for the enzymes in that pathway. It is well known that abiotic stresses in general, through regulation of both gene expression and protein turnover, alter the abundance of many transcripts and proteins (Arenas-Huertero et al., 2000; Mittler, 2006), indicating that transcriptional and post-transcriptional regulation play an essential role in the adaptation of cellular functions to the environmental changes. Understanding how different plants cope with stress during their reproductive (gametophytic) phase is thus critical to managing the future of agricultural productivity (Zinn et al., 2010).

Amplified fragment length polymorphism (AFLP) is a technique for the visualisation of genomic DNA polymorphisms within a genome (Vos et al., 1995). As a further extension of the application, the method was applied to cDNA with the aim of visualising gene expression rather than detecting DNA-polymorphism (cDNA-AFLP) (Bachem et al., 1996). The intensity of individual signals in the fingerprint is then taken as a measure of the strength of expression of the gene corresponding to the band or "transcript derived fragment" (TDF). Plant development, biotic and abiotic stresses have been investigated using cDNA-AFLP in equal measure (Bachem et al., 1996; Bray et al., 2000). One common theme that has emerged from these experiments is that the transcriptional response initially is composed of a core set of multi-stress responsive genes and becomes increasingly stress-specific as time progresses. These genes are classified into three major groups: (1) those that encode products that directly protect plant cells against stresses such as heat stress proteins (HSPs) or chaperones, LEA proteins, osmo-protectants, antifreeze proteins, detoxification enzymes and free-radical scavengers; (2) those that are involved in signalling cascades and in transcriptional control, such as Mitogen-activated protein kinase (MAPK), Calcium-dependent protein kinase (CDPK) phospholipases and transcriptional factors; (3) those that are involved in water and ion uptake and transport such as aquaporins and ion transporters (Ciarmiello et al., 2011).

To survive the numerous environmental stresses they are challenged with, plants have evolved elaborate mechanisms to perceive and rapidly respond to various abiotic and biotic stresses. An essential component of all those stress responses is the capacity to modify the transcriptome. To better understand plant stress responses, transcript profiling experiments have been successfully employed for many different abiotic and biotic stresses (Walley and Dehesh, 2010). For example, to understand the molecular bases of thermotolerance in cowpea nodules, cDNA-AFLP was used to identify differentially expressed transcripts from cowpea nodules subjected to heat shock treatment. The majority of the transcripts characterized showed significant homology with known proteins, such as Phaseolus vulgaris low molecular weight heat shock protein, Medicago sativa putative woundinduced protein, disease resistance protein, xylan endohydrolase isoenzyme, and the pherophorin protein from Arabidopsis thaliana. The transcripts characterized so far have homology to genes related to stress defence, suggesting that they might play a part in the thermotolerance mechanism (Peng et al., 2006). Similarly to understanding the molecular basis of the high level of temperature tolerance in rice, a differential gene expression analysis by cDNA-AFLP was performed in rice leaves and the identified transcripts were functionally classified as belonging to metabolism (including carbohydrate metabolism, protein metabolism, polyamine metabolism, amino acid metabolism, ribonucleotide metabolism, and cellulose synthesis), transport, stress response, and signal transduction groups, with a significant number of transcripts showing differential expression in the tolerant genotype (Hey et al., 2010).

Moreover, as cDNA-AFLP allows the identification of tissue-specific and low abundance sequences with no prior knowledge of gene sequences, this approach was also adopted for the identification of stress induced transcripts in tomato developing microspores. Thus, pollen-specific functions that are missing from the tomato Affymetrix chip were identified, such as vesicle-mediated transport and calcium signalling. In addition, for several genes higher basal expression levels were 
detected in microspores of the tolerant cultivar compared with microspores of the sensitive cultivar, marking these genes as candidates for taking part in microspore thermotolerance (Frank et al., 2009).

In this chapter we present an analysis of the different changes in gene expression in developing meiotic anthers (the first and most sensitive anther developmental stage) in response to MHS in tomato. Assuming the protective mechanisms are initiated shortly after the commencement of the stress period, we decided to study gene expression changes in tomato meiotic anthers during the first 30h of MHS. cDNA-AFLP was applied to obtain a general overview of molecular mechanisms that participate in the response to MHS of tomato anthers. A comparison of transcription profiles obtained from heat-stressed and non-stressed tomato microspores and a functional classification of the annotated transcripts shows that majority of the genes are involved in stress response and transcription regulation, including HSPs and genes associated with oxidative stress response, while a minority comprise signal transduction, metabolism, transport and cell wall modification-related processes. Accordingly, we describe a set of genes that open up the possibility of investigating and modulating heat tolerance in tomato and other commercially important Solanaceae crops.

\section{Materials and methods}

\section{Plant material and heat stress conditions}

The transcriptome analysis has been performed on meiotic anther of a heat sensitive tomato genotype. In our experience the commercial genotype Moneymaker (MM) is heat sensitive under greenhouse conditions (own observations). Seeds were germinated in potting compost in trays and transferred after two weeks to a growth chamber under standard temperature (ST) conditions, with a 16-h light $\left(26^{\circ} \mathrm{C}\right)$ / 8-h dark $\left(18^{\circ} \mathrm{C}\right)$ cycle. Fluorescent and incandescent lighting provided a photosynthetic photon flux density of $450-500 \mu \mathrm{mol} \mathrm{m}-2 \mathrm{sec}-1$. Six-week-old plants were heat stressed by raising the temperature to a regime of MHS $\left(32^{\circ} \mathrm{C} / 26^{\circ} \mathrm{C}\right.$; day/night). The heat treatment was initiated by progressively increasing the temperature from 25 to $32^{\circ}$ over half an hour period, and samples were collected at 0,2 , 6,16 or $30 \mathrm{~h}$ of MHS. In order to describe the dynamics of transcriptional responses of tomato developing meiotic anthers to MHS, the temperature range for the experiment was chosen based on agronomically relevant temperatures shown to have a significant effect on pollen viability (Sato et al., 2006), rather than using classical heat shock conditions of $42^{\circ}-45^{\circ} \mathrm{C}$ (Frank et al., 2009). MHS was applied to whole plants in the growth chamber according to the following scheme (Figure 2), under the same light conditions as stated above.

\section{RNA extraction and experimental design}

We focused our analysis of gene expression on whole meiotic anther cones isolated from flower buds of plants that were exposed to MHS $\left(32{ }^{\circ} \mathrm{C} / 26{ }^{\circ} \mathrm{C}\right.$, day/night) for up to $30 \mathrm{~h}$, and compared this to standard temperatures $\left(26^{\circ} \mathrm{C} / 18^{\circ} \mathrm{C}\right.$ day/night $)$. For the cDNA-AFLP experiment, a pool of six meiotic flower buds from 3 plants was harvested according to (Wilkinson and Davies, 2010). Pilot experiments indicated the third and fourth flower clusters to give the most consistent phenotypic response to MHS (data not shown). From these, anther cones ranging in size from 2-4 mm were isolated, immediately frozen in liquid N2 and pooled for RNA extraction. Anthers and other plant tissues (leaf, ovary, petal, pistil, root, seedling, sepal, stem) used to analyse tissue specificity of the transcripts were ground or homogenized in liquid nitrogen using glass rods and RNA was extracted using a Plant RNA isolation kit (Plant RNeasy Mini Kit, Qiagen Benelux BV, Venlo the Netherlands) according to the manufacturer's recommendations. 


\section{cDNA-AFLP transcript profiling}

For cDNA-AFLP, material was harvested at $0 \mathrm{~h}(\mathrm{ST})$ and at $2 \mathrm{~h}, 6 \mathrm{~h}, 16 \mathrm{~h}$ and $30 \mathrm{~h}$ from both $\mathrm{ST}$ and MHS treatments. The isolated RNA was subjected to cDNA-AFLP template preparation (Kim et al., 2001). RNA fingerprinting was carried out using 92 primer combinations with 2 selective nucleotides and gave rise to an average of 80 transcript-derived fragments per primer combination (TDFs). Primer sequences were as described in Bachem et al. (Kim et al., 2001). Changes in the intensity of individual bands did not affect others in the same lane, indicating that product accumulation was not affected by the concentration of individual substrates in the reaction. Inconsistent bands were mainly observed in the region of the gel with very small DNA fragments $(<70 \mathrm{bp})$ and were therefore considered as aspecific amplification products. Bands corresponding to differentially expressed genes were cut out from the gel and the eluted DNA was re-amplified under the same conditions as for the selective amplification. Fragments were subsequently ligated in a T-tailed EcoRV digested phagemid (pBlueScriptII SK(+), Stratagene, La Jolla, CA, USA) and sequenced (CEQ ${ }^{\text {TM }}$ DTCS Quick Start Kit and CEQ2000 DNA Analysis System, Beckman Coulter, Fullerton, CA, USA). Fragments that did not have the expected size, based on the height on the acryl amide gel from which they were isolated, were discarded.

\section{Validation of gene expression by real-time PCR (RT-PCR)}

Total DNA-free RNA was isolated from tomato anthers ( $>$ > 5) using a RNA isolation kit (Qiagen RNeasy mini kit with the RNase-Free DNase Set, Qiagen). A PCR reaction with RNA- and DNAspecific primers (Supplementary Materials) based on an intron in the S. lycopersicum actin gene Tom51 (GenBank accession number U60481) was performed to ensure the absence of contaminating genomic DNA. Total DNA-free RNA $(1 \mu \mathrm{g})$ was used for cDNA synthesis (iScriptTM cDNA Synthesis Kit; Bio-Rad Laboratories, Hercules, CA, USA) in a total volume of $25 \mu 1$. PCR reactions were carried out in $25 \mu 1$ containing $0.125 \mu 1$ of cDNA synthesis reaction mixture, $400 \mathrm{~nm}$ of each primer and $12.5 \mu \mathrm{l}$ of iQ SYBR Green Supermix (Bio-Rad Laboratories). PCRs were performed in a 96-well Bio-Rad iCycler (Bio-Rad Laboratories) using a temperature program starting with 3 min at $95^{\circ} \mathrm{C}$ followed by 40 cycles consisting of $15 \mathrm{~s}$ at $95^{\circ} \mathrm{C}$ and $45 \mathrm{~s}$ at $57^{\circ} \mathrm{C}$, and finally the melting temperature of the amplified product was determined to verify the presence of a specific product. In addition, a fraction of the PCR mixture was analysed on a 1\% agarose/ethidium bromide gel to check the size of the amplified DNA fragment. The primers that were used for the real-time quantitative PCR reactions were designed using a computer program (Beacon Designer 5.01; Premier Biosoft International, Palo Alto, CA, USA) to obtain primers that have close to identical melting temperatures and do not form secondary structures with each other in the given PCR conditions. Primer sequences are listed in the Appendix. In addition, to enhance primer efficiency, primer-binding sites were chosen such that secondary structures of the template were avoided. All reactions were performed on a pool of RNA samples, collected independently and pooled after RNA extraction.

\section{In situ RNA hybridizations}

For the in situ hybridization experiments, each of the four 250-bp fragments was amplified and inserted into a pGEM-T Easy vector. The sequences of the primers used for the in situ reactions can be found in the Appendix. The sense and antisense DIG-labelled probes were generated by in vitro transcription using T7 and SP6 RNA polymerases according to manufacturer's protocol (Roche Diagnostics, Indianapolis, IN, US). For this experiment, meiotic anthers were collected from plants treated for $2 \mathrm{~h}$ with MHS $\left(32^{\circ} \mathrm{C}\right)$. Anther fixation, paraplast embedding and in situ hybridisations were performed as described by (Chinnusamy et al., 2004). In addition, tissue specificity analyses were performed by RT-PCR using the same set of primers mentioned above. 


\section{Results and discussion}

\section{Pollen sensitivity under MHS}

Although no detectable changes in pollen viability have been observed after $30 \mathrm{~h}$ of MHS in the Moneymaker genotype (MM), a clear indication of the sensitivity of the genotype is the viability of the pollen developing under MHS. Thus in MM, 1 week of moderate heat stress significantly alters pollen viability, as detected with Alexander staining and by cytological studies. Under control conditions MM shows development of tetrads (Figure 1a, tr) at the $3 \mathrm{~mm}$ stage, while under MHS there is an increase in vacuolisation and degradation of nuclei. These cells appear not to have reached the tetrad stage but rather are likely to represent degraded pollen mother cells (Figure 1b). Under MHS, there are also visible aberrations in the tapetum.

In mature anthers $(7 \mathrm{~mm})$ fully developed pollen is observed, while in the corresponding MHS treated anthers, in the most extreme cases, locules are completely devoid of pollen (Figure 1c, d respectively). In order to assess viability, Alexander staining was also used on $3 \mathrm{~mm}$ anthers. Under control conditions viable pollen can be detected (orange staining, Figure 1e), while the MHS treated anthers show necrotic tissues in the locule indicating cell death and lack of viable pollen (dark grey staining, Figure 1f). Collectively, these macroscopic and histological observations provide evidence that the temperature regime was severe enough to trigger visible symptoms of injury that would negatively impact male gametophyte development. However, the imposed temperature treatments were not lethal, as after 2 weeks of additional growth in control conditions the plants were able to produce viable fruits (not shown).

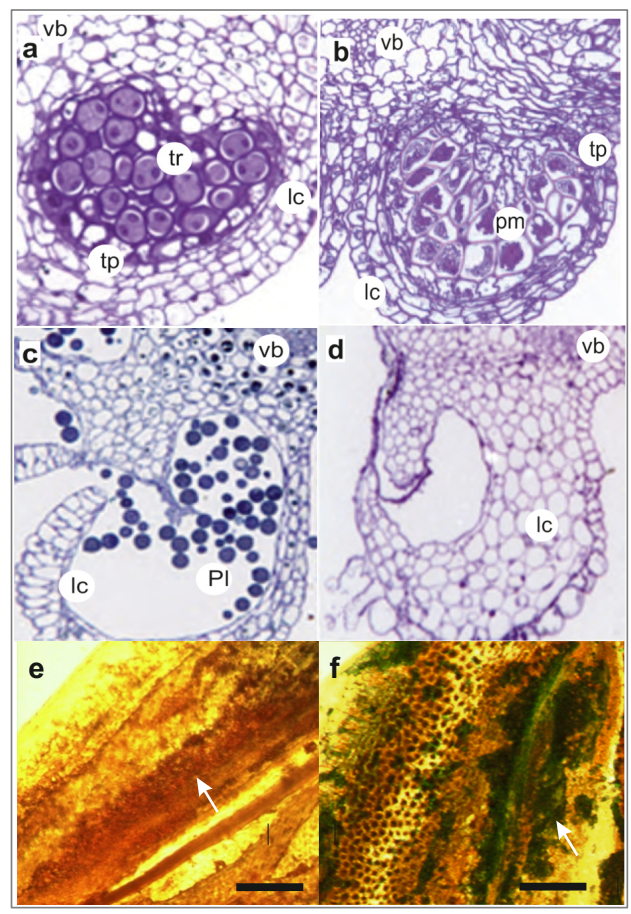

Figure 1. Effects of MHS on anther development and pollen biogenesis in MM.

a. Transverse section through a $3 \mathrm{~mm}$ anther of MM grown under standard conditions $\mathrm{vb}$, tp, and tr indicate vascular bundles, tapetum and tretrads respectively. b. Transverse section through a $3 \mathrm{~mm}$ anther grown under MHS, pm indicates the degraded pollen mother cells. c. Transverse section through mature anthers with developed pollen. Pl, lc vb indicate pollen, locule and vascular bundles respectively. d. Transverse section through mature anthers, grown under MHS. lc vb indicate pollen, locule and vascular bundles respectively. e-f. Longitudinal section through $3 \mathrm{~mm}$ anthers of control and MHS anthers (respectively) stained with Alexander dye. Arrows show pollen with grey colouration indicating degraded pollen tissues. 


\section{Identification of differentially expressed transcripts by cDNA-AFLP}

The aim of this experiment was to investigate the impact of MHS on gene transcription in meiotic anthers in tomato. We used a heat-sensitive tomato cultivar MM and recorded the number of genes affected, the timing of the transcriptional response and the functional classification of the modulated genes. To provide a rapid view on gene expression profiles, a modified cDNA-AFLP protocol was used. The template for the cDNA-AFLP screening was isolated from 3-5 mm anther cones treated with MHS and compared to anther cones from material grown under standard conditions. 92 cDNA-AFLP primer combinations were used to assess gene transcription and this screen lead to the identification of approximately 7300 unique transcript-derived fragments (TDFs) of 100-500 bp. We calculate that around $30 \%$ of the transcriptome of meiotic anthers has been visualised in this screen according to an estimation previously suggested by Cnudde et al. 2006 (Shen et al., 1997).

The cDNA-AFLP analysis showed that the majority of bands exhibit no change in intensity in response to moderate heat stress. Thus, after $30 \mathrm{~h}$ of MHS, approximately $1 \%$ of the examined transcript-derived fragments changed in expression. The most marked transcriptional responses for this heat-sensitive genotype were found at $2 \mathrm{~h}$ after initiating MHS. 96 TDFs showed a clear differential expression pattern. Of these, 41 showed a down-regulated pattern, 26 TDFs were transiently induced, 16 were up-regulated and 13 were transiently repressed. No further significant changes in gene expression were found after 6, 16 or $30 \mathrm{~h}$ of MHS (Figure 2).

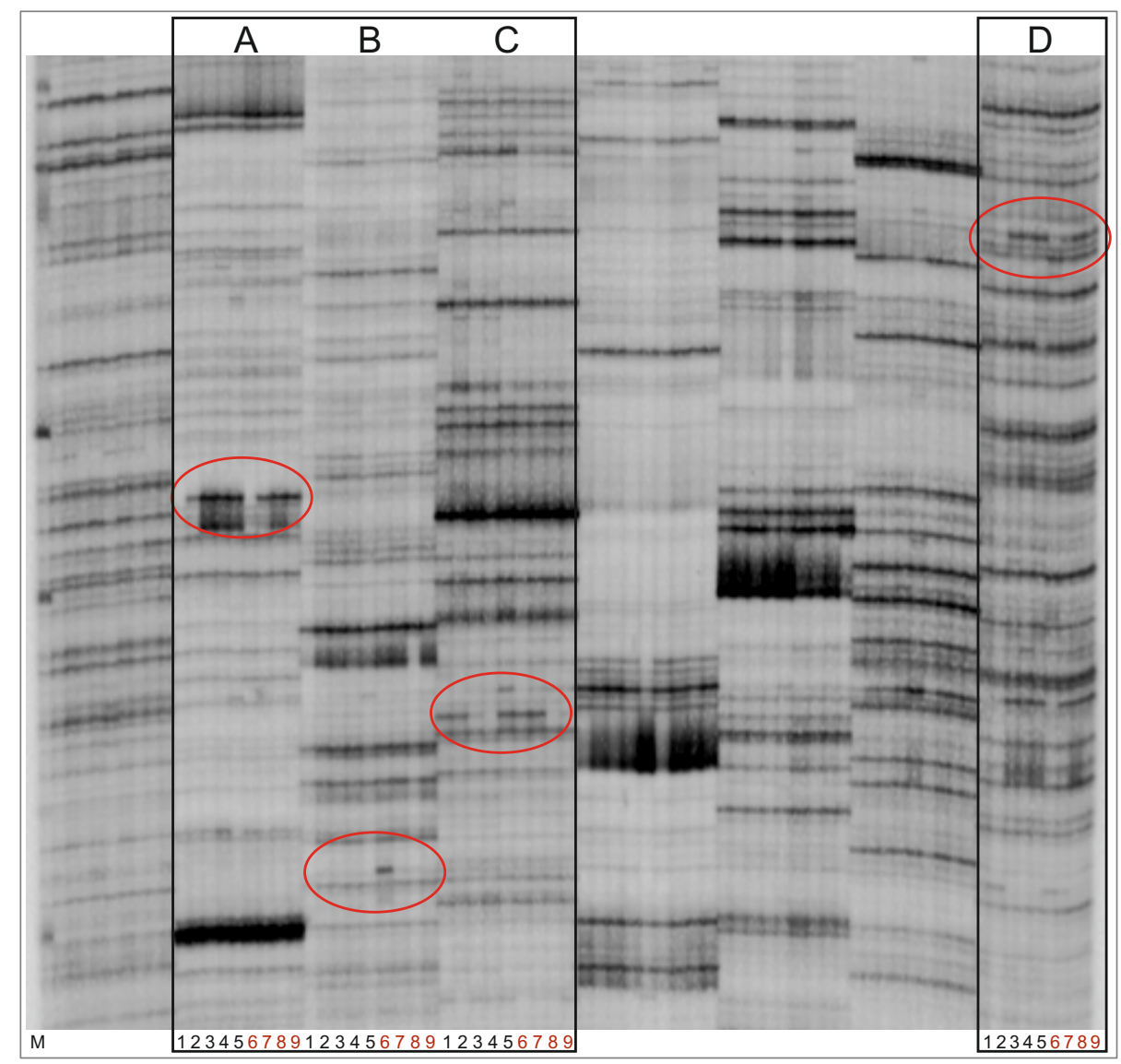

Figure 2. Example of a cDNA-AFLP profile of RNA from meiotic anthers.

Eight primer combinations are shown with each 5 time points of controls (lanes 1-5) and 4 samples from MHS (lanes 6-9 - shown in red). In four panels (A-D) representing four different primer combinations, differentially expressed TDFs are highlighted with red circles 


\section{Moderate heat stress affects different functional categories of genes}

As a next step, a selection of 25 TDFs exhibiting differential gene expression were sequenced in order to gain insight into the processes involved in the response(s) to MHS. Based upon the observed differences in response to MHS and on literature sources, we identified a number of candidate transcripts involved in heat-tolerance.

The putative functions associated with the genes identified by cDNA-AFLP indicate involvement of heat shock, metabolism, antioxidant and development functional classes. Figure 3 summarises the expression patterns of these TDFs and provides the homology-based putative functions of the associated genes. From the tomato Gene Index (DFCI), 14 TDFs showed sequence similarity to stress-related genes from several plant species. After submission to Uniprot databases, these homologies suggested the involvement of metabolism, transport, heat shock as well as oxydoreductive processes. Another 11 fragments showed no homology with known genes. The 25 identified transcripts were all $100 \%$ identical to tomato ESTs identified as tentative consensuses (TCs) and represented by individual sequence assemblies at the DFCI. The rapid modulation of TDFs suggests a short window to initiate protection and detoxification.
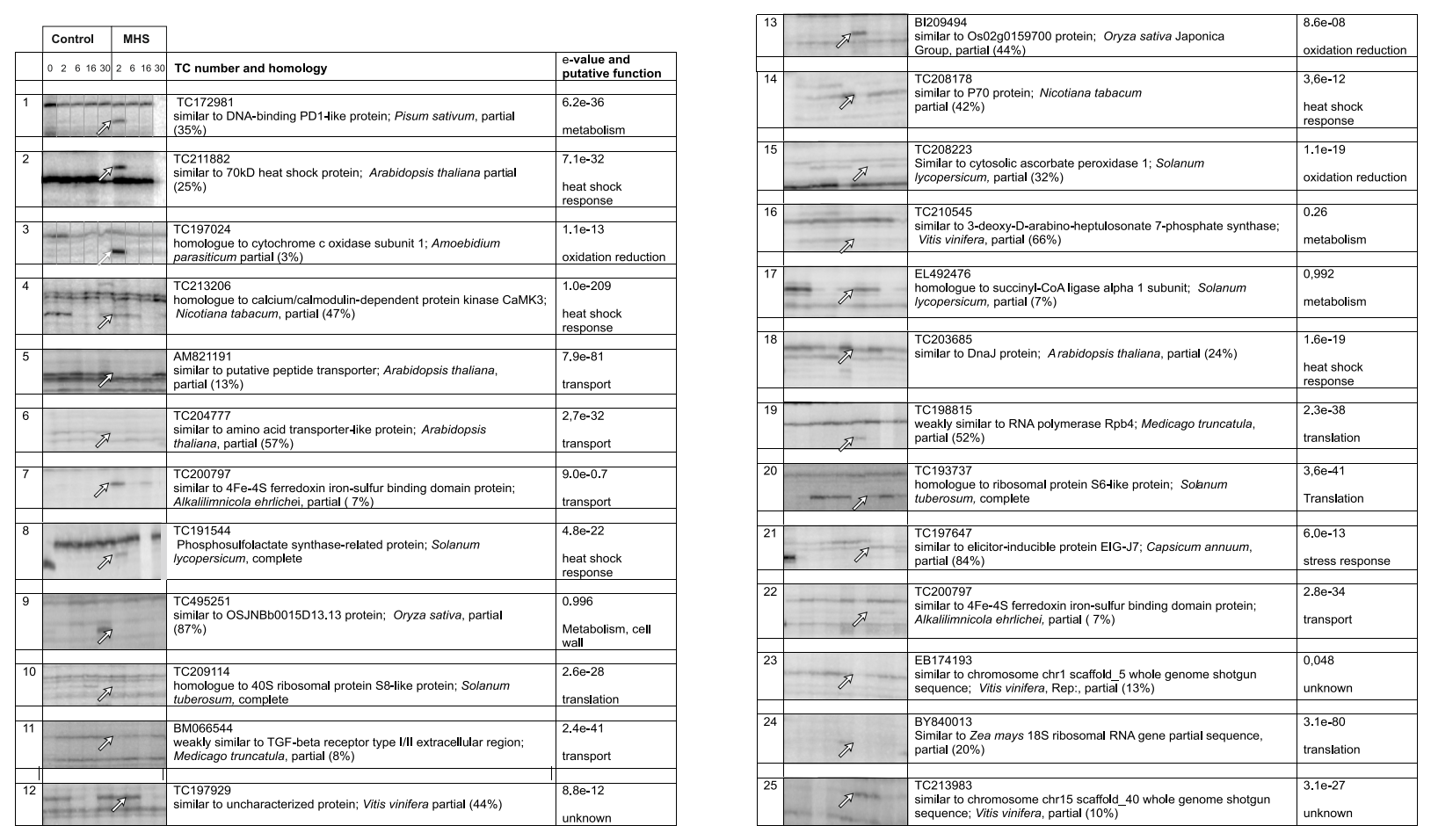

Figure 3. cDNA-AFLP gene expression profiling. Examples of individual cDNA-AFLP profiles are shown with arrows indicating the transcript-derived fragment of interest (TDF). For each lane the sampling times for both the controls and the MHS conditions are shown at the top. Next to each profile, the TC with the highest homology is shown with a brief summary of its putative function. The homology e-value is given in the right column. 


\section{Gene expression and localisation in developing tomato anthers under MHS}

In order to confirm the observed cDNA-AFLP expression profiles, RT-PCR experiments were carried out on MM meiotic anther RNA for a set of chosen transcripts with defined profiles, from different functional classes. TC211882 and TC191544 are heat shock genes, TC197647 represents a stress inducible protein while TC213983 represents an unknown transcript with a differentially expressed pattern. The RT-PCR profiles confirmed the induction or repression upon moderate heat stress as depicted by the AFLP analysis (Figure 4).

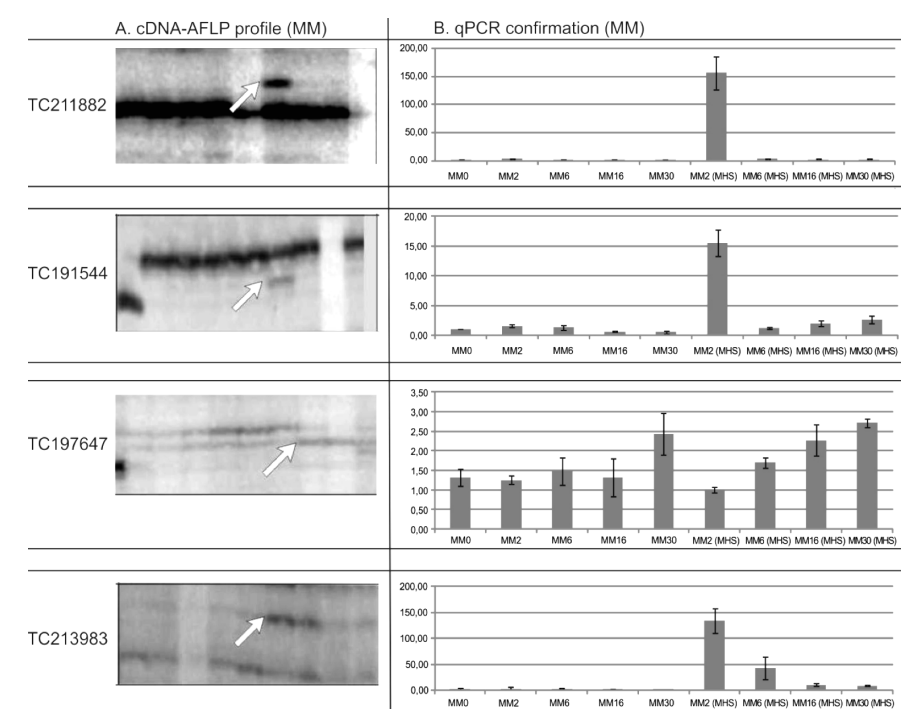

Figure 4. Validation of the cDNA-AFLP profiling with RT-PCR Four TDFs (left panels; A) corresponding to TC211882, TC191544, TC197647 and TC213983 were verified with quantitative RT-PCR in the genotype Money Maker (MM; right panels; B) at time points $0 \mathrm{~h}, 2 \mathrm{~h}, 6 \mathrm{~h}, 16 \mathrm{~h}$ and $30 \mathrm{~h}$ under standard growth and MHS conditions. The bars represent expression values derived from the delta $\mathrm{CT}$ values and error bars are given as standard deviation.

To detect if the transcripts are anther specific, the four TDFs were also analysed by RT-PCR for tissue specificity (Figure 5). All transcripts are predominantly expressed in reproductive organs, even though moderate expression levels are also detected in other plant tissues such as leaf, stem, seedling and root..

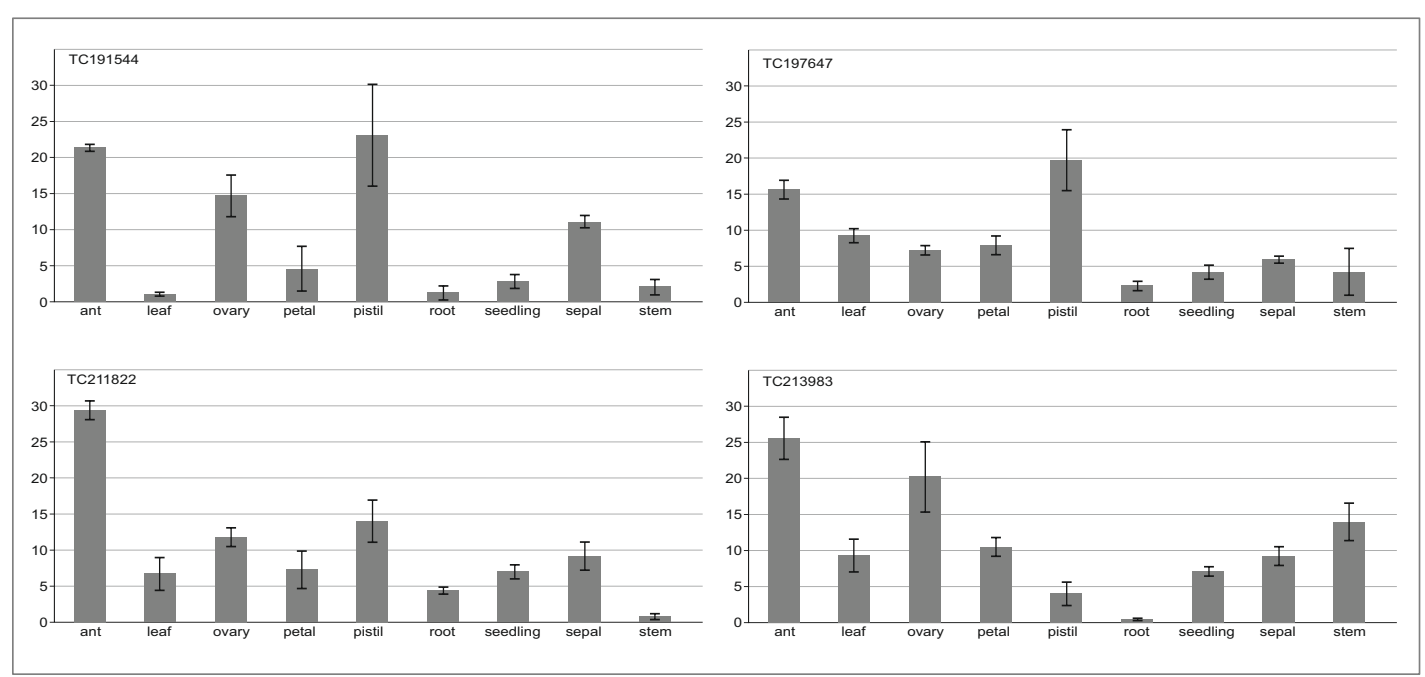

Figure 5. Tissue specificity of selected TDFs analysed by RT-PCR. Relative levels of RNA are shown from various tissues as indicated below the bars. The bars represent expression values derived from the delta CT values and error bars are given as standard deviation. 
To localise the expression of the chosen transcripts, a set of in situ hybridisations were performed on meiotic anthers (Figure 6). As described above TCs 211822 and 191544 are both involved in protection from heat shock. TC211822 is a chaperone and TC191544 is involved in the maintenance of thermotolerance. These two heat shock genes appear to localise primarily to the tapetum and interlocular connective tissue, while the stress inducible and the unknown gene appear to also localise to the developing microspores. Although TC197647 putative function cannot directly be linked with heat shock as an elicitor inducible protein with lipase function, the in situ hybridisation shows expression specificity for both tapetum and developing pollen cells. Also TC213983 (unknown function) shows specificity for pollen and tapetum, however at a much lower level compared to the other tested genes.

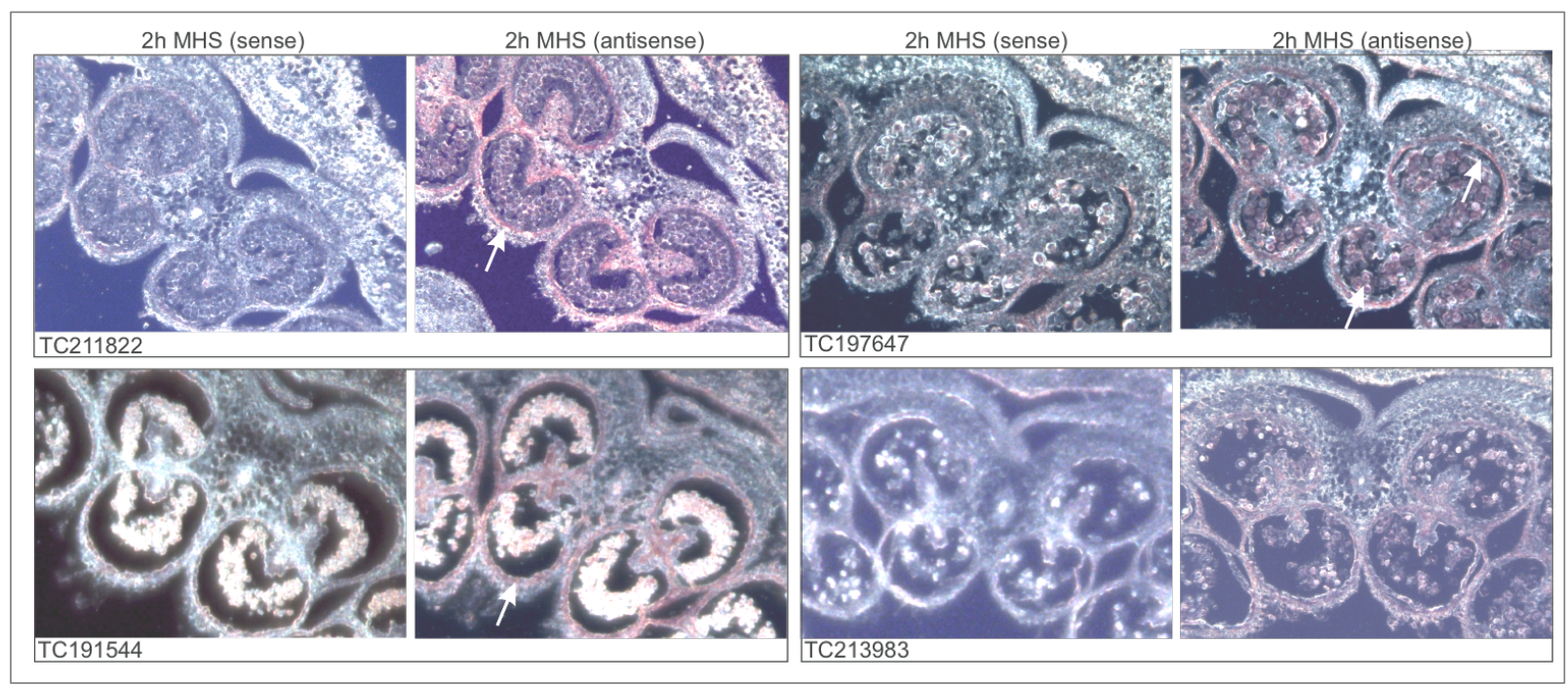

Figure 6. In situ mRNA localisation using the 4 TDFs described above. Sections of heat stressed meiotic anthers were hybridized with sense (left) and anti-sense (right). Accumulation of the heat stress induced transcripts was detected particularly in the tapetum and/or in developing microspores (white arrows).

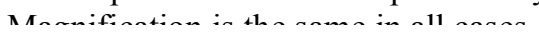

To validate the expression data found in the MM genotype we chose two additional genotypes that contrast strongly in their response to temperature stress. Both Falco Rosso (FR) and Heat Set 1 (HS1) are commercial hybrids (Nunhems B.V.). FR is a heat sensitive genotype while HS1 is a heat tolerant genotype according to unpublished observations communicated by the breeders. These lines and their transcriptome responses to heat have been described in more detail (Bita et al., 2011). To compare the transcription between MM and the two contrasting genotypes, RT-PCR was carried out with the same TDF derived sequences that were analysed in MM (Figure 4). The TDFs corresponding to a chaperon (TC211882) shows a similar response compared to MM. However, in the tolerant genotype HS1 there is a higher expression at time point $2(2 \mathrm{~h})$. TC191544 has a function in the maintenance of thermotolerance and here there is an increased response to high temperature in the HS1 genotype. It is interesting to note that expression of TC191544 is relatively high at time point 0 and continues to increase up to the last time point in HS1, which is consistent with its function. TC197647 shows a similar expression profile in the sensitive FR genotype as in MM. However in the HS1 tolerant genotype there is a higher expression at time point 2 compared to the other two time points. TC213983 shows a similar profile in both genotypes, however with a reduced response under MHS. 


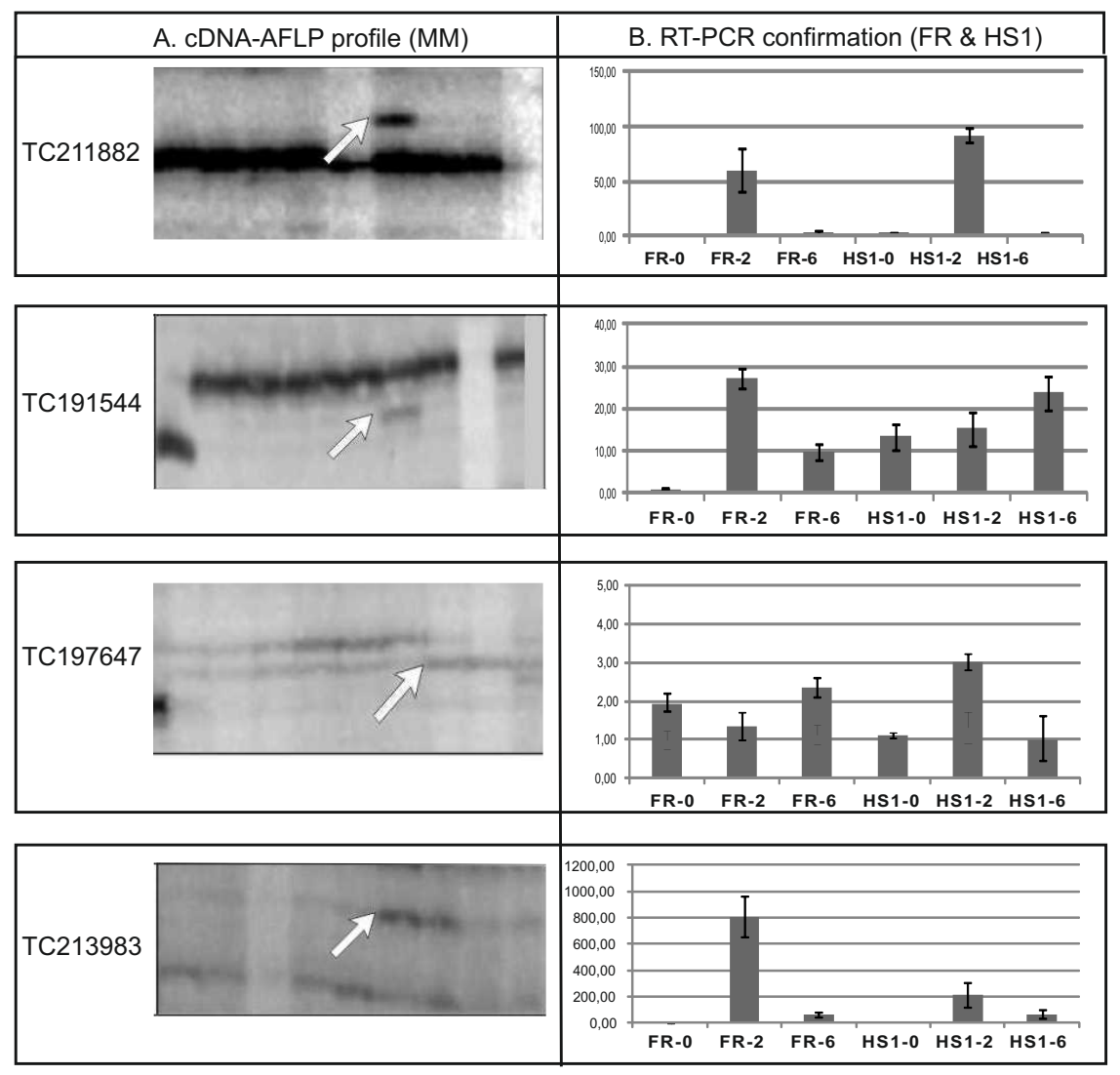

Figure 7. Comparison of cDNA-AFLP profiles of four genes compared to RT-PCRs in two genotypes with contrasting heat tolerance. Four TDFs (left panel, at time points $0 \mathrm{~h}, 2 \mathrm{~h}, 6 \mathrm{~h}, 16 \mathrm{~h}$ and $30 \mathrm{~h}$, cf figure 4) corresponding to TC211882, TC191544, TC197647 and TC213983 were verified with quantitative RT-PCR in the sensitive genotype FR and tolerant genotype HS1 (right panels) at time points: $0 \mathrm{~h}, 2 \mathrm{~h}, 6 \mathrm{~h}$ under MHS conditions. The bars represent expression values derived from the delta $\mathrm{CT}$ values and error bars are given as standard deviation.

\section{Conclusion}

We conclude that the cDNA-AFLP experiments showed the effect of MHS on the meiotic anther transcriptome to be visible as of $2 \mathrm{~h}$ of treatment with different effects in heat sensitive and tolerant genotypes. In response to MHS, a differential modulation of approximately $1 \%$ of the anther transcriptome was observed in the sensitive MM genotype. The identified genes are significantly changed in expression, indicating that meiotic anther development is sensitive to moderate heat stress as early as $2 \mathrm{~h}$ of MHS. Most of the isolated and sequenced TDFs that are transiently upregulated at $2 \mathrm{~h}$ belong to the protection and antioxidant classes, while some of the downregulated TDFs on the entire treatment appear to be involved in metabolic processes. Such modulation has been described in the microspores of other tomato heat sensitive genotypes as well, where reactive oxygen species scavengers and heat shock proteins also accumulate following a heat stress treatment (Zinn et al., 2010). The extensive localization of heat shock proteins to both microspores and the tapetum may be another indication of sensitivity to heat of our genotype, as a heat tolerant genotype showed heat shock protein accumulation during heat stress only in microspores and not in the tapetum (Giorno et al., 2010).

cDNA-AFLP remains one of the methods of choice for analysing differential gene expression. It provides a particular advantage in biological systems where little sequence information is available and/or technical resources are limited. Amongst the open architecture technologies, cDNA-AFLP is a 
good alternative for the discovery of novel, lowly expressed genes, which can help to ascribe such genes towards their characterization and linking to particular phenotypes and biochemical pathways. The development of technologies for detection of specific sequences by tagging has developed rapidly along with the development of new sequencing strategies.

The possibility of using AFLP type tags on cDNA fragments derived from restriction digests in these technologies opens up the possibility of not only identifying very large numbers of expressed genes but also to retrieve SNP data in addition to the expression level based on abundance of specific TDFs. Although the technologies used for cDNA-AFLP are changing rapidly, the principles underlying this anonymous transcript profiling method continue to be highly relevant today. 


\section{Chapter Appendix}

Primers used to validate of gene expression by real-time PCR (RT-PCR)

\begin{tabular}{|l|l|l|}
\hline TC211882 & TC211882F & 5'-CGAGATTGCTGAATACATAAT \\
\hline & TC211882R & 5'-TGTTTTATATTTGCCAGAGAA \\
\hline TC191544 & TC $191544 \mathrm{~F}$ & $5^{\prime}$-AGTGGGCTCAGGGCTAAG \\
\hline & TC $191544 \mathrm{R}$ & $5^{\prime}$-CCTCTGAGATGGTTGAAGATGT \\
\hline TC197647 & TC $197647 \mathrm{~F}$ & $5^{\prime}$-AGGTTCAGGTCATGGATGG \\
\hline & TC $197647 \mathrm{R}$ & $5^{\prime}$-GCTGACTGCCATTAGAGATC \\
\hline TC213983 & TC $213983 \mathrm{~F}$ & $5^{\prime}$-CCGGTGGATGGAAGTAAAGC \\
\hline & TC $213983 \mathrm{R}$ & 5'-GTCTAGCAGATGACCACAACAT \\
\hline
\end{tabular}

Primer sequences used for the in situ hybridisation reactions

\begin{tabular}{|l|l|l|}
\hline TC211882 & TC $211882 \mathrm{sF}$ & 5'-CGGAGGTATACCCAAAGC \\
\hline & TC $211882 \mathrm{sR}$ & 5`-CATCGCCTTCGTAAACCC \\
\hline TC191544 & TC $191544 \mathrm{sF}$ & 5`TGAAAGCTTCTTCAAATCATA \\
\hline & TC $191544 \mathrm{sR}$ & 5`AATTGCTTACATTCCTCAATA \\
\hline TC197647 & TC $197647 \mathrm{sF}$ & 5`-TTCAGGTCATGGATGGTA \\
\hline & TC $197647 \mathrm{~s} \mathrm{R}$ & 5`TATAAGCGAGCACAGTAAAA \\
\hline TC213983 & TC $213983 \mathrm{sF}$ & 5`AATTAGAAGAGCATGGAGATG \\
\hline & TC $213983 \mathrm{sR}$ & 5`-ACCTCAATTGTTTCTGATTCC \\
\hline
\end{tabular}




\section{References}

Arenas-Huertero, F., Arroyo, A., Zhou, L., Sheen, J. and Leon, P. (2000) Analysis of Arabidopsis glucose insensitive mutants, gin 5 and gin6, reveals a central role of the plant hormone ABA in the regulation of plant vegetative development by sugar. Genes \& development 14, 20852096.

Bachem, C.W.B., van der Hoeven, R.S., de Bruijn, S.M., Vreugdenhil, D., Zabeau, M. and Visser, R.G. (1996) Visualization of differential gene expression using a novel method of RNA fingerprinting based on AFLP: analysis of gene expression during potato tuber development. The Plant Journal 9, 745-753.

Bartels, D. (2001) Targeting detoxification pathways: an efficient approach to obtain plants with multiple stress tolerance? Trends in plant science 6, 284-286.

Bedinger, P. (1992) The remarkable biology of pollen. Plant Cell 4, 879.

Bita, C.E., Zenoni, S., Vriezen, W.H., Mariani, C., Pezzotti, M. and Gerats, T. (2011) Temperature stress differentially modulates transcription in meiotic anthers of heat-tolerant and heatsensitive tomato plants. BMC genomics 12, 384.

Bolouri-Moghaddam, M.R., Le Roy, K., Xiang, L., Rolland, F. and Van den Ende, W. (2010) Sugar signalling and antioxidant network connections in plant cells. Febs Journal 277, 2022-2037.

Bray, E.A., Bailey-Serres, J. and Weretilnyk, E. (2000) Responses to abiotic stresses. Biochemistry and molecular biology of plants, 1158-1203.

Chinnusamy, V., Gong, Z.Z. and Zhu, J.K. (2008) Abscisic acid-mediated epigenetic processes in plant development and stress responses. Journal of integrative plant biology 50, 1187-1195.

Chinnusamy, V., Schumaker, K. and Zhu, J.K. (2004) Molecular genetic perspectives on cross-talk and specificity in abiotic stress signalling in plants. Journal of experimental botany 55, 225236.

Ciarmiello, L.F., Woodrow, P., Fuggi, A., Pontecorvo, G. and Carillo, P. (2011) Plant genes for abiotic stress.

Endo, M., Tsuchiya, T., Hamada, K., Kawamura, S., Yano, K., Ohshima, M., Higashitani, A., Watanabe, M. and Kawagishi-Kobayashi, M. (2009) High temperatures cause male sterility in rice plants with transcriptional alterations during pollen development. Plant and cell physiology 50, 1911-1922.

Feder, M.E. and Hofmann, G.E. (1999) Heat-shock proteins, molecular chaperones, and the stress response: evolutionary and ecological physiology. Annual review of physiology 61, 243-282.

Frank, G., Pressman, E., Ophir, R., Althan, L., Shaked, R., Freedman, M., Shen, S. and Firon, N. (2009) Transcriptional profiling of maturing tomato (Solanum lycopersicum L.) microspores reveals the involvement of heat shock proteins, ROS scavengers, hormones, and sugars in the heat stress response. Journal of experimental botany 60, 3891-3908.

Gibson, G. (2003) Microarray analysis: genome-scale hypothesis scanning. PLoS Biol 1, E15.

Giorno, F., Wolters-Arts, M., Grillo, S., Scharf, K.D., Vriezen, W.H. and Mariani, C. (2010) Developmental and heat stress-regulated expression of HsfA2 and small heat shock proteins in tomato anthers. Journal of experimental botany 61, 453-462.

Hall, A. (2001) Crop developmental responses to temperature, photoperiod, and light quality. Crop Response to Environment. Boca Raton, FL: CRC, 83-87.

Hedhly, A. (2011) Sensitivity of flowering plant gametophytes to temperature fluctuations. Environmental and Experimental Botany 74, 9-16.

Hey, S.J., Byrne, E. and Halford, N.G. (2010) The interface between metabolic and stress signalling. Annals of botany 105, 197-203.

Kaya, C., sonmez, O., Aydemir, S., Ashraf, M. and Dikilitas, M. (2012) Exogenous application of mannitol and thiourea regulates plant growth and oxidative stress responses in salt-stressed maize (Zea mays L.). Journal of Plant Interactions, 1-8.

Kim, S.Y., Hong, C.B. and Lee, I. (2001) Heat shock stress causes stage-specific male sterility in Arabidopsis thaliana. Journal of plant research 114, 301-307.

Larkindale, J. and Knight, M.R. (2002) Protection against heat stress-induced oxidative damage in arabidopsis involves calcium, abscisic acid, ethylene, and salicylic acid. Plant physiology $\mathbf{1 2 8}$, 682-695. 
Levy, A., Rabinowitch, H. and Kedar, N. (1978) Morphological and physiological characters affecting flower drop and fruit set of tomatoes at high temperatures. Euphytica 27, 211-218.

Mittler, R. (2006) Abiotic stress, the field environment and stress combination. Trends in plant science 11, 15-19.

Peet, M.M. (1998) Greenhouse crop stress management. International Symposium on Growing Media and Hydroponics, Vols I and Ii, 643-654.

Peet, M.M., Sato, S. and Gardner, R.G. (1998) Comparing heat stress effects on male-fertile and malesterile tomatoes. Plant Cell and Environment 21, 225-231.

Peng, Y.B., Zou, C., Wang, D.H., Gong, H.Q., Xu, Z.H. and Bai, S.N. (2006) Preferential localization of abscisic acid in primordial and nursing cells of reproductive organs of Arabidopsis and cucumber. New Phytologist 170, 459-466.

Peshev, D. and Van den Ende, W. (2012) Sugars as antioxidants in plants. Crop improvement under adverse conditions, 285-308.

Sato, S., Kamiyama, M., Iwata, T., Makita, N., Furukawa, H. and Ikeda, H. (2006) Moderate increase of mean daily temperature adversely affects fruit set of Lycopersicon esculentum by disrupting specific physiological processes in male reproductive development. Annals of botany $97,731-738$.

Sato, S., Peet, M.M. and Gardner, R.G. (2004) Altered flower retention and developmental patterns in nine tomato cultivars under elevated temperature. Scientia Horticulturae 101, 95-101.

Shen, B., Jensen, R.G. and Bohnert, H.J. (1997) Mannitol protects against oxidation by hydroxyl radicals. Plant physiology 115, 527-532.

Sreenivasulu, N., Harshavardhan, V.T., Govind, G., Seiler, C. and Kohli, A. (2012) Contrapuntal role of ABA: Does it mediate stress tolerance or plant growth retardation under long-term drought stress? Gene 506, 265-273.

Suzuki, K., Takeda, H., Tsukaguchi, T. and Egawa, Y. (2001) Ultrastructural study on degeneration of tapetum in anther of snap bean (Phaseolus vulgaris L.) under heat stress. Sexual Plant Reproduction 13, 293-299.

Vos, P., Hogers, R., Bleeker, M., Reijans, M., van de Lee, T., Hornes, M., Frijters, A., Pot, J., Pelman, J., Kuiper, M. and Zabeau, M. (1995) AFLP: a new technique for DNA fingerprinting. Nucleic Acids Res 23, 4407-4414.

Walley, J.W. and Dehesh, K. (2010) Molecular Mechanisms Regulating Rapid Stress Signaling Networks in Arabidopsis. J Integr Plant Biol 52, 354-359.

Wilkinson, S. and Davies, W.J. (2010) Drought, ozone, ABA and ethylene: new insights from cell to plant to community. Plant Cell and Environment 33, 510-525.

Zinn, K.E., Tunc-Ozdemir, M. and Harper, J.F. (2010) Temperature stress and plant sexual reproduction: uncovering the weakest links. Journal of experimental botany 61, 1959-1968. 


\section{Chapter 4}

\section{Anther transcriptome profiling in tolerant and sensitive tomato genotypes under moderate heat stress}

Published as:

Craita Elena Bita, Sara Zenoni, Wim Vriezen, Celestina Mariani, Mario Pezzotti, Tom Gerats (2011) Temperature stress differentially modulates transcription in meiotic anthers of heat-tolerant and heat sensitive tomato plants. BMC Genomics 12: 384 


\begin{abstract}
Tolerance to heat stress can be achieved either by constitutive gene expression or by rapidly induced changes in gene expression, leading to protective responses, which minimize potential damage. Among general stress tolerance mechanisms, stress proteins, osmo-protectants, free-radical scavengers, ion transporters and factors involved in signalling cascades and transcriptional control are essential to counteract stress effects. We used a Combimatrix microarray analysis to profile the early response of tomato meiotic anthers transcriptome to moderate heat stress conditions $\left(32^{\circ} \mathrm{C}\right)$ in a heattolerant tomato genotype and compared it to a heat-sensitive tomato genotype. The microarray analysis revealed that about $1 \%$ of the genes have a significant modulation in expression after $2 \mathrm{~h}$ of moderate heat stress and that the heat-tolerant genotype exhibits fewer transcriptional changes than the heat-sensitive genotype upon moderate heat stress. Moreover, the heat-tolerant genotype also shows a different constitutive gene expression make-up compared to the heat-sensitive one, indicating differences in genetic adaptation to increased temperatures. In the heat-tolerant genotype, the majority of changes in gene expression is represented by up-regulation, while in the heat-sensitive genotype there is a general trend to down-regulate gene expression, soon after MHS. The putative functions associated with the genes identified by microarray profiling indicate involvement of heat shock, antioxidant, metabolic, and cell development pathways. Based upon the observed differences in response to MHS we identified a number of candidate transcripts involved in heat-tolerance and confirmed their expression pattern in different tomato genotypes with contrasting responses to heat.
\end{abstract}




\section{Introduction}

Abiotic stresses resulting from extreme temperatures, drought, flooding or chemical toxicity poses serious threats to agriculture and only a rapid adaptation or built-in tolerance mechanisms can provide relative security for further plant development and, more importantly fruit-set. Gamete development in angiosperms takes place within two floral organs, the male stamen and the female pistil (Drews and Goldberg, 1989; Goldberg, 1988). The developmental pathway for a pollen grain starts with the separation of the reproductive tissues of the anther, continues with meiosis of the pollen mother cell, followed by mitosis and microspore maturation that results in the mature pollen grain. After initiation, the highly specialized anther tissues will acquire non-reproductive (e.g., the tapetum for support, stomium for dehiscence) or reproductive functions (egg cell and pollen - formation). Both tapetum and microspore development are essential for male fertility, as documented by numerous studies on male sterile mutants (Aarts et al., 1997; Ariizumi et al., 2003; Chen and McCormick, 1996; Hulskamp et al., 1997; Kaul, 1988; Taylor et al., 1998; van der Meer et al., 1992). In Petunia, a cDNA-AFLP study revealed that during meiosis in anthers under standard conditions, besides the typical meiotic genes, other genes are also important for the development of the pollen grains. Thus, during meiosis, tapetum differentiation genes, serine-proteinases, hormone precursors, genes involved in cell wall biosynthesis and even ribonucleases and polyamine precursors are modulated in expression. RNA levels decrease, mitochondria and plastids degenerate, while DNA, protein, RNA, and cell wall synthesis are maintained (Cnudde et al., 2006).

Tomato (Solanum lycopersicum L.) displays marked responses to heat, similar to other crop species including pepper, potato, melon, cowpea, wheat, common bean, rice and barley (Abiko et al., 2005; Lindquist and Craig, 1988; Porch and Jahn, 2001). Hot summers in many agricultural regions can negatively affect the vegetative and reproductive growth phases of such crops (Pressman et al., 2002) and can result in up to $70 \%$ tomato harvest losses (Sato et al., 2004). However, heat stress has diverse specific effects depending on the genotype. Physiological observations both under field and greenhouse conditions reveal a variable degree of tolerance between different genotypes. A wide range of heat stress phenotypes has been described (Kamel et al., 2010; Sato and Peet, 2005). For example, of five tomato cultivars grown under MHS conditions $\left(32^{\circ} \mathrm{C}\right.$ day and $26^{\circ} \mathrm{C}$ night), only one set fruit (Sato et al., 2000). The differences in pollen grain development among the tolerant genotypes are considered the most critical factors to determine fruit set under heat stress. Furthermore, a comparison of the effects of heat stress on a heat-tolerant and a heat-sensitive tomato cultivar showed that temperature stress affected mainly the development of pollen grains, where reduced viability was more pronounced in the heat-sensitive cultivar (Levy et al., 1978).

Under heat stress, it is the pollen grain development and particularly meiosis that shows the highest susceptibility, followed by germination and pollen tube growth, which in more severe cases can also be significantly affected (Kakani et al., 2005; Kim et al., 2001; Sato et al., 2002b). Recently, an analysis of maturing tomato microspores (mitotic anthers) exposed to heat-shock conditions has shown no differential gene expression between heat-tolerant and heat-sensitive genotypes but it seems that the capacity for thermotolerance may be achieved by modulating the expression levels of such 'responsive' genes prior to heat stress exposure. (Frank et al., 2009)

Microarray analysis is used to survey the expression of thousands of genes in a single experiment. Applied creatively, that can be used to test as well as generate new hypotheses. Microarrays are simply a method for visualizing which genes are likely to be used in a particular tissue at a particular time under a particular set of conditions. All microarray experiments rely on the core principle that transcript abundance can be deduced by measuring the amount of hybridization of labeled RNA to a complementary probe. In microarray analysis it is the comparison of gene expression profiles that is usually of most interest: if a difference in transcript abundance is observed between two or more conditions, it is natural to infer that the difference might point to an interesting 
biological phenomenon (Gibson, 2003).Our transcriptomic analysis was performed on the newly developed CombiMatrix platform at the University of Verona, Italy, on a tomato chip carrying 20200 specific probes in quadruplicates from assembly of Tentative Consensus of the last Tomato Gene Index (LeGI). The CombiMatrix CustomArray TM technology is characterized by an exclusive in situ oligo (up to 40 mers) synthesis driven by electrochemistry and by the reusability of the same microarray chip, all factors that confer high flexibility to the system and reduce remarkably the costs of microarray analysis (CombiMatrix Diagnostics; http://www.combimatrix.com, Functional Genomic Center; http://ddlab.sci.univr.it/FunctionalGenomics).

In this chapter we present an analysis of the fluctuations in gene expression in developing meiotic anthers (the first and most sensitive anther developmental stage) in response to moderate heat stress (MHS) of tolerant and sensitive tomato genotypes. Assuming the protective mechanisms are initiated shortly after the commencement of the stress period, we decided to study gene expression changes in the first $6 \mathrm{~h}$ of MHS. The Combimatrix microarray technology was applied to obtain a general overview of molecular mechanisms that participate in the response to MHS of anthers from a heat-tolerant and a heat-sensitive genotype of tomato. Accordingly, we describe a set of genes and candidate pathways that open up the possibility of investigating and modulating male heat tolerance in tomato and other commercially important Solanaceae crops.

\section{Materials and Methods}

\section{Plant material and heat stress conditions}

A tomato heat-tolerant, Heat Set1 (HS1) and a tomato heat-sensitive genotype Falcorosso (FR) were used. FR, obtained from Nunhems Netherlands BV (the Netherlands), is a commercial variety and HS1, also provided by Nunhems BV, is a variety selected for good fruit set in field conditions where temperatures are higher than $38^{\circ} \mathrm{C}$. Seeds were germinated in potting compost in trays and transferred after two weeks to a growth chamber under standard temperature (ST) conditions, with a 16-h light $\left(26^{\circ} \mathrm{C}\right) / 8$-h dark $\left(18^{\circ} \mathrm{C}\right)$ cycle. Fluorescent and incandescent lighting provided a photosynthetic photon flux density of $450-500 \mu \mathrm{mol} \mathrm{m} \mathrm{sec}^{-1}$. Six-week-old plants were heat stressed by raising the temperature to a regime of $\mathrm{MHS}\left(32^{\circ} \mathrm{C} / 26^{\circ} \mathrm{C}\right.$; day/night). The heat treatment was initiated by progressively increasing the temperature from 25 to $32^{\circ}$ over half an hour period, and samples were collected at $0,2,6,16$ or $30 \mathrm{~h}$ of MHS. In order to describe the dynamics of transcriptional responses of tomato developing meiotic anthers to MHS, the temperature range for the experiment was chosen based on agronomically relevant temperatures shown to have a significant effect on pollen viability (Sato et al., 2002a), rather than using classical heat shock conditions of $42^{\circ}-45^{\circ} \mathrm{C}$ (Frank et al., 2009). Heat stress was applied to whole plants in the growth chamber, under the same light conditions as stated above.

\section{Anther isolation and RNA extraction}

We focused our analysis of gene expression on whole meiotic anther cones isolated from flower buds of plants that were exposed to MHS $\left(32{ }^{\circ} \mathrm{C} / 26{ }^{\circ} \mathrm{C}\right.$, day/night) for up to $30 \mathrm{~h}$, and compared this to standard temperatures $\left(26^{\circ} \mathrm{C} / 18{ }^{\circ} \mathrm{C}\right.$ day/night). For the microarray experiment, a pool of six meiotic flower buds from 3 plants were harvested (according to (Brukhin et al., 2003)). Pilot experiments indicated the third and fourth flower clusters to give the most consistent phenotypic response to moderate heat stress (data not shown). From these, anther cones ranging in size from $2-4 \mathrm{~mm}$ were isolated, immediately frozen in liquid $\mathrm{N}_{2}$ and pooled for RNA extraction. Tissues were homogenized using glass rods and RNA was extracted using a Plant RNA isolation kit (Plant RNeasy Mini Kit, Qiagen Benelux BV, Venlo the Netherlands) according to the manufacturer's recommendations. 


\section{Microarray hybridization and data analysis}

The $90 \mathrm{~K}$ Custom TomatoArray 1.0 chip (Combimatrix microarray platform, http://ddlab.sci.univr.it/FunctionalGenomics/) consists of 20200 unique probes derived from Solanum lycopersicum transcripts and various controls, and was produced by the Plant Functional Genomics Center, University of Verona. The gene specific probes (oligonucleotide of 35-40-mer) randomly distributed in quadruplicate across the array, were designed using the program design OligoArray 2.1 (Levy et al., 1977). The sequences represented on the chip correspond to 20115 S. lycopersicum TCs (Gene Index Release 11.0, 21 June, 2006) and to 85 technical controls (negative and positive spiking controls). From the 20115 tomato probes represented on the chip, 17018 probes were found to be overall expressed above background and consistent among the technical repeats within each chip. The quality of the biological replicates was evaluated by Pearson coefficient, which ranged from 0.87 to 0.98. For the microarray experiment, 3 biological replicas for each sample were used. Material was harvested at $0 \mathrm{~h}$ (ST) and at $2 \mathrm{~h}$ and $6 \mathrm{~h}$ from both genotypes (FR and HS1). Amino allyl-RNA synthesis (aRNA) and labelling with the Alexa647 dye were performed from $1 \mu \mathrm{g}$ of total RNA, with the "SuperScript TM Indirect RNA Amplification System" (Invitrogen, Carlsbad, CA) according to manufacturer's recommendations. $4 \mu \mathrm{g}$ of labelled RNA, with DOL value ranging from 2.0 to 3.0, were first fragmented and hybridized to the array as indicated by the manufacturer (www.combimatrix.com) Pre-hybridization, hybridization, washings and imaging were performed according to the protocols given by CombiMatrix. The array was scanned with a ScanArray 4000XL microarray scanner (Perkin Elmer, Waltham, MA USA). Tiff images were exported to the Microarray Imager 5.8 (Combimatrix) for the densitometry analysis of the spots. The microarray data were normalized by median scaling and the quality of the biological replicates was assessed by means of Pearson's coefficient. Only those genes that were up or down-regulated at least twofold, relative to the average of the three negative controls, for at least one time point, were included in the analysis. The two-fold change in expression as threshold for consideration is a convention employed in various transcript profiling studies (Frank et al., 2009) and therefore is used here to assess the findings from the presented work in comparison with those of earlier reports. All microarray expression data are available at GEO under the series entry GSE24805. The resulting set of significantly changed expression values has been taken into further analysis with paired, unpaired and multiclass SAM analysis, to detect the effect of 'treatment' on expression levels. Genes with similar expression patterns were grouped according to a hierarchical clustering algorithm using Euclidean Distance coefficients.

The paired and unpaired SAM statistical analyses were carried out to determine the significant differences in gene expression between two time-points for each genotype and the significant differences in gene expression between individual time-points observed in both genotypes, respectively. These analyses were performed by T-Mev with a FDR=5\%. For each analysis, a new input dataset was created by filtering the data of the two samples that were compared on the basis of the C.V. values.

In order to determine the significant differences in gene expression accumulated over the entire duration of the experiment all data were filtered on the basis of the $\mathrm{CV}$ value $(\mathrm{CV}<0.5)$ among spot replicates present on the chip and the restricted dataset obtained, of 17018 genes, was suited to run a multiclass comparison method of Significance Analysis of Microarray (SAM), with false discovery rate $(\mathrm{FDR})=5 \%$ (Tusher et al., 2001).

The main components of the changes in gene expression in response to MHS were determined using the set of differentially expressed genes obtained by SAM multiclass analysis, by performing an O2PLS analysis with SIMCA P+ (Umetrics, Umea, Sweden). The unique property of O2PLS is its capacity to identify joint variation between two datasets, while acknowledging also systematic variation that is unique to each dataset. (Wold et al, 2001). The expression profile clustering for each 
genotype was carried out with the Hierarchical Clustering (HCL) method with Euclidean Distance using T-Mev software (Version 4.3).

The Self Ordering Map (SOM) analysis to detect major expression patterns was performed using Pearson's uncentred (absolute) statistic with 2000 iterations (dimension 6: 3X and 2Y). According to the First Order optimisation Method (FOM) analysis, the most representative number of clusters is 6 .

\section{Validation of gene expression by RT-PCR}

Total DNA-free RNA was isolated from tomato anthers ( $\mathrm{n}>5$ ) using a RNA isolation kit (Qiagen RNeasy mini kit with the RNase-Free DNase Set, Qiagen). A PCR reaction with RNA- and DNAspecific primers (Supplementary Materials) based on an intron in the S. lycopersicum actin gene Tom51 (GenBank accession number U60481) was performed to ensure the absence of contaminating genomic DNA. Total DNA-free RNA $(1 \mu \mathrm{g})$ was used for cDNA synthesis (iScriptTM cDNA Synthesis Kit; Bio-Rad Laboratories, Hercules, CA, USA) in a total volume of $25 \mu 1$. PCR reactions were carried out in $25 \mu \mathrm{l}$ containing $0.125 \mu \mathrm{l}$ of cDNA synthesis reaction mixture, $400 \mathrm{~nm}$ of each primer and $12.5 \mu \mathrm{l}$ of iQ SYBR Green Supermix (Bio-Rad Laboratories). PCRs were performed in a 96-well Bio-Rad iCycler (Bio-Rad Laboratories) using a temperature program starting with 3 min at $95^{\circ} \mathrm{C}$ followed by 40 cycles consisting of $15 \mathrm{~s}$ at $95^{\circ} \mathrm{C}$ and $45 \mathrm{~s}$ at $57^{\circ} \mathrm{C}$. In addition, a fraction of the PCR mixture was analysed on a $1 \%$ agarose/ethidium bromide gel to check the size of the amplified DNA fragment. The RT-PCR primers were designed using (Beacon Designer 5.01 (Premier Biosoft International, Palo Alto, CA, USA) to obtain primers that have close to identical melting temperatures and do not form secondary structures with each other in the given PCR conditions. Primer sequences are listed in the chapter's Appendix. All reactions were performed on a combination of RNA samples, collected independently and pooled after RNA extraction.

\section{Functional classification criteria}

For the microarray analysis, the 20115 tomato probes were designed from sequences deposited in the tomato TGI database (Van der Hoeven et al., 2002). Thus, the annotations of the gene sets on the microarray were taken from the DFCI Tomato Gene Index (LGI), which integrates research data from all international tomato gene research projects. Where biological ontology was not clear, the UniProt Knowledgebase provided further functional information. All microarray expression data are available at GEO under the series entry GSE24805.

\section{Pollen germination}

Two replicates of three anther cones of freshly opened flowers were cut and 500uL of pollen germination media containing 20mM MES pH6.0, 3mM Ca(NO3)2, $1 \mathrm{mM} \mathrm{KCl,} 0.8 \mathrm{mM} \mathrm{MgSO} 4,1.6$ $\mathrm{mM}$ boric acid, 2.5\% (w/v) Sucrose and 24\% (w/v) PEG 4000 were added. After vortexing to release the pollen grains, incubation was done over night at room temperature, in a rotating shaker. Pollen germination was examined under a light microscope with the $10 \mathrm{X}$ objective. Scoring of the germination was performed in 5 frames. Mean germination percentages and SD were calculated. 


\section{Results and discussion}

In order to analyse the dynamics of transcriptional responses to moderate heat stress (MHS) in meiotic anthers of tolerant and sensitive genotypes, the temperature range for the experiment was chosen based on agronomically relevant temperatures shown to have a significant effect on pollen grain development, rather than using classical heat shock conditions of $42^{\circ}-45^{\circ} \mathrm{C}$. We focused our analysis of gene expression on whole meiotic anther cones isolated from flower buds of plants that were exposed to MHS $\left(32{ }^{\circ} \mathrm{C} / 26^{\circ} \mathrm{C}\right.$, day/night) for up to $30 \mathrm{~h}$, and compared this to standard temperatures $\left(26{ }^{\circ} \mathrm{C} / 18{ }^{\circ} \mathrm{C}\right.$ day/night). To analyse the heat stress response we used two genotypes previously characterized by plant breeders as relatively tolerant and sensitive. Figure 1 shows the phenotype of flowers, anthers and pollen viability of HS1 and FR grown under control conditions and two weeks of MHS. While the flower morphology and pollen germination remain largely unaffected by MHS in the HS1 genotype, in FR, MHS results in slightly smaller flowers with malformed anther cones and significantly reduced pollen germination.

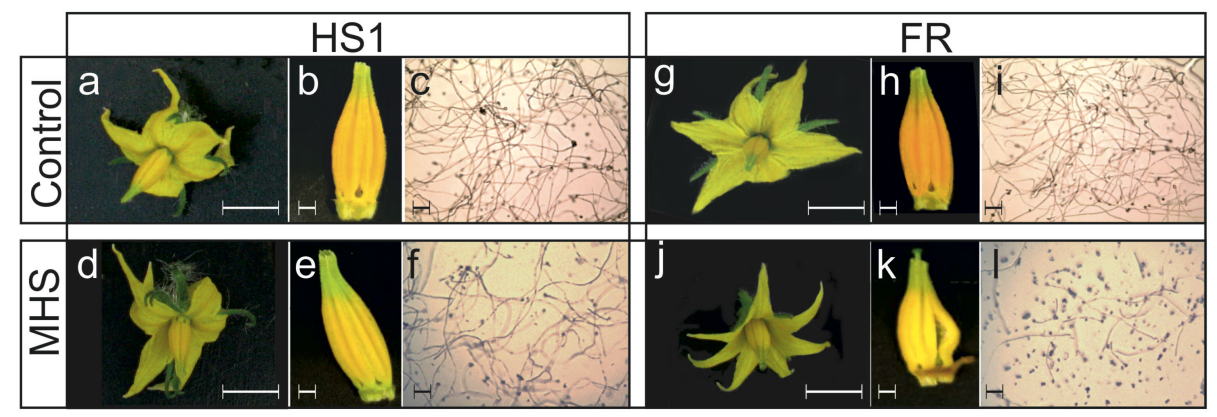

Figure 1. Tomato phenotypes under moderate heat stress. Comparison of flower and anther development under control and MHS conditions (two weeks) in the tolerant genotype HS1 and the sensitive FR. Panels a, d, g, j: whole flowers; panels b, e, h and k: isolated anther cones; panels c, f, i and l: germinating pollen. Size bars represent 10 and $3 \mathrm{~mm}$ respectively.

\section{Microarray analysis complements cDNA-AFLP analysis and indicates differential responses to MHS in heat-tolerant and heat-sensitive genotypes}

In tomato, microarray technology have been previously used to dissect stress responses and already enabled identification of candidate genes for tolerance to both biotic and abiotic stresses such as high temperature, low temperature, salt stress and pathogen attack (Frank et al., 2009; Gabriels et al., 2006; Zhang and Scheller, 2004; Zhu, 2001). We used the Combimatrix Tomato Array 1.0 to compare and analyse the response to heat in a tolerant and in a sensitive genotype. Modulation of gene expression in the two genotypes was analysed using multiclass, paired, and un-paired Significance Analysis of Microarray; SAM (Tusher et al., 2001). The microarray analysis indicates differential responses to heat stress in a heat-tolerant and a heat-sensitive tomato genotype. Since cDNA-AFLP demonstrated changes in gene expression in the MM tomato genotype early on during MHS, we chose an early time frame ( 0,2 and $6 \mathrm{~h})$ to compare the transcriptome of meiotic anthers exposed to MHS for two contrasting genotypes HS1 - a heat-tolerant hybrid that sets fruit at temperatures as high as $38^{\circ} \mathrm{C}$ in the field and FR - a relative heat-sensitive hybrid. Given the current global climate change projections (Solomon et al., 2009), plants that can trigger a suitable build-up of molecular mechanisms to prevent heat damage (Mittler et al., 2006) are very likely to become more important. The effects of environmental factors are often seen in gene expression changes and as predominantly downregulation of all gene programs for sensitive genotypes or in the case of tolerant genotypes as upregulation of protective mechanisms (Frank et al., 2009; Sun et al., 2010). 
To evaluate the relationship between genome-wide expression profiles of the two genotypes in relation to the heat treatment, SAM multiclass analysis was performed for three consecutive time points, 0h-2h-6h (Supplementary Materials, SAM multiclass). 56 genes in HS1 and 75 genes in FR significantly changed in expression over the entire duration of the treatment, for the false discovery rate (FDR) of $5 \%$. Transient up-regulation at the $2 \mathrm{~h}$ time point in both genotypes was the most common profile comparable to that of cDNA-AFLP experiment. Interestingly, up-regulation showed a marked genotypic difference with 12 genes up-regulated in HS1, in comparison to only 5 in FR. Down-regulation of gene expression was as expected, the second most frequently observed modulation for FR (16 genes) but not for HS1 (9 genes). These modulation types also show a good correlation with the cDNA-AFLP data, where the MM genotype is also characterised by a considerable down-regulation of gene expression. These results suggest that the difference in tolerance to MHS between the two genotypes is mainly associated with fewer and weaker transcriptomic responses in the heat-tolerant genotype.

A more in depth analysis of the heat response per genotype was obtained by looking at differentially expressed genes between three pair wise comparisons with a SAM paired analysis: $\mathrm{t} 0-\mathrm{t} 2$, t2-t6, and t0-t6 (Supplementary Materials, SAM paired). The statistical nature of the SAM paired analysis reveals supplementary information on subtle differences in the response to heat between the two genotypes, particularly in the sensitive genotype. We detected 74 genes in HS1 and 137 in FR, which exhibited significant changes in expression ( $\mathrm{FDR}=5 \%$ ). As in the multiclass analysis, the majority were significantly different for the $2 \mathrm{~h}$ time point ( $\mathrm{t} 0-\mathrm{t} 2)$ with 38 genes significantly changed in HS1 compared to 72 genes in FR. The SAM paired analysis also shows that, in the heat-tolerant HS1 genotype, less transcriptional changes are invested into reacting to the stress than in the heatsensitive FR. Overall, the paired analysis shows that HS1 up-regulates 56 and down-regulates 49 genes, while FR up-regulates 72 genes and down-regulates 109, clearly more when compared to the number of down-regulated genes in the heat-tolerant genotype. The SAM paired analysis supports and also provides more depth to the SAM multiclass, suggesting not only that the difference in tolerance between the two genotypes is indeed associated with a lower transcriptional response of the heattolerant genotype but also with more functional classes and members affected in the sensitive genotype.

To detect if significant differences also can be found between the two genotypes prior to the heat treatment, we used the unpaired SAM analysis (Supplementary Materials, SAM unpaired). 24 genes were significantly different between HS1 and FR (FDR=5\%). Of these, 4 are significantly higher in the heat-tolerant genotype, while the other 20 genes are at significantly higher levels in the heat-sensitive genotype. A previous study on salt cress proposed that the salt tolerant plant had a smaller number of salt-regulated genes because of the constitutive and active over-expression of stress-related genes even under standard growth conditions (Taji et al., 2004). Similarly, Frank et al. (Frank et al., 2009) showed that constitutive expression of a heat shock transcription factor and several heat shock proteins mark these genes as candidates for taking part in microspore thermotolerance. We suggest that in HS1, the differentially expressed genes prior to heat treatment may represent a constitutive tolerance to MHS.

In summary, the statistical analysis of gene expression during MHS reveals a differential response to heat in the two genotypes, in terms of number of significantly differentially expressed transcripts, their levels of expression and the functional classes they belong to. Moreover, the analysis also shows that significant differences in gene expression are present prior to the commencement of the MHS treatment. 


\section{Main components determining gene expression modulation under MHS}

To identify the main components determining the changes in gene expression in response to MHS, an O2PLS multivariate regression analysis of the data set (Bylesjo et al., 2007) was done, using the set of 95 significantly differentially expressed genes obtained by multiclass SAM analysis. The two variables chosen were: as X the 95 transcripts, and as $\mathrm{Y}$, the 6 comparison classes (HS0, F0, HS2, F2, HS6 and F6). The OPLS shows four major components of variation (Figure 2). Component 1 in T1/T2 plot separates the three time points distinctly (Figure 2A); in particular T1 separates time 0 from 2 and 6. This result confirms that there is a rapid gene expression response to MHS. In addition, the O2PLS analysis shows a good clustering of the biological repeats in both genotypes. Component 3 in the T1/T3 plot (Figure 2B) separates the HS1 and FR genotypes at $0 \mathrm{~h}$ time point and also, to a lesser degree at $2 \mathrm{~h}$ time point. This indicates that prior to the MHS treatment there is a clear difference in gene expression in the meiotic anthers of these two genotypes. Component 4 in the T1/T4 plot indicates a genotypic difference in the response to the increased period of treatment (Figure 2C). We conclude from this analysis, that the major component of variation in transcriptional changes is based on the innate genotypic differences between HS1 and FR prior to the heat stress application.

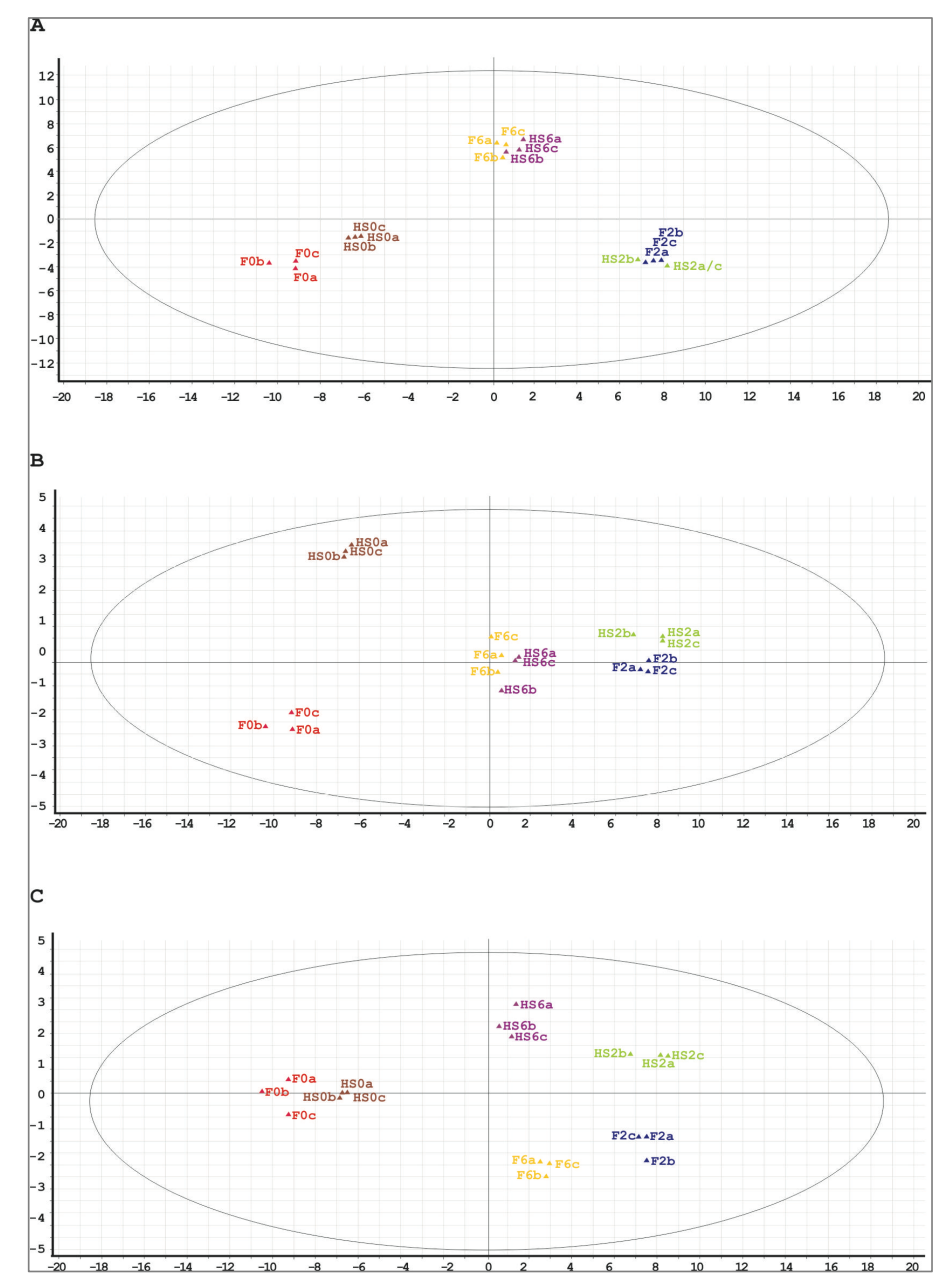

Figure 2. O2PLS analysis of components of variation in gene expression in the microarray experiment. The main components of the changes in gene expression in response to MHS were determined using an O2PLS analysis with SIMCA P+. The plots of predictive component (T2, T3 and T4) versus orthogonal component 1 (T1) are presented (A, B \& C). HS1-0a, b and c, HS1-2a, b and c, HS1-6a, b and c, FR-0a, b and c, , FR-2a, b and $\mathrm{c}$ and FR-6a, b and c represent the three biological replicates ( $\mathrm{a}, \mathrm{b}$ and $\mathrm{c}$ ) for each experimental time-point ( 0,2 and $6 \mathrm{~h}$ ) for each genotype ( HS1 and FR). Each component clearly discriminates between the two genotypes. 


\section{Transcript profile clustering under MHS}

To analyse the gene expression profiles for each genotype, a Hierarchical Clustering (HCL) analysis was applied to the same set of 95 significantly differentially expressed genes used in the O2PLS (Figure 2). The wide transcriptional response in the heat-sensitive genotype (FR) also becomes clear with the difference between the numbers in both data sets. The largest cluster for both genotypes corresponds to a transient induction of gene expression activity, whereas up-regulation and downregulation are the second most occurring pattern in HS1 and FR respectively. The common probes in the different genotypes are indicated with connecting lines in Figure 3. This set has a largely similar expression profile between the two genotypes. 20 genes showing significant changes over the three time points are unique to the HS1 genotype whereas there are 39 unique differentials in the FR genotype (Figure 3). We conclude that HS1 shows a less extensive response in terms both of the intensity of transcriptional changes and the number of genes that are induced during MHS.

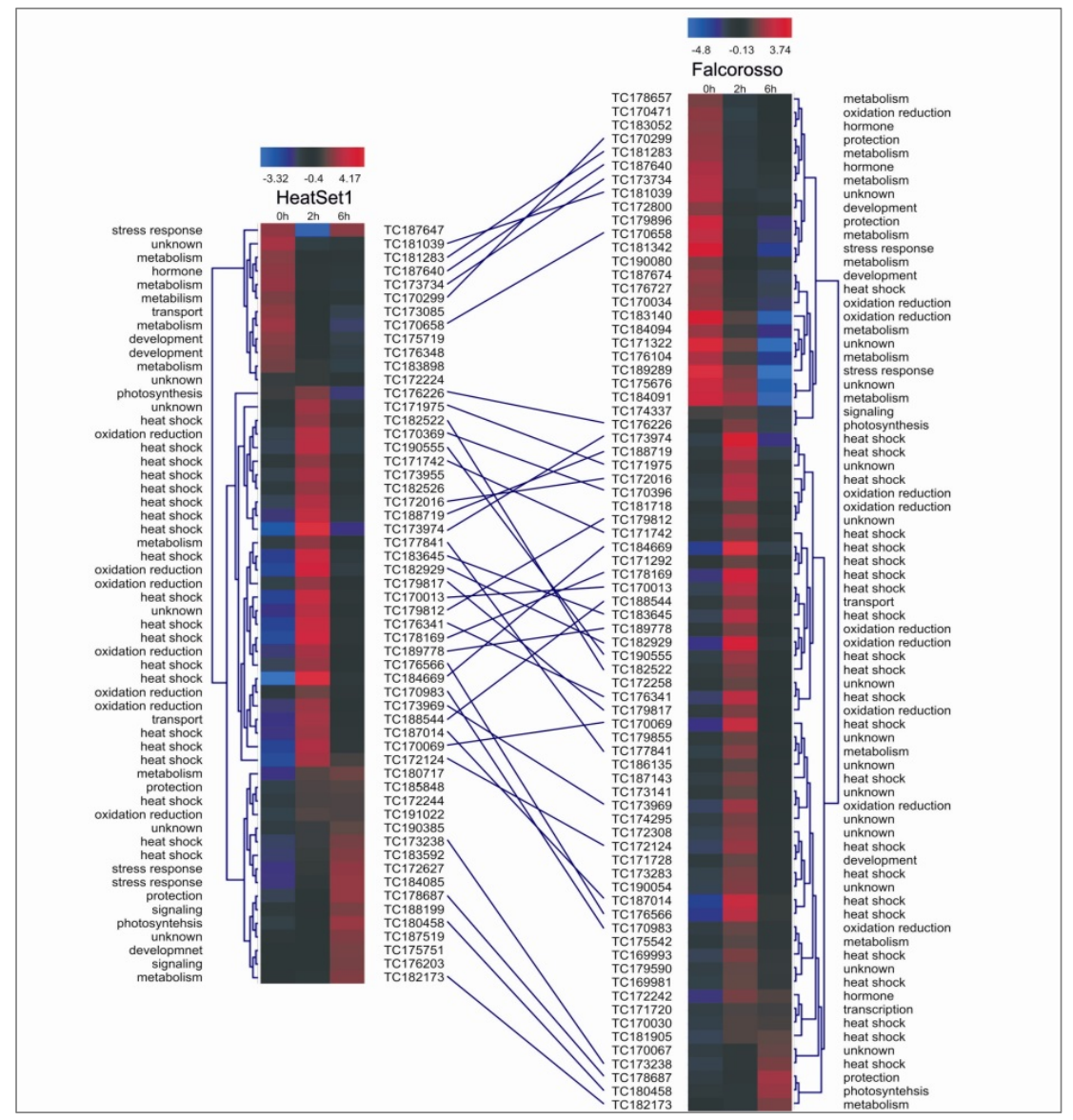

Figure 3. Euclidian clustering of significantly differentially expressed genes in the heat-tolerant genotype (HS1) and the heat-sensitive genotype (FR). Heat maps of gene expression are shown with high expression shown in shades of red and low expression shown in blue. A dendogram of the expression profiles is shown to the left in HS1 (Heat Set 1) and the right in FR (Falcorosso). On either side of the heat maps, the TC number of the relevant probe is shown and the functional classification of the relevant gene. TCs common to both genotypes are indicated with lines linking the heat maps.

\section{Transcript profile abundance}

A Self Ordering Map (SOM) analysis was created to obtain an overview of the predominant expression profiles of significantly changed genes as defined by the SAM multiclass. Six clusters were generated in the SOM comprising 27, 3, 4, 21, 16, and 24 genes respectively (Clusters 1-6 
respectively; Figure 4). The analysis shows that the predominant expression profile resulting from MHS is a transient up- regulation of gene expression (Cluster 3, 4 and 5). The next most abundant pattern was down- regulation (Cluster 1 and 2). Other patterns were found less frequently (Cluster 6). These expression profiles did not differ strongly over time in the two genotypes. We conclude that HS1 gene expression is not so strongly affected by MHS compared to FR and that most expression changes involve a transient induction in both genotypes. Furthermore, prior to MHS, the heat-tolerant HS1 differs in constitutive gene expression from the heat-sensitive FR.

The results from the SOM analysis show that the predominant modulation pattern in the two genotypes is transient induction, followed by an up-regulation pattern in the heat-tolerant and downregulation pattern in the heat-sensitive genotype. Here again, the gene identity and modulation intensity for each genotype suggest that the difference in tolerance to MHS is associated with fewer and weaker transcriptomic responses in the heat-tolerant genotype.

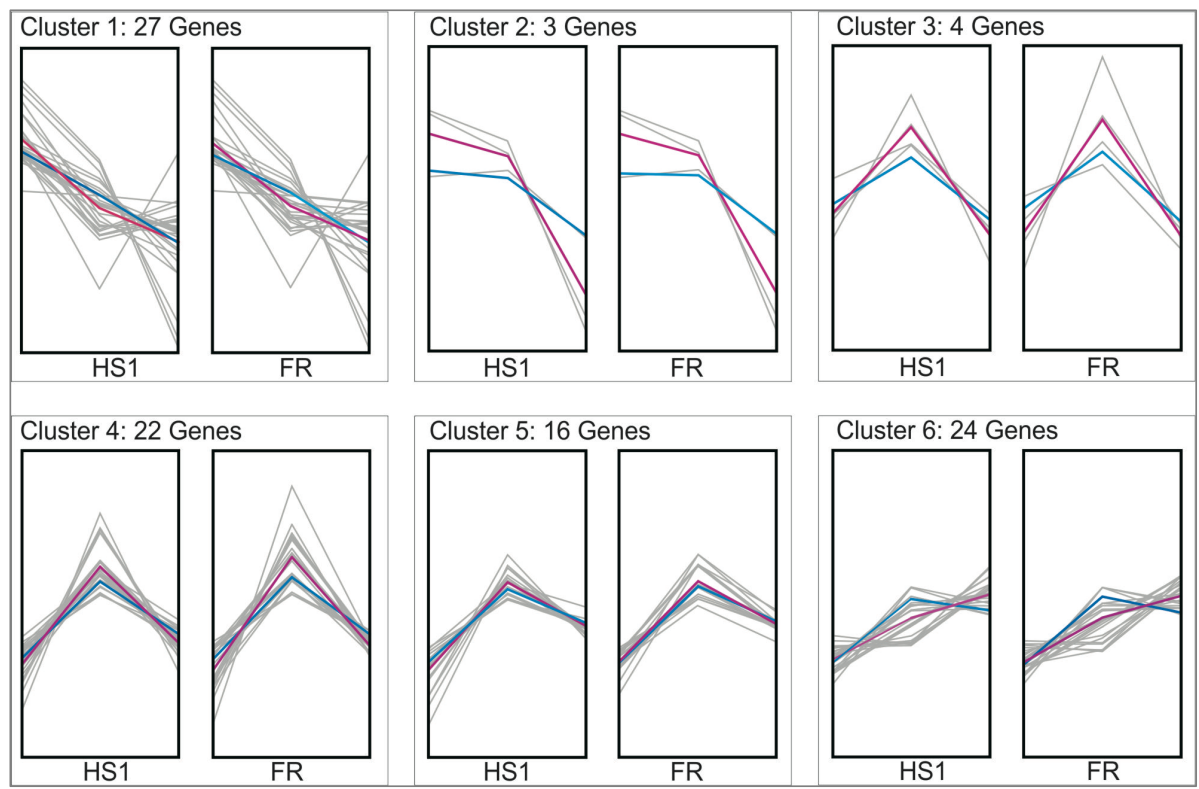

Figure 4 Self Ordering Map analysis (SOM) of expression profiles from the microarray experiment. 6 clusters of SOMs are depicted, with FR indicating the heat-sensitive and HS1 the heat-tolerant genotype.

\section{Functional groups modulated by MHS}

The functional classification derived from DFCI gene index and UniProt annotation, illustrates the processes primarily affected in each of the genotypes. Genes were organized according to the metabolic processes in which they appear to be involved. Figure 5 shows the number of genes of each functional set illustrated as separate pie charts for the two genotypes and statistical analyses (SAM paired and multiclass). The multiclass SAM analysis of the stress response (upper section of Figure 5) reveals that the heat-tolerant and heat-sensitive genotypes react similarly, involving analogous functional groups: protection and repair, signalling and transcription, metabolism and development. Of the genes identified, the majority belongs to the protection and repair group (heat shock proteins, oxido-reductive molecules, stress response and protection) in both genotypes, while the metabolism and development group is much more highly represented in the heat-sensitive genotype and shows involvement of additional response pathways such as carbohydrate metabolism and hormone-related genes, according to the SAM paired analysis (lower part of Figure 5). By analogy, a transcriptomic analysis of tolerant and sensitive wheat strains indicates transcripts coding for heat shock proteins, heat shock and other transcription factors are already turned on during acclimation for $3 \mathrm{~h}$ at $34^{\circ}$ (Qin et al., 2008). 


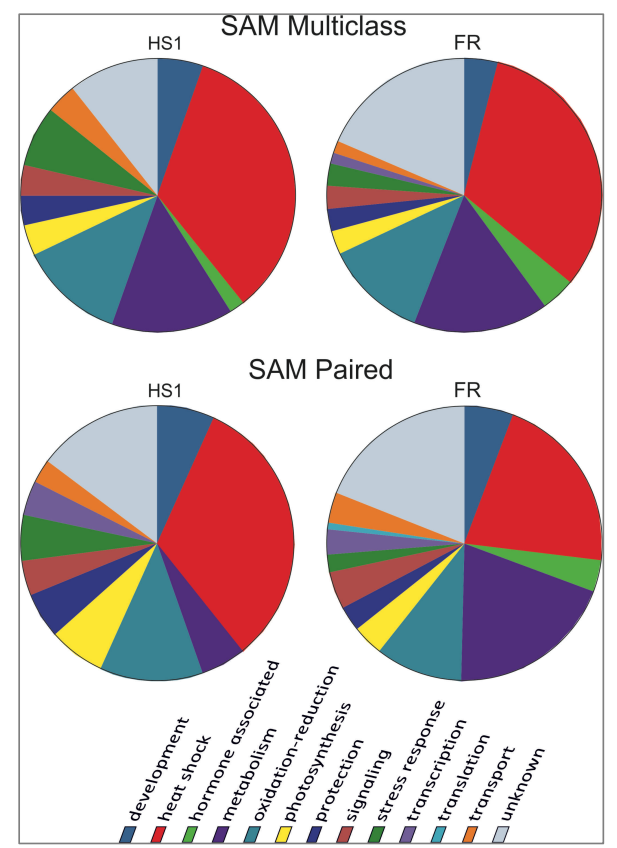

Figure 5. Pie charts of the probable functional categorisation of the transcripts found to be significantly differentially expressed in the microarray experiment. The two charts reflect the general (SAM multiclass) and in depth (SAM Paired) moderate temperature stress response in HS1, the heat-tolerant, and FR, the heat-sensitive genotype. The functional classes are colour coded

\section{Protection and repair transcripts represent the majority of genes affected by MHS}

From the SAM paired analysis, the highest induced transcripts during the first $2 \mathrm{~h}$ of MHS in the tolerant genotype belong to the protection and repair genes and are similar to: AthHsp22.3 (TC185802, fold induction 1500), a class I heat shock protein (TC184669, fold induction 170), a class III sHsp (TC173974, fold induction 106), the cytosolic ascorbate peroxidase (TC182989, fold induction 36.6) and a class I sHsp (TC178169, fold induction 35.8). The highest induced transcripts in the sensitive genotype during the first two hours of MHS are a class I heat shock protein (TC184669, fold induction 73), a DNA-J (176514, fold induction 55), a cytosolic ascorbate peroxidase (TC182929, fold induction 34) and mitochondrial sHsp (TC187014, fold induction 32).

However, the highest induction over the entire duration of MHS was recorded in both genotypes and consists of a similar group in each genotype: class I sHsp 17.6 (TC178169), class II sHsp 17.6 (TC170069), and mitochondrial sHsp (TC187014). Of these, only the class I sHsp 17.6 is higher induced in the tolerant genotype (fold induction 36 as compared to 26 in FR). A single transcript corresponding to a DNA-J like protein (TC176727) was down-regulated in FR over the 6h of MHS, but during the last $4 \mathrm{~h}$ of MHS, 15 heat shock proteins were down-regulated in expression in FR and 18 in HS1.

The heat-tolerant genotype not only reacted differently from the heat-sensitive during MHS in terms of gene number, but also exhibited a characteristic gene expression pattern, characterised by very high expression levels of protection and repair genes. The heat shock Hsp82 (TC170030) and the gene coding for mitochondrial small heat shock protein (msHsp) LeMtHSP (TC187014) are highly expressed in HS1 at time point 0h prior to the MHS. Another set of genes are responsive only in the heat-sensitive genotype, among which the heat shock proteins Hsa32 (TC191544), Rof1 (TC187143) and ROC7 (TC175253) genes required for protein folding. The extent to which the oxido-reduction processes are affected by MHS in the heat-sensitive genotype becomes evident from the differential regulation of genes such as the carotenoid cleavage dioxygenase 1 (TC181718), a steroid alphareductase, (TC170471), a flavonol synthase (TC172800) and a flavoprotein (TC83140. The stress 
response of the heat-sensitive genotype can be seen from repression of transcripts similar to pepsin A (TC171322) and subtilisine (TC181342). Unlike the heat-sensitive genotype, the heat-tolerant genotype HS1 shows fewer genes significantly induced in the protection and repair group.

In conclusion, the heat-tolerant genotype HS1 reacts to MHS by enhancing the expression of heat shock proteins, oxido-reductive, transport and stress protective transcripts. In stark contrast, the heat-sensitive FR shows a more extensive, wider transcriptomic response characterised first by a complementary modulation pattern as compared to the heat-tolerant genotype; e.g. transport and signalling genes are down-regulated in the heat-sensitive and up-regulated in the heat-tolerant. Second, the stress response in the heat-sensitive genotype is characterised by an increased expression of a more diverse range of functional classes including transcription, photosynthesis, hormone related and protection genes which are increased, while transport and carbohydrate metabolism are reduced in expression. Third, although the two genotypes involve similar functional classes in the response to heat, each genotype modulates "common" and "specific" genes, as described earlier. The genotypespecific modulated transcripts are of particular interest for the present work as their annotation may provide clues to the putative mechanism of heat tolerance.

The results obtained in the present study are similar to those obtained by Frank et al. (Frank et al., 2009), where the heat-tolerant genotype is shown to modulate fewer genes in response to heat, but also similar to results on other abiotic stresses (Page et al., 2010; Swindell et al., 2007; Zhou et al., 2007). The most marked differences between the two genotypes can be seen in the heat shock, metabolism and oxido-reduction groups: $32 \%$ (HS1) respectively $20 \%(\mathrm{FR})$ of the modulated genes belong to the heat shock group, $12 \%$ (HS1) respectively $20 \%$ (FR) belong to metabolic genes group and $8 \%$ (HS1) respectively $10 \%$ (FR) belong to the oxido-reduction group. It appears that the heattolerant genotype modulates mainly genes in the heat shock group while the heat-sensitive genotype modulates genes of the heat shock and metabolic groups in equal fractions, processes which may underpin the better performance of the heat-tolerant genotype under elevated temperatures.

\section{Metabolism and development transcripts are the second most affected by MHS}

Interestingly, in comparison to the heat-tolerant genotype, all development-related genes significantly changed in expression by MHS were very highly expressed in the heat-sensitive genotype FR. In the metabolism group it is interesting to note that FR exhibits increased expression levels of carbohydrate metabolism genes as well, for example a transcript coding for beta-amylase (TC178310) is induced 3 fold in FR during the first $2 \mathrm{~h}$ of MHS but then it is repressed again._ $\beta$-amylase induction and the resultant maltose accumulation may function as a compatible-solute stabilizing factor in the chloroplast stroma in response to acute temperature stress (Kaplan and Guy, 2004).

\section{Transcription and signalling transcripts are also affected by MHS}

Unlike the heat-tolerant genotype HS1, the heat-sensitive FR shows a different genetic make-up with regard to transcription, translation and signalling. For example, transcripts similar to the ARF5 (TC182088) and a 26S ribosomal protein (TC177412) are expressed at higher levels in the heatsensitive genotype, which implies an involvement of auxin and de novo protein synthesis in FR, under MHS. The ethylene-responsive transcriptional co-activator (TC171720) is also affected (transiently induced at $2 \mathrm{~h}$ ) by heat only in the sensitive genotype, which may also reflect the sensitivity of FR to MHS. It is known that the A. thaliana ortholog, AtMBF1c, enhances tolerance to heat and osmotic stress when over-expressed in $A$. thaliana (Suzuki et al., 2008). Unlike the heat-tolerant genotype HS1, FR reacts with a different set of signalling partners and transcriptional regulators, downregulating after the $6 \mathrm{~h}$ of MHS a photoperiod responsive protein (TC177921) and an ARF5-like 
transcript (TC182088). In Arabidopsis hypocotyls, high temperature (here $29^{\circ} \mathrm{C}$ ) causes an increase in free IAA, and this mediates a marked cell elongation response. Therefore temperature signals may modify auxin synthesis or distribution in the plant, and this could represent a growth-regulating mechanism (Gray et al., 1998). The heat-tolerant genotype reacts to heat by continuously increasing the expression of an Adagio 3-like transcript (TC188199) while another Bell transcriptional regulator (TC175335) is decreased at $6 \mathrm{~h}$ of MHS.

\section{MHS differentially affects anther development in tolerant and sensitive tomato genotypes}

In order to confirm the observed microarray expression profiles, q-PCR experiments were carried out. The expression pattern of several candidate transcripts from different functional classes was confirmed in the heat tolerant HS1 and the heat-sensitive FR genotypes, showing that heat has a clear influence on gene expression ( figure 6).

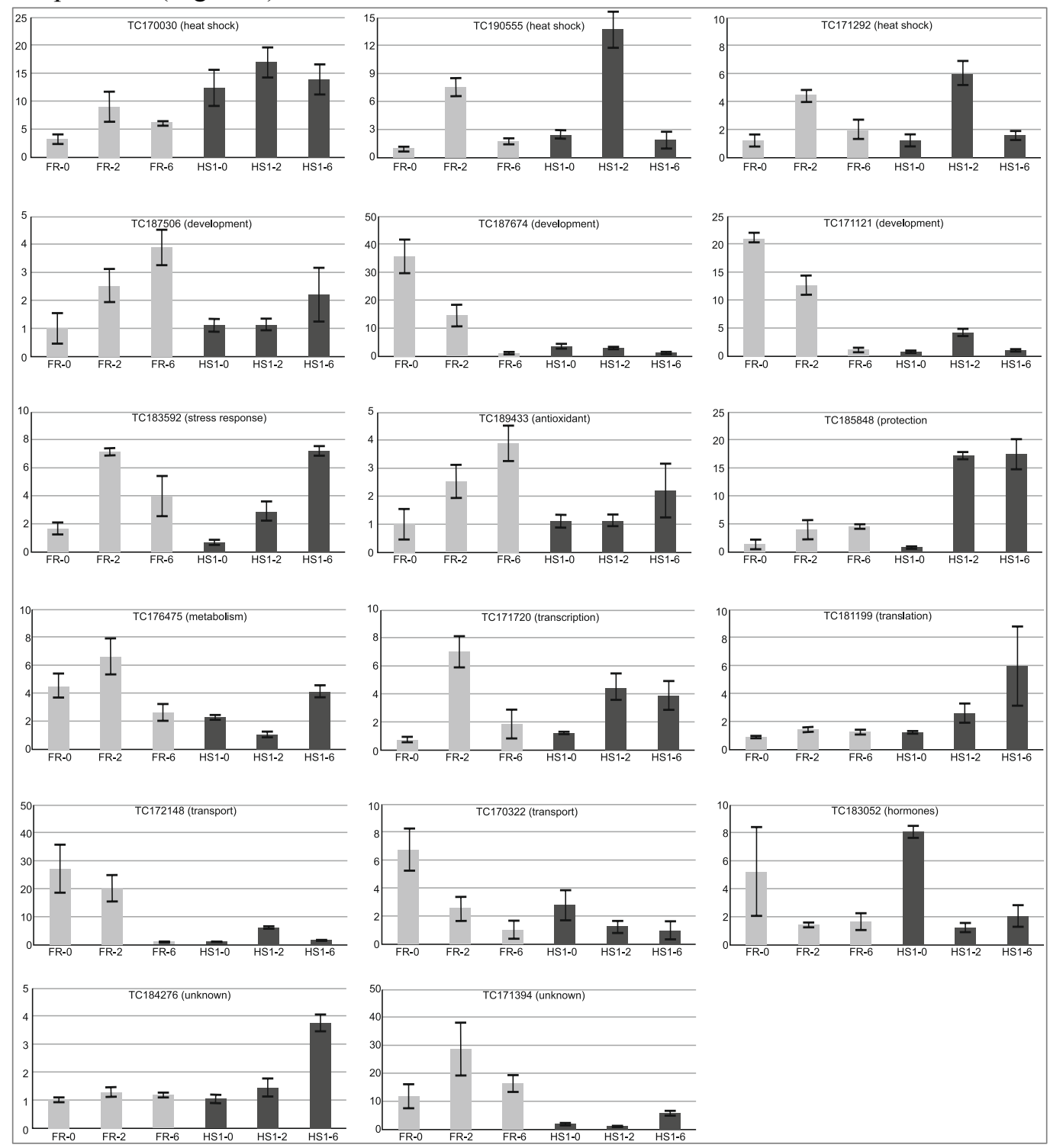

Figure 6. Validation of the microarray profiling with RT-PCR. Candidate genes in FR and HS1. Individual candidate genes are shown as TC numbers with the processes in which they are involved in parenthesis. RTPCR was done using RNA isolated from pools of meiotic anther cones of the sensitive (FR; light grey bars) and tolerant (HS; dark grey bars) genotypes at 0h (FR-0, HS-0), 2h (FR-2, HS-2) and 6h (FR-6, HS-6) after initiation of the MHS treatment. The fold induction is given on the y-axis. The error bars are given as standard deviation. 
Additional confirmation and correlation was obtained by performing q-PCR in another pair of contrasting genotypes: Saladette (heat-tolerant) and Pull (heat-sensitive). Transcripts coding for heat shock, transport and protection proteins show similar expression patterns to the ones observed from the SAM analyses. In addition, the second pair of contrasting genotypes showed a similar modulation in gene expression (Figure 7).

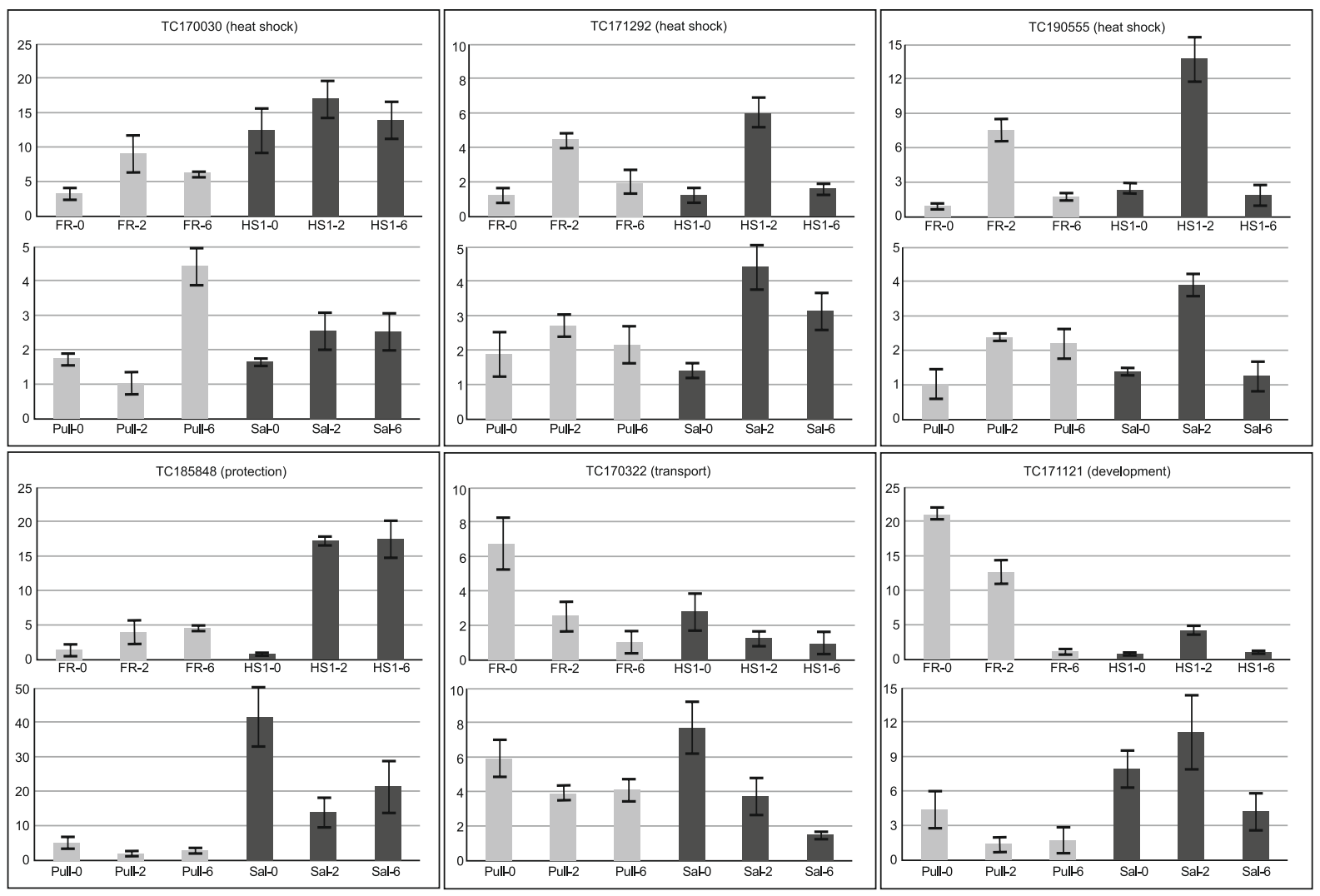

Figure 7. Validation of the microarray profiling with RT-PCR. Six candidate genes in heat-sensitive and heattolerant genotypes, FR, Pull, HS1, Saladette. Individual candidate genes are shown as TC numbers with the processes in which they are involved. RT-PCR was done using RNA isolated from pools of meiotic anther cones of the sensitive (FR, Pull; light grey bars) and tolerant (HS1, Sal; dark grey bars) genotypes at 0h (FR-0, HS-0, Pull-0), 2h (FR-2, HS-2. Pull-2) and 6h (FR-6, HS-6. Pull-6) after initiation of the MHS treatment. The fold induction is given on the $\mathrm{y}$-axis. The error bars are given as standard deviation.

\section{Conclusions}

Our transcript profiling experiments and those of others (Frank et al., 2009) demonstrate that many genes, involved in a wide variety of processes, are modulated by moderate heat stress. Many of these genes show a differential expression both in sensitive and in relatively tolerant genotypes that differ only in amplitude of induction. This suggests that tolerance is based on fine-tuning and quantitative expression of a large number of genes. It is difficult to say whether the differences observed in our experiments are the basis of heat tolerance or whether the changes result directly in a better performance of the plant under stress conditions. Comparisons of the temporal gene expression patterns indicate possible different mechanisms of high temperature tolerance in tomatoes. A significant set of differentially expressed genes show differences prior to the onset of the MHS. This may be an indication that these genes predispose the tolerant genotype to be more resistant. Furthermore, the large transcriptomic response in the sensitive cultivar compared to the tolerant supports this model. A selection of tomato during breeding and cultivation is likely to be based on a small set of domestication genes causing little genetic variation in the tomato germplasm (Paran and 
van der Knaap, 2007). Such allelic variation may have its basis in SNPs in promoter regions leading to the observed expression changes. 


\section{Chapter Appendix}

Table 1. Primers used for RT-PCR validation:

\begin{tabular}{|c|c|}
\hline TC189433F & 5'ACTTTGATCGTACAGTAGTTGAC \\
\hline TC189433R & 5'-TGCTTCCCTCATTGTTTCATTAC \\
\hline TC187674F & 5’-TGATCCATCTTAGGTAATTTAGG \\
\hline TC187674R & 5'-CGTTATACTAGAACATAAACTTGG \\
\hline TC187586F & 5'-TGACCCACCATATTGTGAACTAAG \\
\hline TC187586R & 5`-GCACCCAATAGAACAAATGTAACG \\
\hline TC187506F & 5’ATAACTCAACTAGTATGTGGATGG \\
\hline TC187506 R & 5`-GTTCGTTAACTTTTCTATTAGGAGAG \\
\hline TC183052F & 5`-TACTTAAACAACTTCCGTCTCTCG \\
\hline TC183052 R & 5`- AGCCACTCAAGTGAATAATGGCGT \\
\hline TC171121F & 5'-ATCAAACCAAACTCACTTGTTAG \\
\hline TC171121R & 5`-TGGCAATATACCTTGTTAGGAG \\
\hline TC176475F & 5'-CTGTTCCCGCTGTGGTGTTCC \\
\hline TC176475 R & 5`- CCTCGCTCAAGTTAGAAGCTCC \\
\hline TC183592F & 5’-TGGCCTGAACTTGTTGGGAAATC \\
\hline TC183592R & 5'-TATTTATGCTGAAATAGTAGAGTACAT \\
\hline TC181199F & 5`-CTTTCGTTTGCTTAGCCAGTACC \\
\hline TC181199 R & 5'-TGTCTGATATGAGTTCTCCAGTCTC \\
\hline TC172148F & 5'-GGGACTAATAGAGTTCAACCTTG \\
\hline TC172148R & 5’-ATCACAACTGCTATGAGAATGG \\
\hline TC171720F & 5'-CAGAAGCCAAAGATGAAGG \\
\hline TC171720R & 5’-ACAACCTGCGTCCTTTC \\
\hline TC171394F & 5'-AGCCGTCTTGTTTTATACTATGC \\
\hline TC171394R & 5'- TTAACTTGTAAGAATGAGGTCTCC \\
\hline TC184276F & 5'-GGTGTAAGGCCGGTTATTGGATTC \\
\hline TC184276R & 5'-ССТССТСССТCСАTTGCTAGACC \\
\hline TC $170030 \mathrm{~F}$ & 5'-AACAAGATTCTCAAGGTGATTAGG \\
\hline TC 170030R & 5'-GGATTATGTTACTAGGATGAAGGAG \\
\hline TC $170322 \mathrm{~F}$ & 5'-GTTAGAGTTGGTTGGATGTTTGG \\
\hline TC 170322R & 5'-GGCTATTGTCCATTTAACTTTCCC \\
\hline TC $171292 \mathrm{~F}$ & $5^{\prime}$-AGGACGGTGATGAGGAAATGAAGAAG \\
\hline TC 171292R & 5'-CAGTATGTTGCTTGAATGGTCGTT \\
\hline TC $190555 \mathrm{~F}$ & 5'-CCTATGGTCGTGATCTTGTG \\
\hline TC 190555R & 5'-GTTAGGCTTATAGCATTGGATATG \\
\hline TC $185848 \mathrm{~F}$ & 5'-AGCAAGAAGTACCATGTATCC \\
\hline TC 185848R & 5'-CCTTATGAGTAACCTGTTGTTG \\
\hline
\end{tabular}




\section{References}

Aarts, M.G., Hodge, R., Kalantidis, K., Florack, D., Wilson, Z.A., Mulligan, B.J., Stiekema, W.J., Scott, R. and Pereira, A. (1997) The Arabidopsis MALE STERILITY 2 protein shares similarity with reductases in elongation/condensation complexes. Plant J. 12, 615-623.

Abiko, M., Akibayashi, K., Sakata, T., Kimura, M., Kihara, M., Itoh, K., Asamizu, E., Sato, S., Takahashi, H. and Higashitani, A. (2005) High-temperature induction of male sterility during barley (Hordeum vulgare L.) anther development is mediated by transcriptional inhibition. Sexual Plant Reproduction 18, 91-100.

Ariizumi, T., Hatakeyama, K., Hinata, K., Sato, S., Kato, T., Tabata, S. and Toriyama, K. (2003) A novel male-sterile mutant of Arabidopsis thaliana, faceless pollen-1, produces pollen with a smooth surface and an acetolysis-sensitive exine. Plant Mol. Biol. 53, 107-116.

Brukhin, V., Hernould, M., Gonzalez, N., Chevalier, C. and Mouras, A. (2003) Flower development schedule in tomato Lycopersicon esculentum cv. sweet cherry. Sexual Plant Reproduction 15, 311-320.

Bylesjo, M., Eriksson, D., Kusano, M., Moritz, T. and Trygg, J. (2007) Data integration in plant biology: the O2PLS method for combined modeling of transcript and metabolite data. Plant $J$. 52, 1181-1191.

Chen, Y.C. and McCormick, S. (1996) sidecar pollen, an Arabidopsis thaliana male gametophytic mutant with aberrant cell divisions during pollen development. Development 122, 3243-3253.

Cnudde, F., Hedatale, V., de Jong, H., Pierson, E.S., Rainey, D.Y., Zabeau, M., Weterings, K., Gerats, T. and Peters, J.L. (2006) Changes in gene expression during male meiosis in Petunia hybrida. Chromosome Res 14, 919-932.

Drews, G.N. and Goldberg, R.B. (1989) Genetic control of flower development. Trends Genet. 5, 256261.

Frank, G., Pressman, E., Ophir, R., Althan, L., Shaked, R., Freedman, M., Shen, S. and Firon, N. (2009) Transcriptional profiling of maturing tomato (Solanum lycopersicum L.) microspores reveals the involvement of heat shock proteins, ROS scavengers, hormones, and sugars in the heat stress response. Journal of experimental botany 60, 3891-3908.

Gabriels, S.H., Takken, F.L., Vossen, J.H., de Jong, C.F., Liu, Q., Turk, S.C., Wachowski, L.K., Peters, J., Witsenboer, H.M., de Wit, P.J. and Joosten, M.H. (2006) CDNA-AFLP combined with functional analysis reveals novel genes involved in the hypersensitive response. $\mathrm{Mol}$ Plant Microbe Interact 19, 567-576.

Gibson, G. (2003) Microarray analysis: genome-scale hypothesis scanning. PLoS Biol 1, E15.

Goldberg, R.B. (1988) Plants: novel developmental processes. Science 240, 1460-1467.

Gray, W.M., Ostin, A., Sandberg, G., Romano, C.P. and Estelle, M. (1998) High temperature promotes auxin-mediated hypocotyl elongation in Arabidopsis. Proc Natl Acad Sci U S A 95, 7197-7202.

Hulskamp, M., Parekh, N.S., Grini, P., Schneitz, K., Zimmermann, I., Lolle, S.J. and Pruitt, R.E. (1997) The STUD gene is required for male-specific cytokinesis after telophase II of meiosis in Arabidopsis thaliana. Dev Biol 187, 114-124.

Kakani, V.G., Reddy, K.R., Koti, S., Wallace, T.P., Prasad, P.V., Reddy, V.R. and Zhao, D. (2005) Differences in in vitro pollen germination and pollen tube growth of cotton cultivars in response to high temperature. Ann Bot 96, 59-67.

Kamel, A., Qian, Y., Kolbe, E. and Stafford, C. (2010) Development and validation of a multiresidue method for the determination of neonicotinoid and macrocyclic lactone pesticide residues in milk, fruits, and vegetables by ultra-performance liquid chromatography/MS/MS. J AOAC Int 93, 389-399.

Kaplan, F. and Guy, C.L. (2004) beta-Amylase induction and the protective role of maltose during temperature shock. Plant Physiol 135, 1674-1684.

Kaul, M.L.H. (1988) Male sterility in higher plants. Berline:Springer-Verlag.

Kim, J.C., Lee, S.H., Cheong, Y.H., Yoo, C.M., Lee, S.I., Chun, H.J., Yun, D.J., Hong, J.C., Lee, S.Y., Lim, C.O. and Cho, M.J. (2001) A novel cold-inducible zinc finger protein from soybean, SCOF-1, enhances cold tolerance in transgenic plants. The Plant journal : for cell and molecular biology 25, 247-259. 
Levy, A., Rabinowitch, H.D. and Kedar, N. (1978) Morphological and physiological characters affecting flower drop and fruit set of tomatoes at high temperatures Euphytica 27, 211-218.

Levy, C., Donnadieu, M. and Halpern, G.M. (1977) [Demonstration of specific serum IgG with an "in vitro" radioimmunoassay (RAST IgG) (author's transl)]. Pathol Biol (Paris) 25, 505-508.

Lindquist, S. and Craig, E.A. (1988) The heat-shock proteins. Annu Rev Genet 22, 631-677.

Mittler, R., Kim, Y., Song, L., Coutu, J., Coutu, A., Ciftci-Yilmaz, S., Lee, H., Stevenson, B. and Zhu, J.K. (2006) Gain- and loss-of-function mutations in Zat10 enhance the tolerance of plants to abiotic stress. FEBS letters 580, 6537-6542.

Page, D., Gouble, B., Valot, B., Bouchet, J.P., Callot, C., Kretzschmar, A., Causse, M., Renard, C.M. and Faurobert, M. (2010) Protective proteins are differentially expressed in tomato genotypes differing for their tolerance to low-temperature storage. Planta 232, 483-500.

Paran, I. and van der Knaap, E. (2007) Genetic and molecular regulation of fruit and plant domestication traits in tomato and pepper. J. Exp. Bot. 58, 3841-3852.

Porch, T.G. and Jahn, M. (2001) Effects of high-temperature stress on microsporogenesis in heatsensitive and heat-tolerant genotypes of Phaseolus vulgaris. Plant Cell and Environment 24, 723-731.

Pressman, E., Peet, M.M. and Pharr, D.M. (2002) The effect of heat stress on tomato pollen characteristics is associated with changes in carbohydrate concentration in the developing anthers. Ann Bot 90, 631-636.

Qin, D., Wu, H., Peng, H., Yao, Y., Ni, Z., Li, Z., Zhou, C. and Sun, Q. (2008) Heat stress-responsive transcriptome analysis in heat susceptible and tolerant wheat (Triticum aestivum L.) by using Wheat Genome Array. BMC genomics 9, 432.

Sato, S. and Peet, M.M. (2005) Effects of moderately elivated temperature stress on the timing of pollen release and its germination in Tomato (Lycopersicon exculentum Mill.). J. Hort. Sci. Biotech. 80, 23-28.

Sato, S., Peet, M.M. and Gardner, R.G. (2004) Altered flower retention and development patterns in 9 tomato cultivars under elevated temperature. Hort. Sci. 101, 95-101.

Sato, S., Peet, M.M. and Thomas, J.F. (2002a) Determining critical pre- and post-anthesis periods and physiological processes in Lycopersicon esculentum Mill. exposed to moderately elevated temperatures. J Exp Bot 53, 1187-1195.

Sato, Y., Aoki, Y. and Matsuoka, M. (2002b) A loss-of-function mutation in the rice KNOX type homeobox gene, OSH3. Plant Cell Physiol 43, 44-51.

Sato, Y., Murakami, T., Funatsuki, H., Matsuba, S., Saruyama, H. and Tanida, M. (2000) Heat shockmediated APX gene expression and protection against chilling injury in rice seedlings. Journal of experimental botany 52, 145-151.

Solomon, S., Plattner, G.K., Knutti, R. and Friedlingstein, P. (2009) Irreversible climate change due to carbon dioxide emissions. Proc Natl Acad Sci U S A 106, 1704-1709.

Sun, W., Xu, X., Zhu, H., Liu, A., Liu, L., Li, J. and Hua, X. (2010) Comparative transcriptomic profiling of a salt-tolerant wild tomato species and a salt-sensitive tomato cultivar. Plant Cell Physiol 51, 997-1006.

Suzuki, N., Bajad, S., Shuman, J., Shulaev, V. and Mittler, R. (2008) The transcriptional co-activator MBF1c is a key regulator of thermotolerance in Arabidopsis thaliana. $J$ Biol Chem 283, 92699275.

Swindell, W.R., Huebner, M. and Weber, A.P. (2007) Transcriptional profiling of Arabidopsis heat shock proteins and transcription factors reveals extensive overlap between heat and non-heat stress response pathways. BMC genomics 8, 125.

Taji, T., Seki, M., Satou, M., Sakurai, T., Kobayashi, M., Ishiyama, K., Narusaka, Y., Narusaka, M., Zhu, J.K. and Shinozaki, K. (2004) Comparative genomics in salt tolerance between Arabidopsis and aRabidopsis-related halophyte salt cress using Arabidopsis microarray. Plant Physiol 135, 1697-1709.

Taylor, P.E., Glover, J.A., Lavithis, M., Craig, S., Singh, M.B., Knox, R.B., Dennis, E.S. and Chaudhury, A.M. (1998) Genetic control of male fertility in Arabidopsis thaliana: structural analyses of postmeiotic developmental mutants. Planta 205, 492-505.

Tusher, V.G., Tibshirani, R. and Chu, G. (2001) Significance analysis of microarrays applied to the ionizing radiation response. Proc Natl Acad Sci U S A 98, 5116-5121.

Van der Hoeven, R., Ronning, C., Giovannoni, J., Martin, G. and Tanksley, S. (2002) Deductions about the number, organization, and evolution of genes in the tomato genome based on 
analysis of a large expressed sequence tag collection and selective genomic sequencing. Plant Cell 14, 1441-1456.

van der Meer, I.M., Stam, M.E., van Tunen, A.J., Mol, J.N. and Stuitje, A.R. (1992) Antisense inhibition of flavonoid biosynthesis in petunia anthers results in male sterility. Plant Cell 4, 253-262.

Zhang, S. and Scheller, H.V. (2004) Photoinhibition of photosystem I at chilling temperature and subsequent recovery in Arabidopsis thaliana. Plant Cell Physiol 45, 1595-1602.

Zhou, N., Robinson, S.J., Huebert, T., Bate, N.J. and Parkin, I.A. (2007) Comparative genome organization reveals a single copy of $\mathrm{CBF}$ in the freezing tolerant crucifer Thlaspi arvense. Plant molecular biology 65, 693-705.

Zhu, J.K. (2001) Plant salt tolerance. Trends Plant Sci 6, 66-71. 


\section{Chapter 5}

Heat stress, male sterility and ABA:

A working hypothesis 


\begin{abstract}
The influence of heat stress on gene expression is ultimately reflected in plant growth and development. The occurrence of male sterility is known to be regulated by environmental factors including high temperatures and is correlated with changes in hormone levels, in particular abscisic acid (ABA). Stamenless-2 (sl-2) is a temperature sensitive mutant of tomato that exhibits typically high temperature related developmental abnormalities of the stamen under temperatures where wild type plants develop normally. Under standard growth conditions stamens of the $s l-2$ are sterile, shrunken in size, accumulate high ABA levels, fail to fuse laterally and produce naked ovules on their adaxial surface. Under higher heat stress, even carpel-like structures develop in place of stamens, indicating a homeotic transformation from stamens to carpels. It thus seems that there is a correlation between accumulation of high ABA levels, male sterility, organ identity and temperature. Our observations show that under moderate heat stress conditions several genotypes present various degrees of sterility reflected by anther malformations, including exerted or enlarged style, split anther cones or ectopic ovule-like structures on the adaxial anther surface. For example, in Moneymaker (MM), a heat sensitive genotype, most of the anthers developed entirely under long-term moderate heat stress (MHS) conditions exhibit malformations similar to sl-2. Anthers of N5024, a tolerant genotype, have very few or no malformed flowers at all under the same conditions. When ABA was applied in standard growth conditions to MM and N5024 developing meiotic anthers in order to see the effects of $\mathrm{ABA}$ on anther development and pollen fertility, no extreme malformations were observed except a slight increase in style length in the MM genotype. Furthermore, ABA application had similar results as heat stress with regards to pollen germination. In an attempt to correlate ABA dynamics and tolerance to heat in tomato meiotic anthers, we analysed the dynamics of $\mathrm{ABA}$ accumulation under heat stress in six tomato genotypes with contrasting responses to heat. The meiotic anthers of the tolerant genotypes maintained a lower level of ABA during the two weeks of heat stress, when compared to the anthers of the sensitive genotypes. Collectively this preliminary analysis supports the idea that a lower accumulation of ABA during heat stress is correlated with increased tolerance to heat in tomato developing anthers.
\end{abstract}




\section{Introduction}

Floral organs and reproductive development are particularly vulnerable to environmental stress, foremost amongst which is heat stress. The integrity of floral organ development plays a key role in the determination of crop yields. Male reproductive organ development is the most sensitive to environmental stress and flowers effected by high temperature frequently show male organ malformations, resulting in male sterility, such as malformed anthers, protruding styles, split anther cone, etc. (Kim et al., 2001; Lozano et al., 1998; Mittler, 2006; Polowick and Sawhney, 1985; Zinn et al., 2010). In plants, abscisic acid (ABA) often functions as a mediator of key phase-transitions throughout the plant life cycle. Several lines of evidence indicate basal levels of ABA, ABA synthesis and degradation and ABA signaling, exert a profound effect on plant growth, development and physiology (Arenas-Huertero et al., 2000; Cutler et al., 2010; Kotak et al., 2007). ABA appears to act as a promoter in plant growth and development (one important function is the regulation of plant respiration via stomatal closure), as well as an inhibitor in dormancy or abiotic stress responses (Sharp and LeNoble, 2002). At the molecular level, ABA activates a wide array of genes associated with high or low temperatures, drought, salinity, wounding, desiccation, storage proteins, dormancy, germination, the arrest of embryonic development, and the closure of stomata(Cutler et al., 2010)

With respect to the energy requirements of reproductive development and considering the correlation between $\mathrm{ABA}$ accumulation and assimilate distribution during fruit development, it was suggested that $\mathrm{ABA}$ also plays a role in the early development of reproductive plant organs (Peng et al., 2003; Zhang et al., 2001). For example, ABA biosynthesis occurs mainly in the parenchyma cells surrounding the anther vascular bundle sheath (Ji et al., 2011; Oliver et al., 2007). ABA has also been detected in nursing cells in A. thaliana (e.g. tapetum and integuments), which function in supplying nutrition for germ cell development (Peng et al., 2006). Thus, it appears that ABA metabolism, the response to abiotic stress, regulation of plant growth and development, are highly intertwined (Hey et al., 2010).

Under stress conditions including heat, ABA accumulation has been mostly assigned a negative function, often being associated with male sterility. ABA synthesis is one of the most rapid responses of plants to abiotic stress, by triggering ABA-inducible gene expression (Wilkinson and Davies, 2010). In plants ABA is often associated with inhibition of growth during abiotic or biotic stress conditions, as stressful conditions generally cause ABA accumulation (Saini and Aspinall, 1982; Wilkinson and Davies, 2010). Moreover, exogenous ABA applications inhibit plant growth and induce sterility (Davies, 2010). In tomato, exogenous ABA suppresses stamen development and causes pollen abortion both in vitro (Rastogi and Sawhney, 1987) and in vivo (Sekhar and Sawhney, 1991). Similarly, in rice, ABA treatment of the spike induces high levels of sterility (Ji et al., 2011). Currently, the role for ABA in reproductive development and abiotic stress tolerance of reproductive structures remains ambiguous (Zhang et al., 2006). A number of studies provide evidence that the beneficial or detrimental role(s) assigned to ABA accumulation during reproductive development are heavily dependent upon plant phenology and physiological context. However, recent studies propose excessive ABA accumulation negatively impacts the abiotic stress tolerance of reproductive tissues (Baron et al., 2012b; Chinnusamy et al., 2008; Ji et al., 2011; Sreenivasulu et al., 2012).

A molecular analysis in several crop plants revealed distinctive cellular and organ-level aspects of ABA homeostasis that distinguish stress-tolerant and stress-sensitive germplasm at the reproductive stage of development (Baron et al., 2012a). Comparison of ABA biosynthesis in coldsensitive and cold-tolerant rice lines indicated that cold-tolerant rice accumulated less ABA in response to cold treatment. This difference in ABA homeostasis appears to be due to both lower ABA biosynthesis and faster turnover of ABA (Oliver et al., 2007). When subjected to heat stress, developing anthers of two rice cultivars displayed a decrease in the contents of IAA, GAs, free proline and soluble proteins but an increase in the ABA content. Compared to the sensitive genotype, the 
tolerant retained significantly higher levels of free proline and GAs and a lower level of ABA, along with higher pollen vigour and pollen germination rate even after prolonged high temperature treatment. This study also suggests a possible correlation between pollen viability/floret sterility and high temperature-caused changes in endogenous ABA levels (Tang et al., 2008). Unlike droughtsensitive wheat varieties, drought-tolerant wheat accumulates less ABA, which correlates with lower ABA biosynthesis and higher ABA catabolic gene expression. Higher ABA levels in these lines cause increased sensitivity to drought stress and changes in the balance between ABA-synthesizing and degrading enzymes appear to be responsible for that (Ji et al., 2011).

Similarly, various genic male sterile tissues of a line of B. napus, including leaves, flowers and stamens have higher endogenous concentration of ABA in comparison to wild-type tissues. Stamens have the highest amount of ABA in comparison to other tissues. However, a role of other plant growth substances in male-sterility such as gibberellins, IAA and ethylene, has not been ruled out (Shukla and Sawhney, 1994). From these studies, excessive ABA accumulation within reproductive structures was proposed to negatively correlate with abiotic stress tolerance. Maintaining low ABA levels, therefore, is important for stress tolerance and pollen fertility. One important downstream effect of increasing $\mathrm{ABA}$ is the repression of sugar transport, and the maintenance of anther sink strength which is also critical for the development of fertile pollen (Ji et al., 2011).

In contrast to high temperature stress, when tomato (Solanum lycopersicum) plants are exposed to low temperatures, they produce flowers showing homeotic changes with alterations in the number, morphology, and pattern of fusion of floral organs. As a consequence, abnormal fruits of low economic value are produced from these flowers. The carpelloid stamens developing under low temperature show naked ovules on the internal side of the carpel sectors and it was proposed that the hometic changes taking place in the developing flower are also modulated via the hormonal levels (Lozano et al., 1998). These phenotypic changes show intriguing similarities to changes that occur under high temperature but it remains unclear to what extent the processes leading to the changes have the same causative basis at a molecular level.

Interestingly, stamenless-2 (sl-2), a tomato mutant, exhibits very similar homeotic modifications of the anthers, is temperature conditioned male sterile and it also contains high level of ABA in the sterile anthers. The increase in ABA level in sl-2 stamens coincided with first signs of abnormalities in the anthers. At low temperatures, male fertility is restored in $s l-2$ flowers (Sawhney, 1983 ) and this correlates with a drop in ABA to levels comparable to those in normal tissues. Also, $s l$ 2 flowers contain low levels of gibberellins but high levels of IAA, suggesting that male sterility in $\mathrm{sl}$ 2 is a manifestation of hormonal imbalance involving at least three hormones. In addition, another tomato male sterile mutant, also over-producing $\mathrm{ABA}$, presents flowers with short and malformed anthers. In the anthers of this mutant, microspore mother cells are differentiated but meiosis does not occur (Sheoran et al., 2009). This data suggests that normal stamen development in tomato takes place in the presence of low ABA levels (Singh and Sawhney, 1998).

In our experiments, heat sensitive tomato plants that are exposed to moderately high temperatures for an extended period exhibit a high percentage of flowers with homeotic changes and low pollen germination. However, when tolerant genotypes are grown under heat, they develop very few or no such malformed flowers and maintain good pollen germination. We tested the hypothesis that heat tolerance is associated with maintenance of low ABA levels and no anther malformations during heat stress for several tomato genotypes. 


\section{Materials and methods}

\section{Plant material and heat stress conditions}

Several tomato genotypes were used for this experiment: N5024, FR and HS1 were obtained from Nunhems Netherlands BV (the Netherlands), and are commercial varieties with different tolerances to heat. MM, Saladette and Pull were obtained from the seed repository of the Radboud University in Nijmegen. Seeds were germinated in potting compost in trays and transferred after two weeks to a growth chamber under standard temperature (ST) conditions, with a 16 -h light $\left(26^{\circ} \mathrm{C}\right) / 8$-h dark $\left(18^{\circ} \mathrm{C}\right)$ cycle. Fluorescent and incandescent lighting provided a photosynthetic photon flux density of 450-500 $\mu \mathrm{mol} \mathrm{m} \mathrm{sec}^{-1}$. The plants were watered daily and fertilized weekly. We have used six-week-old plants for all experiments. The heat treatment was initiated by progressively increasing the temperature from $26^{\circ}$ to $32^{\circ}$ over half an hour period, and samples were collected at $0 \mathrm{~h}, 2 \mathrm{~d}, 1$ week and 2 weeks of moderate heat stress (MHS). In order to describe the dynamics of hormonal responses of tomato developing meiotic anthers to MHS, the temperature range for the experiment was chosen based on agronomically relevant temperatures shown to have a significant effect on pollen viability (Sato et al., 2006), rather than using classical heat shock conditions of $42^{\circ}-45^{\circ} \mathrm{C}$ (Frank et al., 2009). Heat stress was applied to whole plants in the growth chamber according to the following scheme (Figure 1), under the same light conditions as stated above.

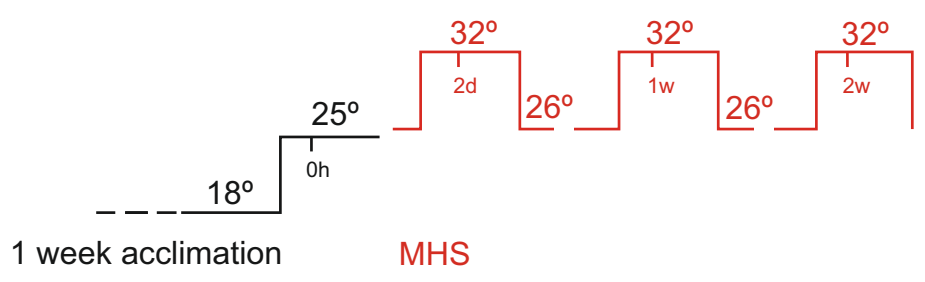

Figure 1. Plant heat-stress treatment scheme.

The lines represent the temperature of growth chamber during plant treatment (as described in the text). The black line represents standard conditions with the small vertical tick indicating the time of harvest. The red line represents MHS with the vertical ticks showing the different harvest time points. Gaps in lines indicate the continuation of the temperature cycle.

\section{ABA treatments}

Developing flower buds were sprayed with $100 \mu \mathrm{M}+/$ - ABA in $10 \%$ methanol. Control plants were treated with a solution containing $10 \%$ methanol. Plants were normally watered daily and fertilized weekly. The treatment has been performed daily for a period of two weeks, to follow flower bud development and morphology. Three bud clusters per plant were tagged for spraying, and 3 plants per genotype were used. After two weeks, fully open flowers were collected and assessed for changes in anther morphology and pollen germination. When necessary, plant material has been studied under the binocular.

\section{Pollen germination assay}

Two replicates of three anther cones of freshly opened flowers were cut and 500uL of pollen germination media containing $20 \mathrm{mM}$ MES pH6.0, $3 \mathrm{mM} \mathrm{Ca}\left(\mathrm{NO}_{3}\right)_{2}, 1 \mathrm{mM} \mathrm{KCl}, 0.8 \mathrm{mM} \mathrm{MgSO} 4,1.6$ $\mathrm{mM}$ boric acid, 2.5\% (w/v) sucrose and 24\% (w/v) PEG 4000 were added. After vortexing to release the pollen grains, incubation was done for 15 hours at room temperature, in a rotating shaker. Pollen germination was examined under a light microscope with the 10x objective. Scoring of the germination was performed in 5 frames. Mean germination percentages and SD were calculated and integrated. 


\section{Cytology}

Anther tissue with ectopic ovules was collected from flowers developed under MHS, bleached in $0.4 \%$ hypochlorite for 90 minutes and washed with sterile water. The anthers were fixed in FAA (3.7\% formaldehyde, $5 \%$ acetic acid, and 50\% ethanol) vacuum infiltrated for 15 minutes twice, and left overnight. Fixed tissues were dehydrated with ethanol, cleared with xylene and embedded in parafin (Paraplast, Sigma-Aldrich, Steinheim, Germany). Embedded tissues were sliced into $8 \mu \mathrm{m}$ sections. Paraplast was removed with xylene, sections were hydrated and stained in $4 \%$ toluidine blue staining (Acros, Belgium). Pictures were taken with 10 times objective.

\section{ABA extraction and quantification}

All samples were harvested in May-June between 11.00 and 13.00 hours and directly frozen in liquid nitrogen. Extraction, clean-up and analysis of ABA from MM, N5024, Pull and Saladette were carried out according to a modified protocol described by Müller et al. (2002). Circa $100 \mathrm{mg}$ of frozen plant material were transferred into a $1.5 \mathrm{ml}$ Eppendorf vial together with $990 \mu 1$ methanol, $10 \mu \mathrm{l}$ of internal standard $(1.03 \mathrm{pmol} / \mu \mathrm{l}(2 \mathrm{H}) 6-\mathrm{ABA})$ and 5 stainless steel balls with a diameter of $3 \mathrm{~mm}$. The samples were heated for $20 \mathrm{~min}$ at $60^{\circ} \mathrm{C}$ and subsequently disrupted for $20 \mathrm{~min}$ using a vibrating-ball micromill (Retsch MM301, Haan, Germany) at a vibration frequency of $30 \mathrm{~s}^{-1}$. After vortexing and incubation for $15 \mathrm{~min}$ at room temperature the extract was centrifuged for $10 \mathrm{~min}$ at $14000 \mathrm{~g}$. The residue was re-suspended in $300 \mu 1$ methanol, vortexed and after incubation for $15 \mathrm{~min}$ at room temperature centrifuged as described above. Pooled methanolic extracts were centrifuged, transferred into a new Eppendorf vial and reduced to dryness in a vacuum centrifuge (Savant SPD 111V, Thermo Fisher Scientific, Schwerte, Germany) at $40^{\circ} \mathrm{C}$ for $30 \mathrm{~min}$ at $320 \mathrm{mbar}$ and thereafter at $200 \mathrm{mbar}$. The dried sample was dissolved in $50 \mu 1$ methanol by using a vortex and a subsequent ultrasonic treatment for $5 \mathrm{~min}$. After short centrifugation $200 \mu$ l diethyl ether were added and closed tubes were vortexed and exposed to ultrasonic treatment followed by centrifugation as described above. The aminopropyl solid phase extraction column (Chromabond NH2 shorty $10 \mathrm{mg}$, Macherey-Nagel GmbH \& Co. KG, Düren, Germany) was equilibrated with $200 \mu$ diethyl ether, followed by application of the dissolved sample. The empty tube was flushed with $100 \mu 1$ diethyl ether, which was also applied on the column. Then the column was washed twice with $200 \mu$ l diethyl ether, three times with $200 \mu 1$ of a mixture of chloroform/2-propanol $(2: 1, \mathrm{v} / \mathrm{v})$, three times with $200 \mu \mathrm{l}$ chloroform and at last with $100 \mu$ diethyl ether. The analytes were eluted three times with $200 \mu$ diethyl ether containing $4 \%$ acetic acid. Combined eluates were reduced to dryness in a stream of nitrogen at room temperature, re-dissolved in $20 \mu 1$ methanol, methylated with $200 \mu 1$ ethereal diazomethane, taken to dryness again in a nitrogen stream and dissolved in $10 \mu 1$ ethyl acetate. Separation and mass fragment analysis were conducted using a Varian Saturn 2200 ion-trap mass spectrometer connected to a CP-3800 gas chromatograph fitted with a CombiPal autoinjector (Agilent, Santa Clara, California, USA). The GC settings were as follows: splitless injection $(1 \mu \mathrm{l})$ with $1 \mathrm{~min}$ pressure pulse at $24 \mathrm{psi}$; splitter opening 1:100 after $1 \mathrm{~min}$; columns: Phe-Sil retention gap $10 \mathrm{~m}$ x 0,32 mm ID, ZB-50 $50 \%$ Phenyl-50 \% Dimetylpolysiloxane $30 \mathrm{~m} \times 0,25 \mathrm{~mm}$ ID x $0.25 \mu \mathrm{m}$ film thickness, Phenomenex; carrier gas: $\mathrm{He}, 1 \mathrm{ml} \mathrm{min}^{-1}$, constant flow; temperature program: $1 \mathrm{~min}$ isothermally at $60^{\circ} \mathrm{C}$, followed by a linear ramp at a rate of $40^{\circ} \mathrm{C} \mathrm{min}-1$ to $150^{\circ} \mathrm{C}$, isothermally for $6 \mathrm{~min}$ at $150^{\circ} \mathrm{C}$, followed by linear ramp of $20^{\circ} \mathrm{C} \min ^{-1}$ to $250^{\circ} \mathrm{C}$; transfer line temperature $230^{\circ} \mathrm{C}$. The following MS settings were used: CI-MRM mode; positive ion detection; reactant gas methanol; temperatures of manifold and ion trap $60^{\circ} \mathrm{C}$ and $200^{\circ} \mathrm{C}$, respectively; axial modulation $4 \mathrm{~V}$; scan time $0.4 \mathrm{~s}$ scan-1; multiplier offset $300 \mathrm{~V}$; emission current $50 \mu \mathrm{A}$; maximum ionisation time $2 \mathrm{~ms}$; maximum reaction time $128 \mathrm{~ms}$; waveform: resonant. Settings for endogenous ABA were chosen as follows: parent ion $(\mathrm{m} / \mathrm{z})=261\left(\mathrm{M}-\mathrm{H}_{2} \mathrm{O}+\mathrm{H}\right)^{+}$, diagnostic product ion $(\mathrm{m} / \mathrm{z})=$ 229 , excitation amplitude $0.6 \mathrm{~V}$. A second channel analysing the isotopically labelled standard $(2 \mathrm{H}) 6-$ ABA used the parent ion $(\mathrm{m} / \mathrm{z})=267\left(\mathrm{M}-\mathrm{H}_{2} \mathrm{O}+\mathrm{H}\right)^{+}$and the diagnostic daughter ions $(\mathrm{m} / \mathrm{z})(2 \mathrm{H}) 6-\mathrm{ABA}$ 
$=233+234$. The amounts of endogenous ABA were calculated from the signal ratios of the unlabelled over the corresponding stable isotope-containing mass fragments.

Extraction, clean-up and analysis of ABA from FR and HS1 were carried out according to a modified protocol described by Vilaro et al. (2006). ABA was quantified in triplicates by GC-MS as described for GA analysis (Griffiths et al., 2006), but with modifications. Freeze-dried samples were homogenized in $50 \mathrm{ml} \mathrm{80 \%} \mathrm{(v/v)} \mathrm{methanol/water} \mathrm{in} 100 \mathrm{~mL}$-flasks after which $300 \mathrm{ng} 3$-methyl[2H3]ABA was added as internal standard. Samples were purified as described previously except that the pooled ethyl acetate phases after elution from the Varian Bond Elut $\mathrm{NH}_{2}$ cartridge $(100 \mathrm{mg}$; Kinesis, St. Neots, UK) were evaporated to dryness in vacuo, and dissolved in ethyl acetate $(20 \mathrm{~L})$ of which $2 \mathrm{~L}$ was injected into a TR-1 capillary column $(30 \mathrm{~m} \times 0.25 \mathrm{~mm} \times 0.25 \mathrm{~mm}$ film thickness; Thermo Fisher Scientific, Madison, USA) at $50^{\circ} \mathrm{C}$. The split valve (50:1) was opened after 2 min and the temperature increased at $20^{\circ} \mathrm{C} / \mathrm{min}$ to $180^{\circ} \mathrm{C}$ and then at $4^{\circ} \mathrm{C} / \mathrm{min}$ to $300^{\circ} \mathrm{C}$. The instrument was operated in selective ion monitoring mode, monitoring the ions at m/z 190 and 162 for ABA and 193 and 166 for [2H3]ABA. The amount of ABA was determined from the peak areas for the ions $\mathrm{m} / \mathrm{z} 190$ and 193 by reference to a calibration curve. 


\section{Results and discussion}

Observations on the stamenless-2 (sl-2) mutant of tomato show that this genotype is hypersensitive to elevated temperatures (Sing et al. 1998). The phenotype is exhibited under temperatures $\left(25^{\circ} \mathrm{C}\right)$ where wild type tomatoes flower normally and in $s l-2$ results in deformed anthers, exerted style, formation of ectopic ovules on the adaxial surface of the anthers and low pollen viability (Figure $2 \mathrm{~d}-\mathrm{f}$ ). At low temperatures $\left(15^{\circ} \mathrm{C}\right)$, the $s l-2$ mutant displays a normal floral morphology. These characteristics are phenocopied by heat sensitive genotypes under high temperature $\left(32^{\circ} \mathrm{C}\right.$ daytime, $26^{\circ} \mathrm{C}$ night $)$ where pollen germination levels are also compromised (Figure 21 ).

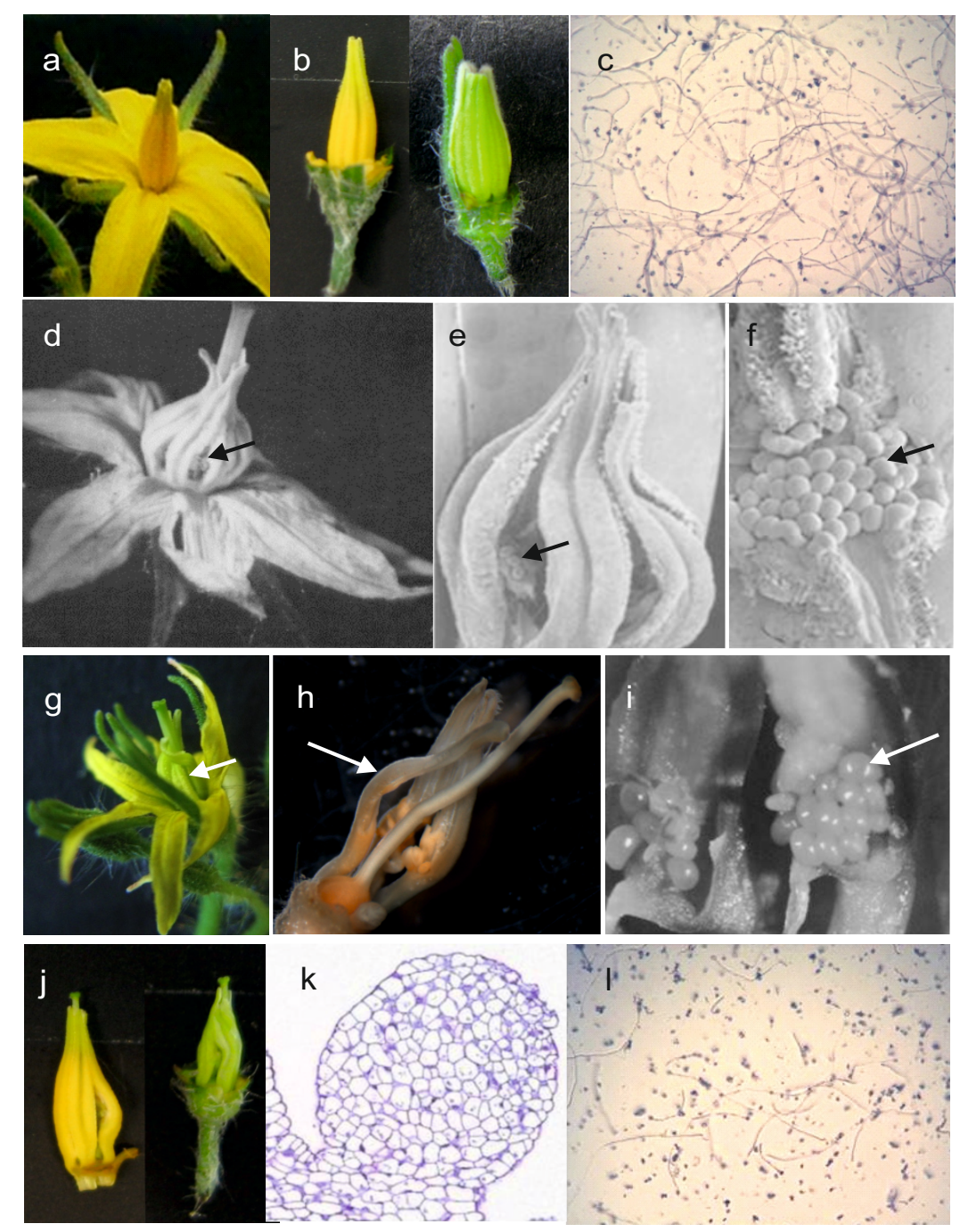

Figure 2. Phenotypic comparison of the MM genotype with the $s l-2$ mutant. Flower (a), anthers (b) and pollen germination (c) of MM under standard conditions. Flower (d), anther (e) and close-up of the anther cone (f) of the $s l-2$ mutant under standard conditions. The arrows indicate the location of ectopic ovules. (d-f; after Sawhney 1973). Flower development of MM under heat treatment (g-l). Arrow indicates the deformed anther cones (g). Enlarged view of a partially dissected flower (anther cone and female organs) revealing ectopic ovules. Arrow indicates anther deformation (h). Note the exuded style in (g) and (h). Micrograph of ectopic ovules on the anther (i). Deformation and splitting of the anther cone in MM flowers under heat-stress conditions is similar to the $s l-2$ mutant under standard conditions (j). In a section through an ectopic MM ovule, no differentiated tissues can be found (k). Reduced MM pollen germination under heat-stress conditions (1).

Although the mutation in the $s l-2$ cannot be directly linked to ABA, a characteristic of the mutant is a very high level of $\mathrm{ABA}$ in the anthers after transfer to non-permissive temperature and 
highly correlated with the observed morphological aberrations (Singh et al., 1992). In order to investigate the link between $\mathrm{ABA}$, heat stress and aberrant morphogenesis of generative tissues we compared a range of tomato genotypes under normal conditions $\left(26^{\circ} \mathrm{C}\right.$ day, $18^{\circ} \mathrm{C}$ night $)$ and under heat stress. The heat stress applied in these experiments, constitute a moderate heat stress (MHS) of 32 ${ }^{\circ} \mathrm{C}$ during the day combined with $26^{\circ} \mathrm{C}$ during the night. Under these conditions the commercial cultivar Moneymaker (MM) responds with a typical heat sensitive macroscopic phenotype showing exerted style and anther deformation (Figure $2 \mathrm{~g}$ ). Furthermore, MM also produces ectopic ovules on the inside of the anther cone similar to the $s l-2$ mutant (Figure $2 \mathrm{~h}$, i). Sections through such ectopic ovules (Figure $2 \mathrm{k}$ ) show that these are devoid of differentiated generative tissues and cell types found in normal ovules. The pollen formed in the MM heat stressed tissues also has a lower viability (Figure $21)$.

In a commercial genotype, selected for resistance to high temperature, N5024 (Nunhems BV), treatment with elevated temperature $\left(32^{\circ} \mathrm{C}\right.$ day, $26^{\circ} \mathrm{C}$ night $)$ results in only slight aberrant morphological deviations (Figure 3 a) and no appearance of ectopic ovules (Figure $3 \mathrm{~b}, \mathrm{c}$ ). In addition, the effects of the high temperature appear to have a less pronounced effect on pollen viability in in vitro germination assays (Figure $3 \mathrm{~d}, \mathrm{e}$ ).

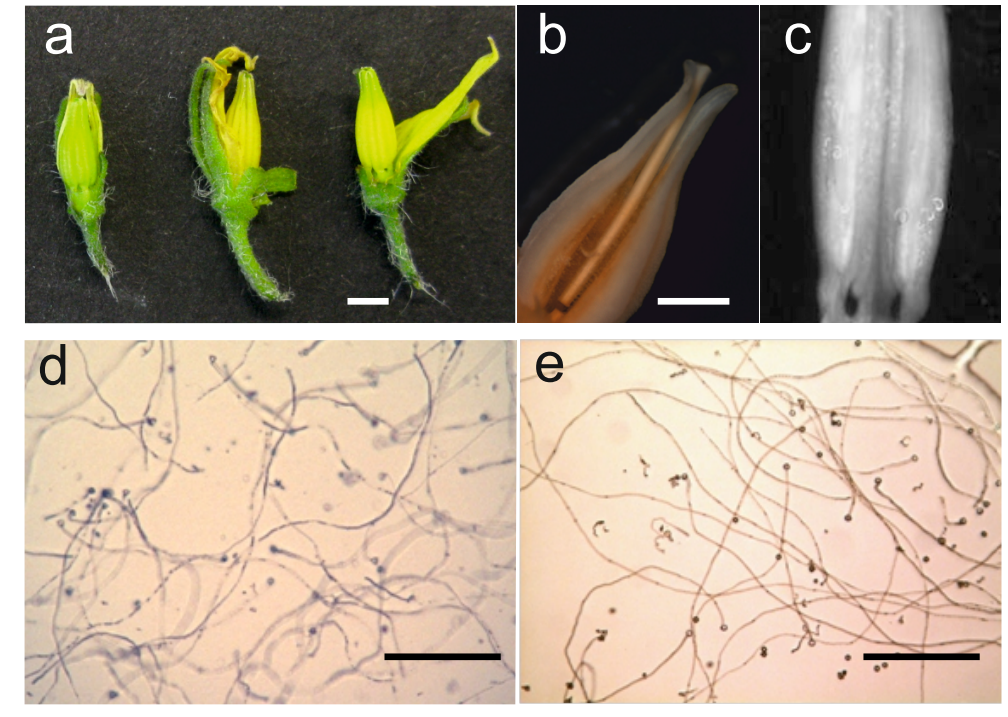

Figure 3. Heat stress phenotype of the tolerant N5024 genotype. a. Flower development under heat treatment. b. Partially dissected anther cone with normal anthers and non-exerted style. c. Micrograph of N5024 anther cone. d. N5024 pollen germination under standard conditions e. Slightly reduced pollen germination under heat treatment. Scale bars in a-c represent $1 \mathrm{~mm}$ and in $\mathrm{d}$ and e $50 \mu \mathrm{m}$.

In order to establish a link between the phenotypic abnormalities of heat stressed generative tissues (anthers and styles) and the high ABA levels that were found in the $s l-2$ mutant, developing buds of both sensitive (MM) and resistant genotypes (N5024) were morphologically analysed as described above and were treated with ABA in the form of a spray. A concentration of $100 \mu \mathrm{M} \mathrm{ABA}$ dissolved in $10 \%$ methanol was used in these experiments and the same solution without ABA was used as a control. The results of the ABA treatment clearly show a partial replication of the heat-stress symptoms (Figure 4). The main morphological effect of ABA treatment is the exerted style in the sensitive genotype MM (Figure $4 \mathrm{c}, \mathrm{d}$ ). Some deformation of the anther cone can also be observed, particularly at the tips of the anthers where a separation and curling of the distal ends of the anthers can be observed (Figure 4 d). Interestingly, the more resistant genotype N5024 does not display these phenotypic alternations (Figure $4 \mathrm{a}, \mathrm{b}$ ). The effects of ABA treatment on pollen germination in an in vitro assay also parallels the heat-stress phenotype with a much lower pollen viability in the heat sensitive genotype MM compared with tolerant genotype (Figure 4 e). A numeric assessment of the 
germination rates of pollen in the genotypes is shown in Figure 21 where the dramatic effect of ABA treatment can be observed on the sensitive genotype.
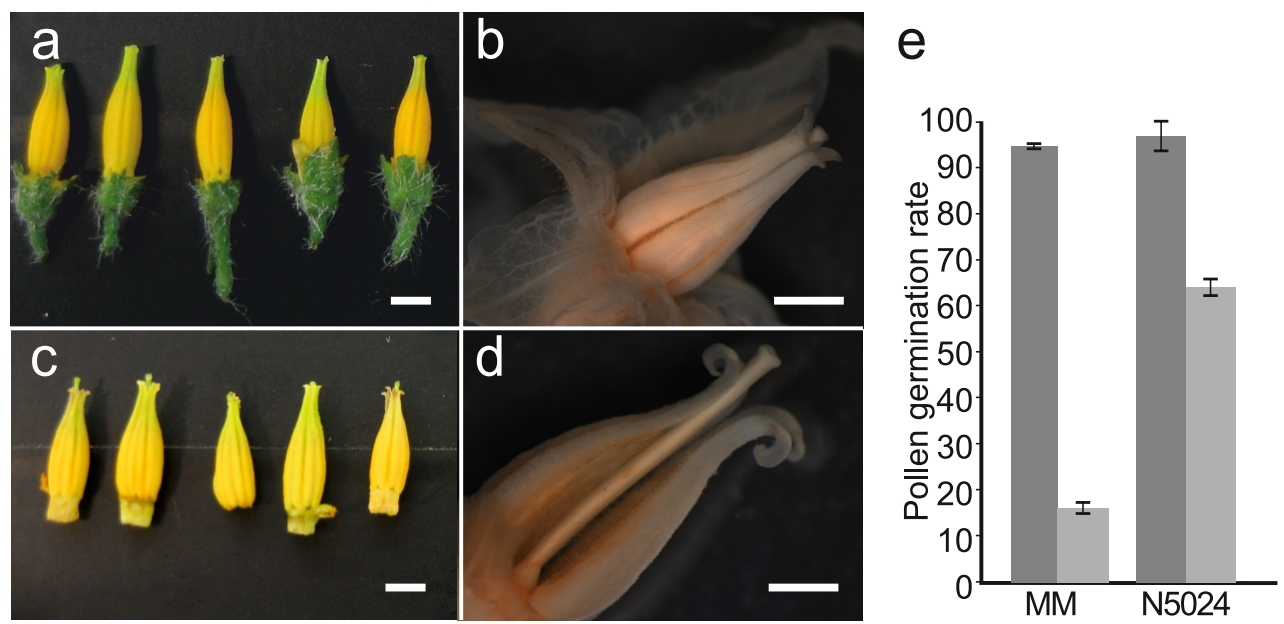

Figure 4 Phenotypic effects of $\mathrm{ABA}$ treatment under standard conditions in tolerant and sensitive tomato genotypes. Anther cones of the tolerant genotype N5024, treated with ABA (a). Close-up of whole flower of N5024 showing normal morphology (b). Anther cones of MM treated with ABA showing apical splitting and exerted styles (c). Close-up of MM anther cone after ABA treatment with apical anther curling and partially exerted style (d). Pollen germination statistics from ABA treated anthers of MM and N5024 (light grey bars), compared to the non-treated controls (dark grey bars). Error bars represent the standard error of the mean (e). Size bars in a-d are $2 \mathrm{~mm}$.

Having established a link between heat stress and ABA levels regarding a subset of the phenotypic alterations, we analysed an additional set of genotypes for ABA content under heat stress. For this experiment a set of genotypes was chosen with a wider range of heat tolerance and susceptibility phenotypes in order to establish a clearer link between heat stress and ABA levels. Although we have established that major gene regulation changes occur in the first hours of heat stress (Chapter 3 of this thesis), the morphological and cytological changes occur over a much longer period of time. Thus, the analysis of ABA levels was undertaken over an extended time period, ranging from 2 days heat treatment to two weeks. The heat stress applied for this experiment conforms to the temperature regime used for the morphological analysis described above $\left(32^{\circ} \mathrm{C}\right.$ day, $26^{\circ} \mathrm{C}$ night $)$. Comparing the more heat resistant N5024 genotype with MM, the indication is that ABA levels in the sensitive genotype rise dramatically on extended heat treatment showing a nearly 17 fold increase after 2 weeks of heat treatment. In the more tolerant genotype N5024 there is only a 4-fold increase (Figure 5). Two other genotypes with intermediate levels of tolerance to heat (Pull and HS1) show an insignificant change in ABA levels in the course of the experiment compared to the more sensitive genotype FR. These results indicate a general correlation between high relative levels of ABA after 2 weeks of heat stress and sensitivity to heat in the generative tissues resulting in low pollen germination. A sixth genotype included in this analysis (Saladette) which corresponds to an heirloom variety with morphological differences in leaf shape and growth habit but a high level of heat stress tolerance, shows a net 3-fold reduction of ABA levels over the two weeks. Although the initial levels of ABA are unusually high in anthers of this genotype, the general trend is to accumulate lower relative levels of $\mathrm{ABA}$ at the end of the experimental period compared to the initial stages in the more tolerant genotypes, and higher levels in the sensitive genotypes. However, the insufficient number of genotypes tested and the lack of a replicate experiment makes it difficult to establish a significant correlation between absolute ABA levels and time/treatment- dependent fluctuations. This is particularly true for one genotype (Saladette) that appears to be an exceptional case. 

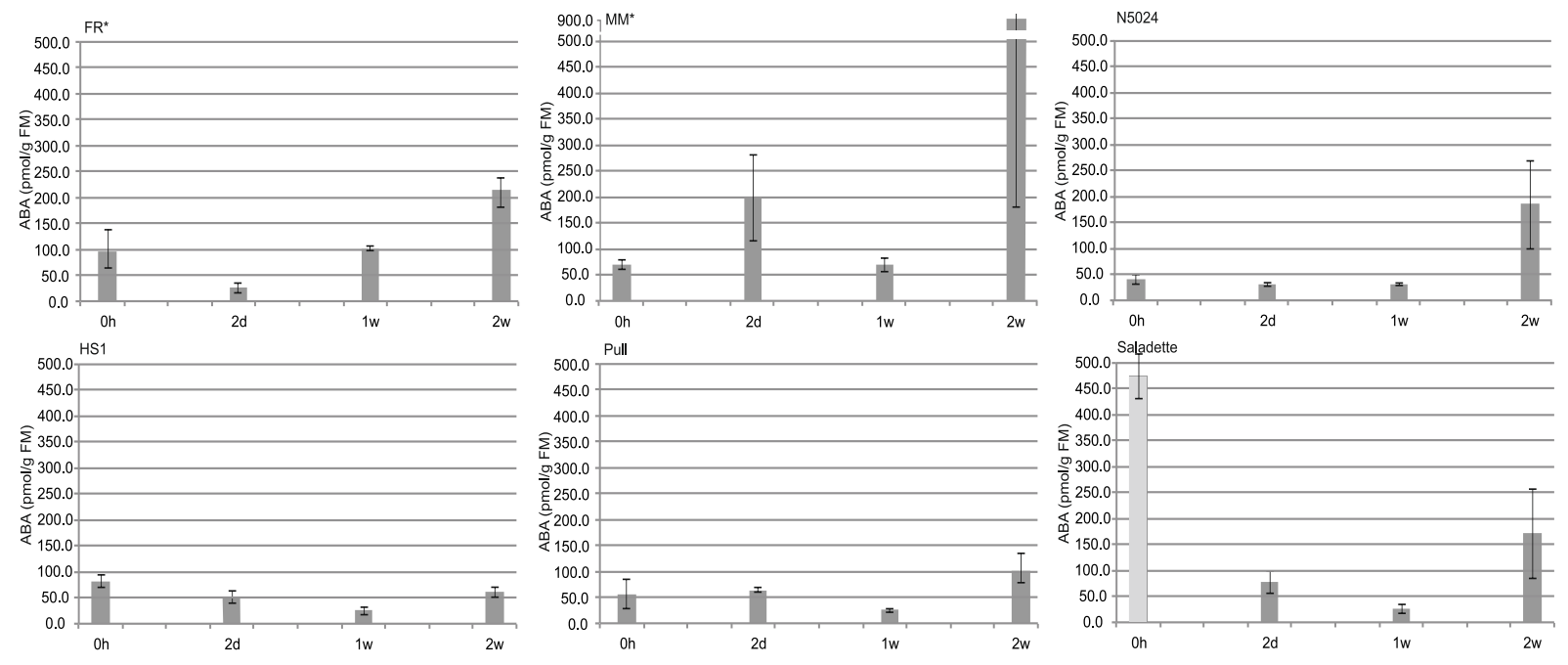

Figure 5 Dynamics of ABA levels in 6 tomato genotypes with different levels of heat tolerance under the influence of MHS. ABA levels (pmol/g FW) were determined at 3 time points (2 days; $2 \mathrm{~d}, 1$ week; $1 \mathrm{w}$ and 2 weeks; $2 \mathrm{w}$ ) after MHS with one sample taken prior to heat treatment (0h). The error bars represent the standard error of the mean of three samples. In Saladette the ABA level at time point $0 \mathrm{~h}$ is inconsistent with the observations in the other genotypes and the bar has thus been shaded in light grey.

As a method of assessing the sensitivity to high temperature, we also made measurements of pollen viability by in vitro germination assays of all the genotypes used in the ABA measurements. In all genotypes, pollen germination of plants under standard conditions was between $80 \%$ and $95 \%$ (Figure 6). We ranked genotypes according to the levels of pollen germination under high temperature conditions and this ranking corresponds to the generally observed levels of fruit set under high temperature in the field as communicated by the breeders and our own observations from green house experiments (data not shown). The genotypes with low pollen germination levels ( $<50 \%$; FR and MM) are also the most sensitive genotypes while the more tolerant genotypes show higher germination rates.

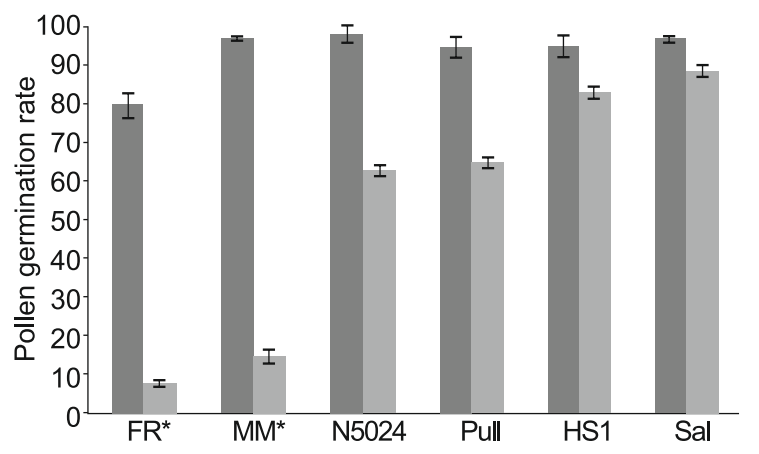

Figure 6. Pollen germination levels of sensitive and tolerant genotypes after MHS. Using the same genotypes where ABA levels were measured, pollen germination rates after 2 weeks of MHS were determined as an indication of heat tolerance. Dark grey bars are the non-treated controls and the light grey bars show the pollen germination rates after treatment. The data represents the average of 5 independent germination trials, with the error bars corresponding to the standard error of the means.

ABA is chemically derived from carotenoids and belongs to a set of signal compounds that includes the strigolactones and are more distantly related to invertebrate juvenile and animal retinoic acid terpenoid hormones. Thus, ABA and related compounds are widespread throughout the evolutionary 
tree and represent ancient stress response molecules (Hauser et al., 2011). The best-defined role for $\mathrm{ABA}$ is as a coordinator of stress responses. ABA levels increase as a result of abiotic stresses such as heat, UV light, cold, drought and salinity. The function of ABA in regulating stomatal guard cell opening is well documented, however, the more subtle effects that link to reproductive development are less well understood (Sreenivasulu et al., 2012). Our results presented here show that the phenotypic effects of ABA treatment mimic the effects of high temperature stress providing evidence of common signalling pathways.

Throughout evolution, the male generative cell development is susceptible to stress. Both spermatogenesis and pollen mother cell development are inhibited by elevated temperature. Although the mechanisms of how $\mathrm{ABA}$ interacts with the heat stress response in plants remains unclear, the early events in pollen development and the abortion pollen mother cell maturation are intimately linked to ABA (Razem et al., 2006). Both the study of ABA application and the phenotypic characterisation of the ABA mutant $s l-1$, confirm this relationship. The regulation of carbohydrate metabolism by ABA has been well documented (Kempa et al., 2008). Mitochondrial lesions leading to male sterility highlight the requirement of carbohydrates and general cellular energy flux during pollen development. It is possible that ABA signalling is a key process that differentiates heat sensitive and tolerant genotypes in tomato, with regards to reproductive development.

We conclude from these results that the morphological changes in the floral tissues and the overall changes in ABA levels are correlated with increased temperature sensitivity. Furthermore, the variation in the initial levels of $\mathrm{ABA}$ in the tolerant and sensitive genotypes are inconsistent, with a very high initial concentration of $\mathrm{ABA}$ in the most tolerant genotype (Saladette) compared to a relatively low initial level in N5024 which is also tolerant to heat stress. It appears thus that there is a weak correlation between sensitivity to heat and changes in the ABA accumulation pattern. This may indicate a different mechanism of ABA turnover in these genotypes and suggests that the absolute ABA levels after two weeks of heat treatment are more informative, while constitutive ABA levels might not be relevant for tolerance to heat. Clearly more research is needed to resolve these issues.

\section{Acknowledgements}

Uwe Druege (Leibniz Institute of Vegetable and Ornamental Crops (IGZ), Erfurt, Germany) for ABA extraction and analysis.

Tatsiana Charnikhova (WUR, Wageningen) for ABA extraction and analysis.

Gerard van der Weerden for technical support with the climate chamber and greenhouse. 


\section{References}

Arenas-Huertero, F., Arroyo, A., Zhou, L., Sheen, J. and Leon, P. (2000) Analysis of Arabidopsis glucose insensitive mutants, gin5 and gin6, reveals a central role of the plant hormone ABA in the regulation of plant vegetative development by sugar. Genes \& development 14, 2085-2096.

Baron, K.N., Schroeder, D.F. and Stasolla, C. (2012a) Transcriptional response of abscisic acid (ABA) metabolism and transport to cold and heat stress applied at the reproductive stage of development in Arabidopsis thaliana. Plant Science 188, 48-59.

Baron, K.N., Schroeder, D.F. and Stasolla, C. (2012b) Transcriptional response of abscisic acid (ABA) metabolism and transport to cold and heat stress applied at the reproductive stage of development in Arabidopsis thaliana. Plant Science 188, 48-59.

Chinnusamy, V., Gong, Z.Z. and Zhu, J.K. (2008) Abscisic acid-mediated epigenetic processes in plant development and stress responses. Journal of integrative plant biology 50, 1187-1195.

Cutler, S.R., Rodriguez, P.L., Finkelstein, R.R. and Abrams, S.R. (2010) Abscisic acid: emergence of a core signaling network. Annual review of plant biology 61, 651-679.

Davies, P.J. (2010) The plant hormones: their nature, occurrence, and functions. In: Plant hormones pp. 1-15. Springer.

Frank, G., Pressman, E., Ophir, R., Althan, L., Shaked, R., Freedman, M., Shen, S. and Firon, N. (2009) Transcriptional profiling of maturing tomato (Solanum lycopersicum L.) microspores reveals the involvement of heat shock proteins, ROS scavengers, hormones, and sugars in the heat stress response. Journal of experimental botany 60, 3891-3908.

Griffiths, J., Murase, K., Rieu, I., Zentella, R., Zhang, Z.-L., Powers, S.J., Gong, F., Phillips, A.L., Hedden, P. and Sun, T.-p. (2006) Genetic characterization and functional analysis of the GID1 gibberellin receptors in Arabidopsis. The Plant Cell Online 18, 3399-3414.

Hauser, F., Waadtl, R. and Schroeder, J.I. (2011) Evolution of Abscisic Acid Synthesis and Signaling Mechanisms. Current Biology 21, R346-R355.

Hey, S.J., Byrne, E. and Halford, N.G. (2010) The interface between metabolic and stress signalling. Annals of botany 105, 197-203.

Ji, X., Dong, B., Shiran, B., Talbot, M.J., Edlington, J.E., Hughes, T., White, R.G., Gubler, F. and Dolferus, R. (2011) Control of abscisic acid catabolism and abscisic acid homeostasis is important for reproductive stage stress tolerance in cereals. Plant physiology 156, 647-662.

Kempa, S., Krasensky, J., Dal Santo, S., Kopka, J. and Jonak, C. (2008) A central role of abscisic acid in stress-regulated carbohydrate metabolism. PloS one 3, e3935-e3935.

Kim, S.Y., Hong, C.B. and Lee, I. (2001) Heat shock stress causes stage-specific male sterility in Arabidopsis thaliana. Journal of plant research 114, 301-307.

Kotak, S., Larkindale, J., Lee, U., von Koskull-Döring, P., Vierling, E. and Scharf, K.D. (2007) Complexity of the heat stress response in plants. Current opinion in plant biology 10, 310316.

Lozano, R., Angosto, T., Gomez, P., Payan, C., Capel, J., Huijser, P., Salinas, J. and Martinez-Zapater, J.M. (1998) Tomato flower abnormalities induced by low temperatures are associated with changes of expression of MADS-Box genes. Plant physiology 117, 91-100.

Mittler, R. (2006) Abiotic stress, the field environment and stress combination. Trends in plant science 11, 15-19.

Müller, A., Düchting, P. and Weiler, E.W. (2002) A multiplex GC-MS/MS technique for the sensitive and quantitative single-run analysis of acidic phytohormones and related compounds, and its application to Arabidopsis thaliana. Planta 216, 44-56.

Oliver, S.N., Dennis, E.S. and Dolferus, R. (2007) ABA regulates apoplastic sugar transport and is a potential signal for cold-induced pollen sterility in rice. Plant and Cell Physiology 48, 13191330.

Peng, Y.B., Lu, Y.F. and Zhang, D.P. (2003) Abscisic acid activates ATPase in developing apple fruit especially in fruit phloem cells. Plant Cell and Environment 26, 1329-1342.

Peng, Y.B., Zou, C., Wang, D.H., Gong, H.Q., Xu, Z.H. and Bai, S.N. (2006) Preferential localization of abscisic acid in primordial and nursing cells of reproductive organs of Arabidopsis and cucumber. New Phytologist 170, 459-466. 
Polowick, P.L. and Sawhney, V.K. (1985) Temperature Effects on Male-Fertility and Flower and Fruit-Development in Capsicum-Annuum-L. Scientia Horticulturae 25, 117-127.

Rastogi, R. and Sawhney, V.K. (1987) The Role of Plant-Growth Regulators, Sucrose and Ph in the Development of Floral Buds of Tomato (Lycopersicon-Esculentum Mill) Cultured Invitro. Journal of plant physiology 128, 285-295.

Razem, F.A., Baron, K. and Hill, R.D. (2006) Turning on gibberellin and abscisic acid signaling. Current opinion in plant biology 9, 454-459.

Saini, H.S. and Aspinall, D. (1982) Sterility in Wheat (Triticum-Aestivum L) Induced by Water Deficit or High-Temperature - Possible Mediation by Abscisic-Acid. Aust J Plant Physiol 9, 529-537.

Sato, S., Kamiyama, M., Iwata, T., Makita, N., Furukawa, H. and Ikeda, H. (2006) Moderate increase of mean daily temperature adversely affects fruit set of Lycopersicon esculentum by disrupting specific physiological processes in male reproductive development. Annals of botany 97, 731-738.

Sawhney, V.K. (1983) Temperature Control of Male-Sterility in a Tomato Mutant. J Hered 74, 51-54.

Sawhney, V.K. and Greyson, R.I. (1973) Morphogenesis of the stamenless-2 mutant in tomato. I. Comparative description of the flowers and ontogeny of stamens in the normal and mutant plants. American Journal of Botany, 514-523.

Sekhar, K.N.C. and Sawhney, V.K. (1991) Role of Aba in Stamen and Pistil Development in the Normal and Solanifolia Mutant of Tomato (Lycopersicon-Esculentum). Sexual Plant Reproduction 4, 279-283.

Sharp, R.E. and LeNoble, M.E. (2002) ABA, ethylene and the control of shoot and root growth under water stress. Journal of experimental botany 53, 33-37.

Sheoran, I.S., Ross, A.R.S., Olson, D.J.H. and Sawhney, V.K. (2009) Differential expression of proteins in the wild type and 7B-1 male-sterile mutant anthers of tomato (Solanum lycopersicum): A proteomic analysis. Journal of proteomics 71, 624-636.

Shukla, A. and Sawhney, V.K. (1994) Abscisic-Acid - One of the Factors Affecting Male-Sterility in Brassica-Napus. Physiologia plantarum 91, 522-528.

Singh, S. and Sawhney, V. (1998) Abscisic acid in a male sterile tomato mutant and its regulation by low temperature. Journal of experimental botany 49, 199-203.

Singh, S., Sawhney, V. and Pearce, D. (1992) Temperature effects on endogenous indole-3-acetic acid levels in leaves and stamens of the normal and male sterile 'stamenless- 2 ' mutant of tomato (Lycopersicon esculentum Mill.). Plant, cell \& environment 15, 373-377.

Sreenivasulu, N., Harshavardhan, V.T., Govind, G., Seiler, C. and Kohli, A. (2012) Contrapuntal role of ABA: Does it mediate stress tolerance or plant growth retardation under long-term drought stress? Gene 506, 265-273.

Tang, R.S., Zheng, J.C., Jin, Z.Q., Zhang, D., Huang, H. and Chen, L.G. (2008) Possible correlation between high temperature-induced floret sterility and endogenous levels of IAA, GAs and ABA in rice (Oryza sativa L.). Plant Growth Regulation 54, 37-43.

Vilaró, F., Canela-Xandri, A. and Canela, R. (2006) Quantification of abscisic acid in grapevine leaf (Vitis vinifera) by isotope-dilution liquid chromatography-mass spectrometry. Analytical and bioanalytical chemistry 386, 306-312.

Wilkinson, S. and Davies, W.J. (2010) Drought, ozone, ABA and ethylene: new insights from cell to plant to community. Plant Cell and Environment 33, 510-525.

Zhang, D.P., Chen, S.W., Peng, Y.B. and Shen, Y.Y. (2001) Abscisic acid-specific binding sites in the flesh of developing apple fruit. Journal of experimental botany 52, 2097-2103.

Zhang, J.H., Jia, W.S., Yang, J.C. and Ismail, A.M. (2006) Role of ABA in integrating plant responses to drought and salt stresses. Field Crops Research 97, 111-119.

Zinn, K.E., Tunc-Ozdemir, M. and Harper, J.F. (2010) Temperature stress and plant sexual reproduction: uncovering the weakest links. Journal of experimental botany 61, 1959-1968. 
Chapter 6

General discussion 


\section{Heat stress effects on flowering crop plants}

The increasing threat of climate change is already having a substantial impact on agricultural production worldwide as heat waves cause significantly yield losses with great risks for future global food security (Christensen and Christensen, 2007). Climatological extremes including very high temperatures are predicted to have a general negative effect on plant growth and development, potentially leading to catastrophic loss of crop productivity and resulting in wide spread famine. Future agricultural production and thus global food security will encounter additional challenges from human population growth, and populations from developing countries are likely to be the most seriously affected as nearly $50 \%$ rely entirely on agriculture and $75 \%$ of the world's poor live in rural areas. As a consequence, crop production will have to be tailored to sustain food security and it has been suggested that world food production will have to increase by $70 \%$ to meet the demand of an expected population of 9 billion in 2050. The growing food demand together with the treat of heavy crop losses due to global climate change imposes the urgent development of strategies for very significant improvements in food availability, among which enhancing crop productivity plays an important role.

Similar to many other crop species, tomato also displays marked responses to heat (Abiko et al, 2005; Porch \& Jahn, 2001). Hot summers in many agricultural regions negatively affect the vegetative and reproductive growth phases and result in up to $70 \%$ tomato harvest losses (Sato et al, 2002). The susceptibility to high temperatures in plants varies with the stage of plant development, heat stress affecting to a certain extent all vegetative and reproductive stages. The observed effects depend on species and genotype, with abundant inter- and intra-specific variations (Barnabás et al., 2008; Sakata and Higashitani, 2008). Crop plants exposed to an excess of heat of at least $5{ }^{\circ} \mathrm{C}$ above their optimal growing conditions exhibit a characteristic set of cellular and metabolic responses required for the plants to survive under the high-temperature conditions (Guy, 1999). These reactions include a decrease in the synthesis of normal proteins and the accelerated transcription and translation of heat shock proteins (HSPs) (Bray, 2000), the production of phytohormones such as abscisic acid (ABA) and antioxidants (Maestri et al., 2002) and changes in the organization of cellular structures, including organelles, and membrane functions (Weis and Berry, 1988). Heat stress induces changes in respiration and photosynthesis and thus leads to a shortened life cycle and diminished plant productivity (Barnabás et al., 2008). With respect to reproductive success, a decline of photosynthesis will eventually result in limited resource availability for reproduction in parental and gametophytic tissues due to a reduction in energy reserves leading to plant starvation (Sumesh et al., 2008; Young et al., 2004).

The male gametophyte is particularly sensitive to high temperatures at all stages of development, while the pistil and the female gametophyte are considered to be more tolerant. In tomato anthers developing under high temperature $\left(30^{\circ}-35^{\circ} \mathrm{C}\right)$, cell-proliferation is arrested and chloroplast or mitochondrial abnormalities occur (Sakata and Higashitani, 2008). In addition to the aborted development of pollen in early stages of anther development, continued high temperatures during later stages of anthesis result in poor anther dehiscence due to closure of the locules and decreased pollen tube germination and growth, ultimately disrupting fertilization and decreasing the seed and fruit number (Peet et al., 2002; Upadhyaya et al., 2011). In the research described in this thesis, we focused on the effects of moderate heat stress (MHS) rather than heat shock conditions on anther development. It is interesting to note that the changes in phenotype and transcriptome responses were actually similar to those found under heat shock conditions (Frank et al., 2009), indicating that similar processes take place. Whether the inducing signals of these responses are similar remains to be unknown.

Heat stress tolerance is a polygenic character often measured using complex traits such as yield under stress, which implicate important acclimation mechanisms that maintain the key functions 
of respiration and photosynthesis include rapid alterations in gene expression, increases in heat shock protein (HSP) levels, modification of membranes, alterations in the cytoskeleton and management of oxidative stress(Kotak et al, 2007). A survey of studies in tomato, rice and cowpea, shows that in most cases the tolerance was based on multiple, small-to-medium effect loci, each explaining a few to maximum $\sim 25 \%$ of the phenotype (Lin et al., 2010) (Grilli, 2007). This may indicate that heat tolerance as trait has many genetic determinants, which also agrees with reports that many different genetic modifications can artificially increase heat tolerance (Grover et al., 2013). Importantly, QTL positions have even been found to vary between studies using the same tolerant parental accession, which suggests an interaction with other genes in the background or high sensitivity to the assay parameters (Jagadish et al., 2010)

Various physiological injuries have been observed in plants growing under elevated temperatures, which consequently lead to decreased plant productivity (Vollenweider \& GünthardtGoerg, 2005). Investigations to identify floral characteristics affected by high temperatures in tomato reveal many abnormalities in anther development, especially in the tissues with important roles in pollen development and release, such as tapetum, stomium and circular cell clusters. (Lohar and Peat, 1998). The reduction in fruit set of tomato plants under high temperature was a result of disruptions in hormonal balance, sugar metabolism or proline transport during the critical phase of male gametophyte development (Sato et al., 2006; Singh and Sawhney, 1998). In Chapter 5 we illustrate various anther phenotype changes in a series of genotypes that show varying degrees of tolerance and susceptibility to high temperature and try to correlate these changes with phytohormone levels.

The work presented here has been performed as an attempt to gain insight into the response to heat in tomato meiotic anthers with contrasting responses to moderate heat stress. Here I summarize the main outcomes of our study and discuss potential opportunities to improve plant breeding under a continuously warming climate.

\section{Profiling the response to heat in developing anthers}

Targeted breeding for stress tolerance requires efficient screening procedures to identify key traits in diverse donor or tolerant lines and the understanding of the inheritance and molecular genetics of these traits. Abiotic stresses have a negative impact on plant development reflected by changes at molecular level. Thus, a stressful condition will be experimented by the plant via changes in the abundance of numerous transcripts and proteins through regulation of both gene expression and protein turnover. As a consequence, transcriptional and post-transcriptional regulations both play an essential role in the adaptation of cellular functions to the environmental changes. In the case of tomato, where the male gametophyte is the most sensitive to stress, genome-wide strategies are being used to investigate various aspects of pollen development, including responses to high temperatures (Bita et al., 2011; Frank et al., 2009; Jagadish et al., 2010). Molecular genetic markers such as AFLPs are an example of an effective tool that can be used to analyze plant genomes and understand how heritable traits associate with underlying genetic variations. Molecular markers often complement a modern plant genetic analysis when combined with genome-wide transcriptome analysis systems, e.g. cDNA microarray technology, regardless the studies are on model or non-model plant species (Hegarty et al., 2005).

Both cDNA-AFLP and Combimatrix microarray technology have been applied in this study to obtain a global overview of gene expression that is part of the response to moderate heat stress of anthers with contrasting sensitivities to moderate heat stress. While cDNA-AFLP allows the rapid interrogation of gene transcription without detailed sequence information, microarray technology has the advantage of simple massively parallel screening of transcriptional changes. We have used a variant of cDNA-AFLP (Bachem et al., 2008), which theoretically allows every transcript to be monitored, however, due to the difficulties in transcript-derived fragments identification from gel- 
isolated bands, only a subset could be practically be visualized. We used an EST-based oligo-design for our microarray platform (the chip was designed before the advent of the completed tomato genome; (The Tomato Genome Sequencing, 2012). Consequently, the microarray comprised a subset of the full transcriptome. Despite the fact that we analyzed only a subset of the full transcriptome, we were able to identify differentially expressed genes from sensitive and tolerant genotypes, possibly involved in the response to heat. The statistical analysis of the data from the microarray experiments as described in Chapter 4 was done using a combination of paired and unpaired significance analysis of microarray data (after normalization) that constitute a high stringency cut-off as opposed to the frequently used $\log 2$ fold cut off. This gives a relatively high confidence level for determining the significance of differentially expressed genes. Further analysis with Euclidian clustering and O2PLS analysis allowed the determination of probe sets showing differential expression that could be classified into GO categories.

The putative functions associated with the genes identified by cDNA-AFLP and microarrays indicate involvement of heat shock, metabolism, antioxidant and development pathways. Based upon the observed differences in response to MHS and screening of literature sources (Zinn et al., 2010) we identified a number of candidate transcripts involved in heat-tolerance. There is a good correspondence of gene expression modulation between the two systems (AFLP and microarrays) as was verified by quantitative PCR analysis (Chapters 3 and 4) and the major classes of genes found to be differentially expressed during MHS showed good agreement with other contemporary published studies (Frank et al., 2009).

The two major and novel results from our work indicate that firstly, tolerant lines respond more moderately to MHS and that secondly, a large number of genes that are differentially expressed after heat treatment in the sensitive line are already expressed in the tolerant genotype. The conclusion from these results is that it may be equally important to screen for constitutive differential gene expression (prior to the application of abiotic stress) as it is to search for key genes that are induced as a response to the stress. Although a clear identification of candidate genes responsible for heat tolerance could not easily be determined, a gene expression profile for tolerance could potentially be identified that would allow selection of tolerance prior to exposure to heat stress. Recently, a genomewide transcriptome analysis during anthesis revealed similar molecular responses to our tomato analysis in tolerant and sensitive rice varieties. The study also concluded that the appropriate expression of protective chaperones in anthers is needed to overcome stress damages and to ensure fertilization. Several transcripts among which HEAT SHOCK PROTEIN (HSP)101 and ROTAMASE FK506 ROF) 1 showed similar expression patterns following heat stress in both tomato and rice anthers (González-Schain et al., 2015; Li et al., 2015).

\section{Molecular aspects of tolerance to heat}

As opposed to other solanaceous species, tomato shows a pronounced sensitivity to high temperature during seed set, such that no fruit set occurs at day/night temperatures of $35^{\circ} / 23^{\circ} \mathrm{C}$. In our initial studies (Chapter 3), we used a standard commercial genotype (MM) that can be regarded as relatively sensitive to MHS. However, within the tomato germplasm there is a continuum of sensitivity and tolerance to high temperature. For a practical relevance of the microarray study (Chapter 4), two commercial hybrids were chosen, FR and HS1, which had been empirically determined to be sensitive and tolerant (respectively) by practical breeders. To augment this selection a wider set of genotypes was added to the analysis that showed various degrees of tolerance and sensitivity to high temperature, based on qPCR and pollen viability analyses (Chapter 5). In general, there was a good correspondence of the data from the different genotypes in relation with their heat sensitivity/tolerance phenotype. It is however, important to note that there is no absolute criterion for determining whether a genotype is 
tolerant or sensitive, and furthermore, sensitivity and tolerance are likely to interact strongly with other environmental factors such as soil type, water availability or nutrient status.

As heat tolerance is a multigenic character, numerous additional biochemical and metabolic processes are also involved in the development and maintenance of thermotolerance. Tolerant plants generally protect themselves from the damaging effects of heat stress with the synthesis of various antioxidant systems, protective osmolytes, secondary metabolites or phytohormones such as ABA. HSPs are of primary importance in thermotolerance responses and act as molecular chaperones to prevent denaturation or aggregation of target proteins as well as facilitating protein refolding (Ahuja et al., 2010; Feder and Hofmann, 1999; Wang et al., 2004). Heat tolerant rice anthers reveal a higher accumulation of small HSPs (sHSPs) in heat stress conditions (Jagadish et al., 2010). Similarly, several tolerant grape genotypes show higher levels of HSP70 and genes related to metabolism and stress protection under elevated temperatures (Zhang et al., 2005).

A comparison of the effects of heat stress on a heat-tolerant and a heat-sensitive tomato cultivar showed that temperature stress affected mainly the development of pollen grains, where reduced viability was more pronounced in the heat-sensitive cultivar (Firon et al., 2006). Moreover, a comparative transcriptomic analysis between tolerant and sensitive tomato genotypes suggests that the difference in tolerance to heat stress is associated with higher heat shock protein production under stressed conditions and lesser transcriptome responses in the heat-tolerant tomato genotype. In this case, the authors hypothesise that the capacity for thermotolerance could be achieved by constitutive expression levels of such 'responsive' genes prior to heat stress exposure (Frank et al., 2009). Several genes have been thus identified in various tolerant plant cultivars or wild germplasm, which were shown to improve photosynthesis and protect reproductive development, such as HSP101, stable Rubisco isoforms, etc. However, many genes identified by anther transcript profiling under heat show no homology to known sequences and represent potential candidates for further study of the tolerance to heat (Frank et al., 2009; Zinn et al., 2010).

We applied a moderate heat stress of $32^{\circ} \mathrm{C} / 26^{\circ} \mathrm{C}$ to profile the anther transcriptome response to heat in tomato genotypes with contrasting response to MHS. Similar to other studies, the heattolerant genotype exhibits a higher accumulation of genes encoding HSPs and fewer transcriptional changes than the heat-sensitive genotype upon MHS. Moreover, the heat-tolerant genotype also shows a different constitutive gene expression make-up compared to the heat-sensitive one, indicating differences in genetic adaptation to increased temperatures. In the heat-tolerant genotype, the majority of changes in gene expression is represented by up-regulation, while in the heat-sensitive genotype there is a general trend to down-regulate gene expression, soon after MHS. Based upon the observed differences in response to MHS we identified a number of candidate transcripts involved in heattolerance. A comparison of transcription profiles obtained from heat-stressed and non-stressed tomato microspores and a functional classification of the annotated transcripts show that the majority of the genes are involved in stress response and transcription regulation, including HSPs and genes associated with oxidative stress response, while a minority comprises signal transduction, metabolism and transport-related sequences, and cell wall modification processes.

The heat-tolerant genotype not only reacted differently from the heat-sensitive during MHS in terms of gene number, but also exhibited a characteristic gene expression pattern, characterised by very high expression levels of protection and repair genes. The heat shock Hsp82 (TC170030) and the gene coding for mitochondrial small heat shock protein (msHsp) LeMtHSP (TC187014) are highly expressed in HS1 at time point $0 \mathrm{~h}$ prior to the MHS. Another set of genes are responsive only in the heat-sensitive genotype, among which the heat shock proteins Hsa32 (TC191544), Rof1 (TC187143) and ROC7 (TC175253) genes required for protein folding. The extent to which the oxido-reduction processes are affected by MHS in the heat-sensitive genotype becomes evident from the differential regulation of genes such as the carotenoid cleavage dioxygenase 1 (TC181718), a steroid alphareductase, (TC170471), a flavonol synthase (TC172800) and a flavoprotein (TC83140. The stress 
response of the heat-sensitive genotype can be seen from repression of transcripts similar to pepsin A (TC171322) and subtilisine (TC181342). Unlike the heat-sensitive genotype, the heat-tolerant genotype HS1 shows fewer genes significantly induced in the protection and repair group.

Many plant species rely on basal mechanisms for their response to heat. An analysis of Chenopodium album L. populations from contrasting thermal environments reveals lower heat shock protein levels and induced thermotolerance in plants growing at higher temperatures and interestingly an HSP accumulation that decreases with increasing fluctuations of the habitat temperatures (Barua et al, 2008). When compared to other sensitive genotype, a grape tolerant genotype showed higher heat shock gene expression levels under standard growth conditions (Zhang et al, 2005). We also found that basal gene expression levels in tomato anthers of a number of genes appeared significantly differentially expressed in the tolerant genotype compared to the sensitive genotype (Bita et al, 2011). It is thus possible that plants are more tolerant due to a constitutive and active over-expression of stress-related genes even under standard growth conditions (Taji et al, 2004). Hence, when selecting for tolerance in tomato anthers it could be advisable to select for increased basal instead of inducible thermotolerance in breeding for heat tolerant crops (Barua et al, 2008).

In conclusion, the heat-tolerant genotype HS1 reacts to MHS by enhancing the expression of heat shock proteins, oxido-reductive, transport and stress protective transcripts. In stark contrast, the heat-sensitive FR shows a more extensive, wider transcriptomic response characterized firstly by a complementary modulation pattern as compared to the heat-tolerant genotype; e.g. transport and signaling genes are down-regulated in the heat-sensitive and up-regulated in the heat-tolerant. Secondly, the stress response in the heat-sensitive genotype is characterized by an increased expression of a more diverse range of functional classes including transcription, photosynthesis, hormone related and protection genes which are increased, while transport and carbohydrate metabolism are reduced in expression. Thirdly, although the two genotypes involve similar functional classes in the response to heat, each genotype modulates "common" and "specific" genes. The genotype-specific modulated transcripts are of particular interest for the present work as their annotation may provide clues to putative mechanisms of heat tolerance.

\section{Abscisic acid and male sterility under heat stress}

In plants, abscisic acid (ABA) often functions as a mediator of key phase-transitions throughout the plant life cycle and several lines of evidence indicate that basal levels of ABA, ABA synthesis, degradation and signalling exert a profound effect on plant growth, development and physiology (Arenas-Huertero et al., 2000; Cutler et al., 2010; Kotak et al., 2007), while under stress conditions including heat, ABA accumulation has been mostly assigned a negative function as stress signal, often being associated with male sterility (Saini \& Aspinall, 1982; Wilkinson \& Davies, 2010). Several data suggest that normal stamen development in tomato takes place in the presence of low ABA levels (Singh and Sawhney, 1998). Maintaining low ABA levels, at least in the reproductive tissues is therefore important for stress tolerance and pollen fertility. Both spermatogenesis and pollen mother cell development are inhibited by elevated temperature but the male generative cell development is more susceptible to heat stress. Although the mechanisms of how ABA interacts with the heat stress response in plants remains unclear, the early events in pollen development and the abortion of pollen mother cell maturation are intimately linked to ABA (Razem et al., 2006). We were interested in investigating the role of $\mathrm{ABA}$ in the tolerance to heat of the tomato reproductive tissues due to the similarity in the phenotype between heat-induced sterility and a male sterile ABA mutant. Both the study of ABA application and the phenotypic characterisation of the ABA mutant $s l-2$ confirm this relationship (Chapter 5).

In studying the role of $\mathrm{ABA}$ in abiotic stress tolerance, the focus has mainly been on the vegetative parts of the plant. In this case, ABA accumulation generally results in improved tolerance (Peleg and 
Blumwald, 2011). However, at the reproductive stage, there appears to be a negative correlation between $\mathrm{ABA}$ levels and abiotic stress tolerance, where genes involved in $\mathrm{ABA}$ transport and homeostasis are repressed while genes involved in $\mathrm{ABA}$ biosynthesis are activated. Cold-induced pollen sterility in cold-sensitive rice lines is aggravated by ABA accumulation in anthers (Oliver et al., 2007), and we show in Chapter 5 that there is a similar correlation for heat-induced sterility in tomato.

In our experiment performed on pairs of genotypes, heat stress induced abscisic acid (ABA) accumulation in anthers of heat-sensitive tomato genotypes. In contrast, the heat-tolerant genotypes accumulated lower ABA levels. However, there are additional levels of regulatory control (e.g. epigenetic, post-transcriptional, and post-translational) that may modify the abundance, localization or activity of enzymes involved in ABA metabolism. The high temperature extremes assessed in the current study might also dramatically alter pathways responsible for RNAi-mediated gene silencing (RdDM pathway) and genesis of small RNAs. Abiotic stress treatments and even the metabolic status of rapidly growing or inactive tissues also influence mRNA turnover and translation in plants (Baron et al., 2012). Currently, the role for ABA in reproductive development and abiotic stress tolerance of reproductive structures is ambiguous. Several studies propose that excessive ABA accumulation could have a negative impact on the abiotic stress tolerance of reproductive tissues (Ji et al., 2011).

Similar to our experiments, floret infertility in wheat was induced by the application of abscisic acid (ABA) directly to the developing spike over a 3-6-day period during pollen meiosis in several wheat cultivars. The major effect was failure of grain set in apparently competent florets. The wheat cultivars differed in both their sensitivity to ABA and in the yield components affected. A high temperature treatment $\left(27^{\circ} \mathrm{C}\right)$ for 7 days over the period of meiosis also reduced grain set and in some cultivars florets were lost. The cultivar most sensitive to high temperature was also most sensitive to $\mathrm{ABA}$. This result indicated a direct action of $\mathrm{ABA}$ in the spike and a possible indirect effect on assimilate supply (Zeng et al., 1985).

In our experiments, the heat sensitive genotype was too rendered sterile by ABA application as revealed by pollen germination tests and the ulterior lack of fruit set, and presented various degrees of anther malformations as a consequence of the extended heat treatment. In addition, regular ABA application on meiotic and younger flower buds of the heat sensitive genotype during maturation to open flowers resulted in a similar phenotype with that of the s 12 mutant. Interestingly, the heat tolerant genotype maintained its fertility and phenotype following ABA application. Although gene expression changes occur very rapidly after the imposition of heat stress, other effects including phenotypic changes require longer periods of heat stress and in particular at critical stages of development. The accumulation of ABA due to heat stress which is likely to be one of the reasons for a anther malformation is likely to require an extended period of heat stress (several days). However, this has not been systematically investigated in this thesis.

ABA accumulation under stressful conditions interrupts sugar transport to developing pollen grains by impairing sucrose hydrolysis, in rice. In fact, it was shown that ABA controls anther sink strength and abortion of pollen development under drought conditions in wheat (Ji et al., 2011),(Oliver et al., 2007). The subsequent lack of sucrose biosynthesis may be the most upstream molecular dysfunction leading to altered carbohydrate metabolism and starch deficiency under elevated growth temperature conditions (Jain et al., 2010) probably also by affecting the tapetum, as shown by the in situ hybridisation of all heat responsive genes to the tapetum results described in Chapter 3. Heat stress and $\mathrm{ABA}$ application resulted in premature tapetal degeneration followed by periplasmodial invasion of the locule at meiosis and total sterility in wheat (Saini, 1984).

In rice, cold treatment of a cold-sensitive cultivar resulted in increased anther ABA levels while exogenous $\mathrm{ABA}$ treatment at the young microspore stage induced pollen sterility and affected cell wall invertase and monosaccharide transporter gene expression in a way similar to the cold treatment. However, in a cold-tolerant cultivar, ABA levels were significantly lower under normal circumstances and remained low after cold treatment. The differences in endogenous ABA levels in 
the cold-sensitive and cold-tolerant cultivars correlated with differences in expression of the ABA biosynthetic genes encoding zeaxanthin epoxidase (OsZEP1) and 9-cis-epoxycarotenoid dioxygenase (OSNCED2, OsNCED3) in anthers. More, the expression of three ABA-8-hydroxylase genes $(A B A 8 O X 1,2$ and 3$)$ in the anthers of the tolerant cultivar was higher under control conditions and was regulated differently by cold compared with the sensitive genotype. These differences suggest that there is a strong association between ABA metabolism and the cold tolerance phenotype (Oliver et al., 2007). Our results show similar results for heat stress, in terms of heat tolerance and ABA accumulation. In our experiments, the general trend displayed by the stressed genotypes is to accumulate lower relative levels of $\mathrm{ABA}$ at the end of the experimental period compared to the initial stages in more heat tolerant genotypes, and of higher levels in the heat sensitive genotypes. We conclude from these results that the morphological changes in the floral tissues, the reduced pollen germination rate and the overall changes in ABA levels are correlated with the increased temperature. Whether these correlations are causally related is not clear. Furthermore, the variation in the initial levels of $\mathrm{ABA}$ in the tolerant and sensitive genotypes are inconsistent, with a very high initial concentration of $\mathrm{ABA}$ in the most tolerant genotype (Saladette) compared to a relatively low initial level in N5024 which is also tolerant to heat stress. It appears thus that there is a weak correlation between sensitivity to heat and changes in the ABA accumulation pattern. This may indicate a different mechanism of ABA turnover in these genotypes and suggests that the absolute ABA levels after two weeks of heat treatment are more informative, while constitutive ABA levels might not be relevant for tolerance to heat. Clearly more research is needed to resolve these issues.

\section{Conclusions and practical implications}

Temperature stress has devastating effects on plant growth and metabolism, as these processes have optimum temperature limits in every plant species. Global climate change makes high temperature a critical factor for plant growth and productivity. High temperature is now considered to be one of the major abiotic stresses for restricting crop production (Hasanuzzaman et al., 2012). In this thesis we examined the response to heat in tolerant and sensitive tomato genotypes by transcriptomic profiling of their male reproductive structures, the anthers. Our results are similar to those of previous studies that associate tolerance with increased molecular chaperone production such as HSP, antioxidant and osmolytes and a lower transcriptomic response in the heat tolerant genotypes. Our results also confirm the hypothesis that selection for tolerance might go towards constitutive expression of tolerance genes, especially in the view of recent heat waves during hot summer all over the planet. The several tolerant genotypes we tested showed in general higher accumulation of HSP and protective molecules during heat stress but also presented higher constitutive gene expression for some of these protectants. Comparative transcriptome analyses of tolerant vs sensitive plants represent an attractive alternative to classical tolerance detection studies in detecting the global genetic make-up of a plant that makes it tolerant to stress. However, the microarray technology has several limitations such as the background hybridization that limits the accuracy of expression measurements, particularly for transcripts present in low abundance. More, the probes differ considerably in their hybridization properties and the arrays are limited to interrogating only those genes for which probes are designed.

Unlike microarray technologies, RNA-Seq is the next-generation transcriptome analysis instrument that allows direct sequencing of transcripts by high-throughput sequencing tools and has considerable advantages for examining transcriptome fine structure such as the detection of novel transcripts, allele-specific expression and alternative splicing variants. RNA-Seq does not depend on genome annotation for prior probe selection and avoids the related biases introduced during hybridization of microarrays (Mutz et al., 2013).

Understanding the relationship between the responses to heat stress applied in a controlled environment chamber and the responses to heat under field conditions can be problematic. Although 
the temperature, light and humidity can be regulated, it is likely that growth chamber conditions do not reflect natural conditions in the field. The fluctuations in light conditions and associated temperature fluctuations as well as the airflow that the plant experiences under field conditions cannot be faithfully replicated under laboratory conditions. Furthermore, heat stress is rarely the only abiotic stress suffered by plants under natural conditions. The most closely associated stress with heat stress is drought stress. In order to isolate the effect of high temperature in our experiments, we have not exposed the plants to low water levels that commonly accompany high temperature stress. Despite being a valid approach that has also been taken by other authors (Frank et al., 2009), it will be important to analyze the effects of combined stress to achieve a better understanding of plant responses. A combination of drought and heat stress was found to alter plant metabolism in a novel manner compared with single stresses (Rizhsky et al. 2004).

Farmers and breeders have long known that it is often the simultaneous occurrence of several abiotic stresses, rather than a particular stress condition, that is most lethal to field crops. Recent studies (Nakashima et al., 2014), (Rivero et al., 2014)have revealed that the molecular and metabolic responses of plants to a combination of two different abiotic stresses is unique and cannot be directly extrapolated from the response of plants to each of the different stresses individually. Effective breeding for stress tolerance requires efficient screening procedures, identification of key traits in diverse donor or tolerant lines and understanding their inheritance and molecular genetics. Pollen viability and seed-set as measures of reproductive success under heat stress are also candidate traits for selection in breeding programs, and generally there is a strong correlation between pollen production and viability, anther dehiscence, and seed-set. Regardless the screening method however, a key objective for plant breeders is to develop an effective set of thermotolerance markers which can be used to further implement heat tolerance into crop species. The development of tomato lines with a heat tolerance similar to that of the donor parent can be achieved by using a combination of phenotypic and marker-assisted selection in each generation. The tolerant parent could be simultaneously or sequentially incorporated into commercial cultivars of tomatoes through breeding practices that apply simultaneous selection for heat-tolerance genotypes using molecular markers (Lin et al., 2006).

HSPs assist in protein folding and limit protein aggregation in multiple cellular compartments. Therefore, introgression of a gene by conventional or modern breeding is usually not sufficient to develop heat-tolerant lines, unless there is a large effect on a particular key process. In addition, achieving highest genetic gain via classical methods requires a just choice for the appropriate breeding strategy. A current approach to the challenge of high temperature tolerance is 'physiological' or 'developmental' trait breeding through recurrent selection using crop germplasm from regions with hot growing seasons (Cossani and Reynolds, 2012). In both cases, the targets are loci with high heritability that sustain yield at normal and elevated temperatures. The selection can be on combined heat and drought tolerance, as required by many crops. Recurrent selection has been successfully used to improve heat tolerance in wheat (Machado J.C, 2009) and potato (Benites and Pinto, 2011). Although stress adaptation traits that have evolved naturally can be transferred relatively fast in the field via introgression into established germplasm, transgenes are expressed constitutively or in an appropriate spatio-temporal manner.

The modification of major regulatory genes through biotechnology is considered to be more efficient than conventional breeding through serial hybridizations and linking several beneficial genes (transgenic pyramiding) into a commercial variety via the transgenic approach is also likely to provide a key route to crop improvement. In order to meet the future requirements of agricultural production, a proper assessment of environmental stress tolerance for crops needs thus to be designed and implemented. Multiple opportunities for improvement exist, as tolerance to high temperatures is a multigenic character involving a complex network of chaperones and other protective proteins acting together to defend the cells from heat injury. 
Abiotic stresses affect plants in a complex manner depending on the growth stage. The conventional and molecular plant breeding efforts are limited by the present lack of accurate phenotyping for stress responses, due to the incomplete understanding of tolerance mechanisms. Developing countries in particular, are still lacking in appropriate phenotyping facilities in the public research sector. In addition, generating high yielding and stress tolerant crops requires a thorough understanding of the metabolic and developmental processes involved, not only in stress responses, but also in energy regulation (Hirayama and Shinozaki, 2010). Such strategies are likely to improve stress tolerance but nonetheless, there is a lag from basic research to the production of stress-tolerant crops. Ultimately, differences in their research strategies and their primary concerns cause molecular biologists, plant physiologists and breeders to work independently, where an intimate collaboration amongst them is required. In conclusion, in order to meet the future requirements of agricultural production, a proper assessment of environmental stress tolerance for crops needs thus to be designed and implemented, including the field assessment of yield, which is an essential step in releasing commercial cultivars to farmers. Multiple opportunities for improvement exist, as tolerance to high temperatures is a multigenic character involving a complex network of chaperones and other protective proteins that defend the cells from heat injury. Well-funded, long-term, multinational, multidisciplinary collaborations are vital to making significant progress in developing new crop varieties to enhance food security, especially in developing countries.

There is a differential effect of climate change both in terms of geographic location and the crops that will likely show the most extreme reductions in yield, as a result of expected extreme fluctuations in temperature and global warming in general. At present, most of the Earth's arable land is already in production and what remains is being lost to urbanization, desertification or environmental degradation. The development and deployment of high-yielding crop varieties will make a vital future contribution to sustainable agriculture. Stress tolerant crops would also help improve land use by increasing yields on existing lands, reducing crop losses, and expansion onto new lands previously unproductive due to high levels of abiotic stress. 


\section{References}

Ahuja, I., de Vos, R.C.H., Bones, A.M. and Hall, R.D. (2010) Plant molecular stress responses face climate change. Trends in plant science 15, 664-674.

Arenas-Huertero, F., Arroyo, A., Zhou, L., Sheen, J. and Leon, P. (2000) Analysis of Arabidopsis glucose insensitive mutants, gin 5 and gin6, reveals a central role of the plant hormone ABA in the regulation of plant vegetative development by sugar. Genes \& development 14, 2085-2096.

Bachem, C.W.B., Vriezen, W.H., Bita, C.E. and del Carmen, A.F. (2008) AFLP-Based RNA Fingerprinting: Novel Variants and Applications. In: The Handbook of Plant Functional Genomics pp. 21-35. Wiley-VCH Verlag GmbH \& Co. KGaA.

Barnabás, B., Jäger, K. and Fehér, A. (2008) The effect of drought and heat stress on reproductive processes in cereals. Plant, cell \& environment 31, 11-38.

Baron, K.N., Schroeder, D.F. and Stasolla, C. (2012) Transcriptional response of abscisic acid (ABA) metabolism and transport to cold and heat stress applied at the reproductive stage of development in $<\mathrm{i}>$ Arabidopsis thaliana $</ \mathrm{i}>$. Plant Science 188, 48-59.

Benites, F.R.G. and Pinto, C.A.B.P. (2011) Genetic gains for heat tolerance in potato in three cycles of recurrent selection. Crop Breeding and Applied Biotechnology 11, 133-140.

Bita, C.E., Zenoni, S., Vriezen, W.H., Mariani, C., Pezzotti, M. and Gerats, T. (2011) Temperature stress differentially modulates transcription in meiotic anthers of heat-tolerant and heatsensitive tomato plants. BMC genomics 12, 384.

Bray, B.-S.W. (2000) Responses to abiotic stresses. Rockville, MD:American Society of Plant Physiologists.

Christensen, J.H. and Christensen, O.B. (2007) A summary of the PRUDENCE model projections of changes in European climate by the end of this century. Climatic Change 81, 7-30.

Cossani, C.M. and Reynolds, M.P. (2012) Physiological traits for improving heat tolerance in wheat. Plant physiology 160, 1710-1718.

Cutler, S.R., Rodriguez, P.L., Finkelstein, R.R. and Abrams, S.R. (2010) Abscisic acid: emergence of a core signaling network. Annual review of plant biology 61, 651-679.

Feder, M.E. and Hofmann, G.E. (1999) Heat-shock proteins, molecular chaperones, and the stress response: evolutionary and ecological physiology. Annual review of physiology 61, 243-282.

Firon, N., Shaked, R., Peet, M., Pharr, D., Zamski, E., Rosenfeld, K., Althan, L. and Pressman, E. (2006) Pollen grains of heat tolerant tomato cultivars retain higher carbohydrate concentration under heat stress conditions. Scientia Horticulturae 109, 212-217.

Frank, G., Pressman, E., Ophir, R., Althan, L., Shaked, R., Freedman, M., Shen, S. and Firon, N. (2009) Transcriptional profiling of maturing tomato (Solanum lycopersicum L.) microspores reveals the involvement of heat shock proteins, ROS scavengers, hormones, and sugars in the heat stress response. Journal of experimental botany 60, 3891-3908.

Grilli, G.V.G.B., L.T.; Lemos, E.G.M. (2007) QTL identification for tolerance to fruit set in tomato by AFLP markers. Crop Breed. Appl. Biotechnol. 7.

Grover, A., Mittal, D., Negi, M. and Lavania, D. (2013) Generating high temperature tolerant transgenic plants: Achievements and challenges. Plant science : an international journal of experimental plant biology 205-206, 38-47.

Guy, C. (1999) Molecular responses of plants to cold shock and cold acclimation. Journal of molecular microbiology and biotechnology 1, 231-242.

Hasanuzzaman, M., Hossain, M.A., da Silva, J.A.T. and Fujita, M. (2012) Plant response and tolerance to abiotic oxidative stress: antioxidant defense is a key factor. In: Crop Stress and its Management: Perspectives and Strategies pp. 261-315. Springer.

Hegarty, M.J., Jones, J.M., Wilson, I.D., Barker, G.L., Coghill, J.A., Sanchez-Baracaldo, P., Liu, G., Buggs, R.J., Abbott, R.J., Edwards, K.J. and Hiscock, S.J. (2005) Development of anonymous cDNA microarrays to study changes to the Senecio floral transcriptome during hybrid speciation. Molecular ecology 14, 2493-2510.

Hirayama, T. and Shinozaki, K. (2010) Research on plant abiotic stress responses in the post-genome era: past, present and future. The Plant Journal 61, 1041-1052.

Jagadish, S.V., Muthurajan, R., Oane, R., Wheeler, T.R., Heuer, S., Bennett, J. and Craufurd, P.Q. (2010) Physiological and proteomic approaches to address heat tolerance during anthesis in rice (Oryza sativa L.). Journal of experimental botany 61, 143-156. 
Jain, M., Chourey, P.S., Boote, K.J. and Allen, L.H., Jr. (2010) Short-term high temperature growth conditions during vegetative-to-reproductive phase transition irreversibly compromise cell wall invertase-mediated sucrose catalysis and microspore meiosis in grain sorghum (Sorghum bicolor). Journal of plant physiology 167, 578-582.

Ji, X., Dong, B., Shiran, B., Talbot, M.J., Edlington, J.E., Hughes, T., White, R.G., Gubler, F. and Dolferus, R. (2011) Control of abscisic acid catabolism and abscisic acid homeostasis is important for reproductive stage stress tolerance in cereals. Plant physiology 156, 647-662.

Kotak, S., Larkindale, J., Lee, U., von Koskull-Döring, P., Vierling, E. and Scharf, K.D. (2007) Complexity of the heat stress response in plants. Current opinion in plant biology 10, 310316.

Lin, K.-H., Yeh, W.-L., Chen, H.-M. and Lo, H.-F. (2010) Quantitative trait loci influencing fruitrelated characteristics of tomato grown in high-temperature conditions. Euphytica 174, 119135.

Lin, K.H., Lo, H.F., Lee, S.P., George Kuo, C., Chen, J.T. and Yeh, W.L. (2006) RAPD markers for the identification of yield traits in tomatoes under heat stress via bulked segregant analysis. Hereditas 143, 142-154.

Lohar, D. and Peat, W. (1998) Floral characteristics of heat-tolerant and heat-sensitive tomato $(<\mathrm{i}>$ Lycopersicon esculentum $</ i>$ Mill.) cultivars at high temperature. Scientia Horticulturae 73, 53-60.

Machado J.C, e.a. (2009) Recurrent selection as breeding strategy for heat tolerance in wheat. Crop Breeding and Applied Biotechnology $\mathbf{1 0 .}$

Maestri, E., Klueva, N., Perrotta, C., Gulli, M., Nguyen, H.T. and Marmiroli, N. (2002) Molecular genetics of heat tolerance and heat shock proteins in cereals. Plant molecular biology 48, 667681.

Mutz, K.-O., Heilkenbrinker, A., Lönne, M., Walter, J.-G. and Stahl, F. (2013) Transcriptome analysis using next-generation sequencing. Current opinion in biotechnology 24, 22-30.

Nakashima, K., Yamaguchi-Shinozaki, K. and Shinozaki, K. (2014) The transcriptional regulatory network in the drought response and its crosstalk in abiotic stress responses including drought, cold, and heat. Front Plant Sci 5, 170.

Oliver, S.N., Dennis, E.S. and Dolferus, R. (2007) ABA regulates apoplastic sugar transport and is a potential signal for cold-induced pollen sterility in rice. Plant and Cell physiology 48, 13191330.

Peet, M., Sato, S. and Gardner, R. (2002) Comparing heat stress effects on male-fertile and malesterile tomatoes. Plant, cell \& environment 21, 225-231.

Peleg, Z. and Blumwald, E. (2011) Hormone balance and abiotic stress tolerance in crop plants. Current opinion in plant biology 14, 290-295.

Razem, F.A., Baron, K. and Hill, R.D. (2006) Turning on gibberellin and abscisic acid signaling. Current opinion in plant biology 9, 454-459.

Rivero, R.M., Mestre, T.C., Mittler, R., Rubio, F., Garcia-Sanchez, F. and Martinez, V. (2014) The combined effect of salinity and heat reveals a specific physiological, biochemical and molecular response in tomato plants. Plant, cell \& environment 37, 1059-1073.

Saini, H.S., M. Sedgley, and D. Aspinall (1984) Developmental anatomy in wheat of male-sterility induced by heat-stress, water deficit or abscisic-acid. . Aust. J. Plant Physiol. 11

Sakata, T. and Higashitani, A. (2008) Male sterility accompanied with abnormal anther development in plants-genes and environmental stresses with special reference to high temperature injury. International Journal of Plant Developmental Biology 2, 42-51.

Sato, S., Kamiyama, M., Iwata, T., Makita, N., Furukawa, H. and Ikeda, H. (2006) Moderate increase of mean daily temperature adversely affects fruit set of Lycopersicon esculentum by disrupting specific physiological processes in male reproductive development. Annals of botany 97, 731-738.

Singh, S. and Sawhney, V. (1998) Abscisic acid in a male sterile tomato mutant and its regulation by low temperature. Journal of experimental botany 49, 199-203.

Sumesh, K., Sharma-Natu, P. and Ghildiyal, M. (2008) Starch synthase activity and heat shock protein in relation to thermal tolerance of developing wheat grains. Biologia plantarum 52, 749-753. 
The Tomato Genome Sequencing, C. (2012) The tomato genome sequence provides insights into fleshy fruit evolution. Nature 485, 635-641.

Upadhyaya, H.D., Dronavalli, N., Gowda, C. and Singh, S. (2011) Identification and evaluation of chickpea germplasm for tolerance to heat stress. Crop Sci 51, 2079-2094.

Wang, X., Liu, L., Liu, S., Sun, X., Deng, Z., Pi, Y. and Tang, K. (2004) Isolation and molecular characterization of a new CRT binding factor gene from Capsella bursa-pastoris. Journal of biochemistry and molecular biology 37, 538-545.

Weis, E. and Berry, J.A. (1988) Plants and high temperature stress. Symposia of the Society for Experimental Biology 42, 329-346.

Young, L.W., Wilen, R.W. and Bonham-Smith, P.C. (2004) High temperature stress of Brassica napus during flowering reduces micro-and megagametophyte fertility, induces fruit abortion, and disrupts seed production. Journal of experimental botany 55, 485-495.

Zeng, Z., Morgan, J. and King, R. (1985) Regulation of grain number in wheat: Genotypic difference and responses to applied Abscisic acid and to high temperature. Functional Plant Biology 12, 609-619.

Zhang, J., Huang, W., Pan, Q. and Liu, Y. (2005) Improvement of chilling tolerance and accumulation of heat shock proteins in grape berries $(<\mathrm{i}>$ Vitis vinifera $</ \mathrm{i}>\mathrm{cv}$. Jingxiu) by heat pretreatment. Postharvest biology and technology 38, 80-90.

Zinn, K.E., Tunc-Ozdemir, M. and Harper, J.F. (2010) Temperature stress and plant sexual reproduction: uncovering the weakest links. Journal of experimental botany 61, 1959-1968. 


\section{Summary}

Global warming already has and will significantly impact crop productivity and yield in the near future. In order to meet the forecasted requirements of the future agricultural production, a proper assessment of crops environmental stress tolerance needs to be designed and implemented, from the laboratory to field. Genetic variation in the ability of tomatoes to set fruit under high temperature conditions has made selection for heat tolerance possible and multiple opportunities for improvement exist, as tolerance to high temperatures is a multi-genic character involving a complex network of chaperones and other protective proteins acting together to defend the cells from heat injury. Breeding programs involved in the development of heat tolerant cultivars should identify and make use of such tolerance traits already available in collected or wild germplasm.

The goal of this thesis was to characterize the response to high temperatures in meiotic tomato anthers with contrasting responses to heat and to identify genes that could be related to thermotolerance mechanisms during gamete development. Several molecular tools such as transcriptomic profiling by cDNA-AFLP and microarray analysis, RT-PCR or in situ RNA hybridisation were used to achieve this goal.

The second chapter reviews the effects of heat stress on reproductive flower development, candidate tolerance pathways and methods for production of heat tolerant crops.

The third chapter provides a general overview of the expression changes occurring in the developing anthers of a sensitive tomato genotype following exposure to a (short and) moderate high temperature stress (MHS). Using a combination of cDNA-AFLP, RT-PCR, and in situ RNA hybridisation, we characterized and verified the general transcriptional response to heat of tomato plants. Our results revealed that approximately $1 \%$ of the examined transcript-derived fragments exhibit alterations in expression pattern and the majority of these were down-regulated The putative functions associated with the genes identified by cDNA-AFLP indicated involvement of heat shock, metabolism, antioxidant and developmental processes. Based upon the observed transcriptional changes in response to MHS and on literature sources, we identified a number of candidate transcripts to be involved in heat-tolerance. The spatial expression of several such candidate genes was further examined using in situ RNA hybridisation and this showed that the investigated genes are expressed in the tapetum or/and in developing microspores. Furthermore, the expression of several candidate genes has been quantified by RT-PCR in additional genotypes with different degrees of heat tolerance. The results suggested a correlation between gene expression levels, pollen germination rates and tolerance to heat (Chapter 4).

In the fourth chapter we proceeded to profile the response to heat of meiotic anthers in a tolerant and a sensitive tomato genotype and investigated the expression of the identified candidate genes in several pairs of contrasting genotypes. Using microarray analysis (for an extensive overview of the meiotic response to heat) and RT-PCR, we were able to clearly distinguish differential responses of the tolerant genotype. After $2 \mathrm{~h}$ of moderate heat stress, the heat-tolerant genotype exhibits fewer transcriptional changes than the heat-sensitive genotype. In the heat-tolerant genotype, the majority of changes in gene expression is represented by up-regulation, while in the heat-sensitive genotype there is a general trend to down-regulate gene expression soon after MHS. Moreover, the heat-tolerant genotype also shows a different level of constitutive gene expression profiles when compared to the heat-sensitive genotype indicating a difference in genetic adaptation with regards to increased temperatures. The putative functions associated with the genes identified by microarray profiling indicate involvement of heat shock, antioxidant, metabolic, and cell development pathways. Based upon the observed differences in response to MHS we selected a number of candidate transcripts involved in heat-tolerance and confirmed their expression pattern in different tomato genotypes with contrasting responses to heat. The results suggested that the candidate genes are involved in the activation of protection mechanisms in the tomato anthers during moderate heat stress 
and, could therefore contribute to normal growth and development of the male gametophyte and implicitly a successful fruit set under adverse temperatures.

In the fifth chapter we tested the hypothesis that heat tolerance is associated with maintenance of organ identity, fertility and lower ABA levels during heat stress (for several tomato genotypes) and analysed the dynamics of ABA accumulation under temperature stress in several tomato genotypes with contrasting responses to heat. Furthermore, pollen germination tests were performed and additional physiological aspects of anther development for each genotype were analysed as well. The general trend observed was the accumulation of lower relative levels of ABA at the end of the experimental period compared to the initial stages in more tolerant genotypes and of higher levels in the sensitive genotypes. We concluded from these results that the morphological changes in the floral tissues and the overall changes in ABA levels are correlated with the molecular responses under increased temperature in the genotypes analysed. Whether these correlations are causally related is not clear; therefore more research is needed to resolve these issues.

The sixth chapter examines our analysis of the heat stress response in meiotic tomato anthers in a broader scientific context. I discuss the different aspects of our results and present several candidate genes involved in plant thermo-tolerance. In addition, I also discuss the potential involvement of plant growth regulators in plants' responses to heat stress and suggest various potential follow-up experimental strategies. 


\section{Sanmenvatting}

Het opwarmen van de aarde heeft en zal een groot effect hebben op de opbrengst van gewassen in de nabije toekomst. Om aan de verwachte toekomstige landbouwproductie te kunnen voldoen is het essentieel, om strategieën te ontwikkelen die planten resistent maken tegen abiotische stress en deze te implementeren in de praktijk. Bij tomaat bestaat er genetische variatie in de eigenschap die vruchtvorming bij hogere temperaturen toestaat. Dit heeft het mogelijk gemaakt om genen te selecteren die bijdragen aan hitte tolerantie. Dit resulteert in verschillende mogelijkheden om tomaten rassen te verbeteren, omdat het een eigenschap is die door meerdere genen gereguleerd wordt. Het betreft een complex netwerk van chaperonen en andere helper eiwitten die gezamenlijk de cel beschermen tegen hitte beschadiging. Toekomstige veredelingsprogramma's die er op gericht zijn om hitte tolerante tomaten te maken, zullen hitte tolerantie eigenschappen die voorkomen in bestaande collecties, kunnen gebruiken,

Het doel van dit proefschrift was de karakterisering van de respons van meiotische helmhokjes op een hitte behandeling. Hierbij zijn tomaten gebruikt met verschillende responsen. Deze zijn gebruikt om genen to identificeren die een rol kunnen spelen in mechanismen die hitte tolerantie tijdens de vorming van gameten reguleren. Hiervoor zijn een set van moleculaire methoden gebruikt, zoals; karakterisering van de mRNA populatie, cDNA-AFLP, microarray analyse, RT-PCR en in situ hybridisatie.

Het tweede hoofdstuk geeft een overzicht van de effecten van hitte stres op bloemontwikkeling in het bijzonder de aspecten die voor de voortplanting van betekenis zijn. Verder is beschreven wat bekent is van hitte tolerantie mechanismen en welke methoden er gebruikt worden om hitte tolerante tomaten te maken.

Het derde hoofdstuk geeft een overzicht van de genexpressie veranderingen in helmhokjes van een hitte gevoelige tomaat die aan hitte stress wort blootgesteld. Dit liet zien dat de expressie van ongeveer $1 \%$ van de genen is veranderd. In de meeste gevallen komen deze gene lager tot expressie. De genen met het veranderde expressie niveau coderen voor eiwitten betrokken bij hitte stress metabolisme, ontwikkelingsprocessen of antioxidant functies hebben. Binnen deze groep van genen met een veranderde expressie hebben wij een aantal kandidaat genen geselecteerd en van deze hebben we m.b.v. in situ hybridisatie bestudeert waar en wanneer deze gene tot expressie komen. Dit liet zien dat ze tot expressie komen in tapetum of in de zich ontwikkelende microsporen. Verder is de expressie van deze gene met RT-PCR gekwantificeerd in tomaten lijnen met verschillende niveaus van hitte tolerantie en dat liet zien dat er mogelijk een correlatie is tussen expressie niveau en hitte tolerantie en snelheid waarmee pollen kiemen.

In hoofdstuk 4 hebben we de responsen van hitte tolerante en gevoelige tomaten genotype paren met elkaar vergeleken. Hierbij werd gekeken naar de in hoofdstuk 3 geselecteerd kandidaat genen en m.b.v. microarray studies is een overzicht van de expressie verschillen verkregen. Hiermee waren we in staat differentiële responsen die karakteristiek zijn voor tolerante tomaat genotypen te identificeren. Na 2uur blootstelling aan hitte stres is de verandering in genexpressie in de tolerante lijnenbeduidend minder dan in de gevoelige lijnen. Verder betreffen de genexpressie verandering in de tolerante lijnen vooral een verhoogde expressie, terwijl in de gevoelige lijnen dit meestal verlaagd is. Verder is het profiel van de constitutief tot expressie komende genen in tolerante en gevoelige genotypen verschillend, wat suggereert dat deze tolerante lijnen genetisch anders geprepareerd zijn voor een hitte stres. De mogelijke functies van de genen die met micro-array analyse zijn geïdentificeerd suggereert dat ze coderen voor eiwitten betrokken bij hitte stress metabolisme, ontwikkelingsprocessen of antioxidant functies hebben. We hebben binnen deze groep van genen kandidaten geselecteerd die betrokken kunnen zijn bij hitte tolerantie. De expressie patronen werden goed gekwantificeerd in verschillende paren van tolerante en gevoelige lijnen. Dit liet zien dat deze 
gene kunnen bij dragen aan hitte tolerantie en het mogelijk maken dat tomatenvruchten gevormd worden bij hoge temperaturen.

In hoofdstuk 5 hebben we de hypothese getoetst dat hitte tolerantie gekoppeld is met behoud van orgaan identiteit, vruchtbaarheid en lagere ABA niveaus. We hebben de dynamiek van ABA accumulatie gedurende hitte stres in gevoelige en tolerante tomaat genotype bestudeerd. Verder hebben we pollen kieming bestudeerd. Dit laat zien dat gedurende ontwikkeling de ABA niveaus afnemen in de tolerante lijnen, terwijl dit in de gevoelige lijnen toeneemt. Er is dus een correlatie tussen hitte geïnduceerde veranderingen in genexpressie, en morfologische verandering in bloemweefsels en veranderingen in ABA niveaus. Of deze correlaties causaal van aard zijn zal in de toekomst bestudeerd moeten worden.

In hoofdstuk 6 worden de behaalde resultaten in een bredere context geplaatst. Ik bediscussieer verschillende aspecten van onze resultaten o.a. welke geselecteerde kandidaat genen de beste hitte tolerantie zouden kunnen bewerkstelligen, de rol van planten groeifactoren in dit proces. Uiteindelijk bediscussieer ik strategieën voor toekomstig onderzoek. 


\section{Curriculum Vitae}

Craita Elena Bita was born in Bucharest (Romania) in 1978, graduated in Plant Biotechnologies at USAMV Bucharest in 2001 and continued with a Master Degree in Horticultural Biotechnologies at MAICh in Greece in 2003. Later on she took on postgraduate research projects at several laboratories in Germany, the Netherlands and Greece, using molecular markers and transcriptomics tools to investigate the molecular response of environmental effects on plant growth and reproductive development. She started her PhD project in 2006 on fingerprinting the heat stress effects during tomato anther development. During this period, she also was involved in additional activities such as membership of the EPS PhD Student Council, organization of several scientific events, acting as reviewer for several scientific journals and as guest editor for a special edition of Frontiers of Pant Sciences, Nature Publishing Group. 


\section{Acknowledgments}

I would like to thank to all people who made this experience possible and helped thus contributing to my personal and professional development. It all started with the ADONIS project, an international effort focused on revealing molecular aspects of tolerance to abiotic stress in plants, project funded by the Marie Curie Research Fellowship Programme.

Grateful thanks to, Gerco Angenent, Christian Bachem and Tom Gerats for their guidance, motivation and support during the preparation of my thesis. I am also very grateful to Ton Bisseling and the EPS team for facilitating the transfer of the $\mathrm{PhD}$ to Wageningen-UR. Valuable suggestions and critical comments from Wim Vriezen and Titi Mariani helped improve my research experience. Special thanks go to Sara Zenoni and Mario Pezzotti from Verona for their help in analysing the microarray data and to Uwe Druege and Tatsiana Charnikhova for performing ABA extraction and detection in our heat stressed samples. Our secretary Else - very special thanks and appreciation for the help given, our lab technicians Mieke and Peter, the greenhouse people Ivette, Harry and Walter, Gerard, and all my former colleagues Maaike, Marian, Thikra, Lisette, Janny, Klaas, Antoin, Jan, Mena, Anneke, Veena, Anna, Tomek, Tati, and so many others I have met and received helpful advice from, during my studies in the Netherlands. 


\section{Education Statement of the Graduate School \\ Experimental Plant Sciences}

Issued to:

Date:

Craita Elena Bita

Group:

7 June 2016

University:

Laboratories of Plant Breeding and Molecular Biology

Wageningen University \& Research

\section{1) Start-up phase}

- First presentation of your project

Tomato flower development under heat stress conditions

- Writing or rewriting a project proposal

Abscisic acid (ABA) specific transcriptome profiling in heat-stressed tomato reproductive organs

- Writing a review or book chapter

AFLP-Based RNA Fingerprinting: Novel Variants and Applications , in The Handbook of Plant Functional

Genomics: Concept and Protocols, Wiley. DOI: 10.1002/9783527622542.ch2

Plant tolerance to high temperature in a changing environment: scientific fundamentals and production of heat

stress-tolerant crops, Frontiers in Plant Science (2013), Vol 4. DOI: 10.3389/fpls.2013.00273

MSc courses

- Laboratory use of isotopes

Course Radiation Protection Proficiency Level 5B

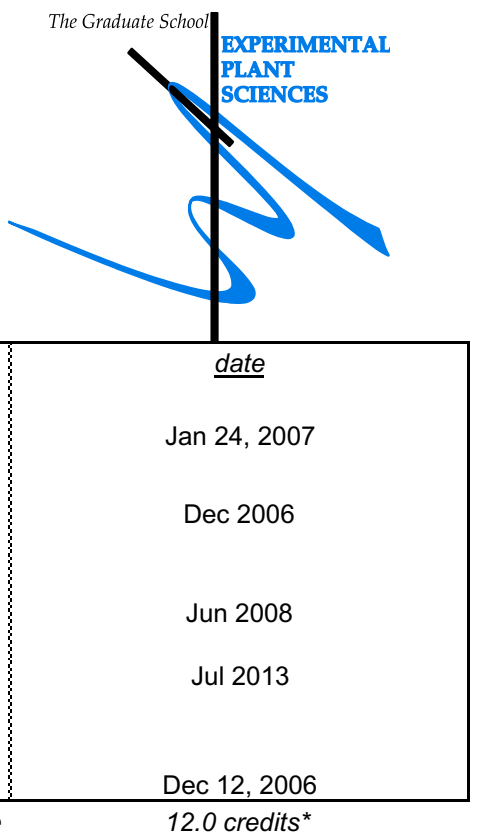

Subtotal Start-up Phase

\section{$\underline{\text { date }}$}

\section{2) Scientific Exposure}

\section{- EPS PhD student days}

EPS PhD student day 2007, Wageningen University

EPS PhD student day 2009, Leiden University

Sep 13, 2007

EPS theme symposia

EPS Theme 4 Symposium 'Genome Plasticity', Radboud University Nijmegen

EPS Theme 4 Symposium 'Genome Plasticity', Leiden University

EPS Theme 4 Symposium 'Genome Plasticity', Wageningen University

EPS Theme 4 Symposium 'Genome Plasticity', Radboud University Nijmegen

Feb 26, 2009

Lunteren days and other National Platforms

Annual Meeting 'Experimental Plant Sciences', Lunteren (NL)

Annual Meeting 'Experimental Plant Sciences', Lunteren (NL)

Annual Meeting 'Experimental Plant Sciences', Lunteren (NL)

Annual Meeting 'Experimental Plant Sciences', Lunteren (NL)

Dec 08, 2006

Dec 07, 2007

Dec 12, 2008

Dec 11, 2009

- Seminars (series), workshops and symposia

Seminar series IWWR

ADONIS RNAomics minisymposium

Apr 02-03, 2007

Apr 07-08, 2008

Apr 05-06, 2009

Apr 19-20, 2010

\section{Seminar plus}

2006-2010

Aug 07, 2008

- International symposia and congresses

EMBO Conference Series on Plant Molecular Biology 'From basic genomics to systems biology', Ghent,

Belgium

1st European Retreat of PhD students in Experimental Plant Sciences, Wageningen, NL

SOL 2008, Cologne Germany

SOL 2012, Neuchatel, Switzerland

- Presentations

1st European PhD Retreat (Poster)

SOL 2008 (Poster)

SOL 2012 (Talk)

IAB interview

Meeting with a member of the International Advisory Board of EPS

May 02-04, 2007

Oct 02-03, 2008

Oct 13-16, 2008

Aug 26-30, 2012

Oct 02-03, 2008

Oct 13-16, 2008

Aug 26-30, 2012

- Excursions

Dec 05, 2008 
- EPS courses or other PhD courses

Gateway technology course, Wageningen, NL

Functional protein analysis, ADONIS course, Cologne, Germany

In vivo imaging and proteome analysis, ADONIS course, Munich, Germany

Proteomics, ADONIS course, Freising, Germany

Microscopy, ADONIS course, Nijmegen, Netherlands

Abiotic stress, ADONIS course, Oeiras, Portugal

Nov 20-24, 2006
Mar 19-30, 2007

Sep 29-Oct 5, 2007

Mar 03-07, 2008

Aug 08, 2008

Journal club

Individual research training

Mar 01-10, 2009

Subtotal In-Depth Studies

9.0 credits $^{*}$

\section{4) Personal development}

- Skill training courses

Scientific presentation: Presenting with confidence

Project and time management, Wageningen

Advanced Scientific writing

Getting Funded: Writing an Individual Fellowship Application

- Organisation of PhD students day, course or conference

ADONIS RNAomics minisymposium

1st Joint PhD Retreat of students in Plant Science

date

Membership of Board, Committee or PhD council

EPS PhD Council since 2007

\begin{tabular}{c|c} 
date \\
Sep 26-28, 2007 \\
Nov-Dec 2007 \\
Aug 04-06, 2008 \\
Mar 01-06, 2009
\end{tabular}

Aug 07, 2008

Oct 02-03, 2008

2007-2010

Subtotal Personal Development

7.6 credits* $^{*}$

\section{TOTAL NUMBER OF CREDIT POINTS*}

40,6

Herewith the Graduate School declares that the PhD candidate has complied with the educational requirements set by the Educational Committee of EPS which comprises of a minimum total of 30 ECTS credits

${ }^{*}$ A credit represents a normative study load of 28 hours of study. 